\title{
On beliefs and incomplete preferences in noncooperative games
}

Citation for published version (APA):

Schulteis, T. J. W. (2007). On beliefs and incomplete preferences in noncooperative games. [Doctoral Thesis, Maastricht University]. Universitaire Pers Maastricht. https://doi.org/10.26481/dis.20070607ts

Document status and date:

Published: 01/01/2007

DOI:

$10.26481 /$ dis.20070607ts

Document Version:

Publisher's PDF, also known as Version of record

\section{Please check the document version of this publication:}

- A submitted manuscript is the version of the article upon submission and before peer-review. There can be important differences between the submitted version and the official published version of record.

People interested in the research are advised to contact the author for the final version of the publication, or visit the DOI to the publisher's website.

- The final author version and the galley proof are versions of the publication after peer review.

- The final published version features the final layout of the paper including the volume, issue and page numbers.

Link to publication

\footnotetext{
General rights rights.

- You may freely distribute the URL identifying the publication in the public portal. please follow below link for the End User Agreement:

www.umlib.nl/taverne-license

Take down policy

If you believe that this document breaches copyright please contact us at:

repository@maastrichtuniversity.nl

providing details and we will investigate your claim.
}

Copyright and moral rights for the publications made accessible in the public portal are retained by the authors and/or other copyright owners and it is a condition of accessing publications that users recognise and abide by the legal requirements associated with these

- Users may download and print one copy of any publication from the public portal for the purpose of private study or research.

- You may not further distribute the material or use it for any profit-making activity or commercial gain

If the publication is distributed under the terms of Article $25 \mathrm{fa}$ of the Dutch Copyright Act, indicated by the "Taverne" license above, 
On beliefs and incomplete preferences in noncooperative games

Tim Jozef Wolfgang Schulteis 
ISBN 978-90-5278-640-7 


\title{
On beliefs and incomplete preferences in noncooperative games
}

\section{PROEFSCHRIFT}

\author{
ter verkrijging van de graad van doctor \\ aan de Universiteit Maastricht, \\ op gezag van de Rector Magnificus, \\ prof. mr. G.P.M.F. Mols \\ volgens het besluit van het College van Decanen, \\ in het openbaar te verdedigen op \\ donderdag 7 juni 2007 om 14.00 uur
}

door

Tim Jozef Wolfgang Schulteis

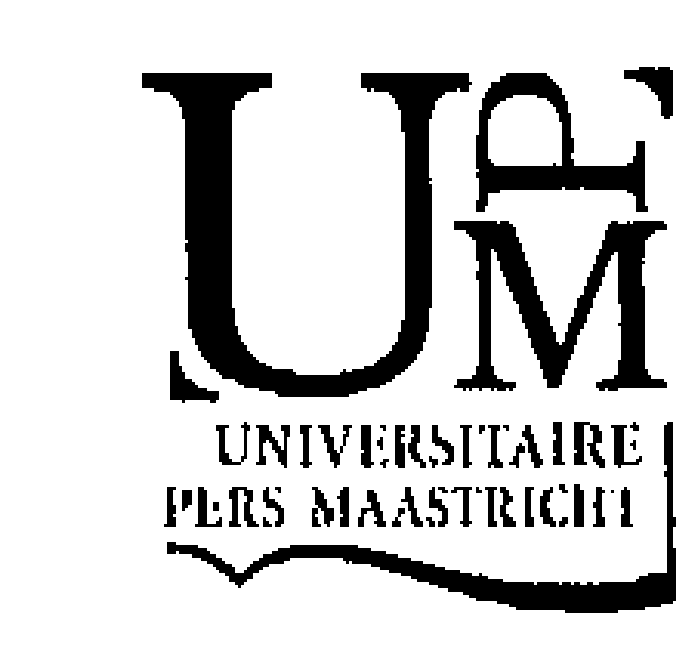




\section{Promotor:}

Prof. dr. Hans Peters

\section{Copromotoren:}

Dr. Andrés Perea

Dr. Dries Vermeulen

\section{Beoordelingscommissie:}

Prof. dr. Peter Wakker (voorzitter)

Prof. dr. Geir Asheim (University of Oslo, Norway)

Prof. dr. Jean-Jacques Herings 


\section{Contents}

1 Introduction 5

$\begin{array}{lll}\text { I Beliefs } & 15\end{array}$

2 Utility revision in signaling games $\quad 17$

2.1 Preliminaries . . . . . . . . . . . . . . . . . 20

2.1 .1 Sequential equilibrium . . . . . . . . . . . . . . . . 21

2.1 .2 Preference conjecture equilibrium . . . . . . . . . . . 24

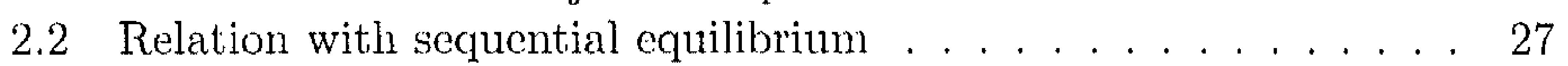

2.3 Minimum revision equilibrium . . . . . . . . . . . . . . . . 29

2.3.1 Minimum revision equilibrium . . . . . . . . . . 30

2.3.2 Relation with sequential equilibrium . . . . . . . 31

2.4 Examples and the intuitive criterion . . . . . . . . 36

2.5 Structure and computation . . . . . . . . . . . . 38

2.6 Remaining proofs . . . . . . . . . . . . . . . . 41

$\begin{array}{lll}3 & \text { Uncertainty about utilities } & 47\end{array}$

3.1 Introduction . . . . . . . . . . . . . . . . 47

3.2 Commonly known utilities . . . . . . . . . . . . . . . . . . . 48

3.2.1 The epistemic model . . . . . . . . . . . . . . . . . . 49

3.2 .2 Common belief in rationality . . . . . . . . . . . . . 49

3.2 .3 Dominated strategies . . . . . . . . . . . . . . . . 50

3.2 .4 Example . . . . . . . . . . . . . . . . . 51

3.3 Modelling uncertainty about utilities . . . . . . . . . . 52

3.3 .1 The epistemic model . . . . . . . . . . . . . . . . 52

3.3 .2 Common belief in rationality . . . . . . . . . . . 53

3.3.3 Dominated strategies. . . . . . . . . . . . . . . . . 53

3.3.4 An example . . . . . . . . . . . . . . . . . . . 54

3.4 Rationality and iterative elimination . . . . . . . . 55

3.4.1 Closedness under Harsanyi aggregation . . . . . . . . 55

3.4 .2 Main result . . . . . . . . . . . . . . . . . . 58

3.5 A special case . . . . . . . . . . . . . . 60

3.5.1 Cournot model of duopoly . . . . . . . . . . . . . . 60 
II Incomplete preferences $\quad 65$

4 Stochastic dominance equilibria $\quad 67$

4.1 Stochastic dominance . . . . . . . . . . . . . . . 68

4.2 Two-person games and t-equilibria . . . . . . . . . . . . . 71

4.3 An example ..................... . . . 72

4.4 Existence and characterization of $t$-equilibria . . . . . . . 74

4.5 Limit behavior of tequilibria . . . . . . . . . . . . . . . . . 76

4.6 Concluding remarks ................... 82

5 Correlated stochastic dominance $\quad 83$

5.1 Correlated equilibrium and correlated $t$-equilibria . . . . . . . 84

5.2 Existence of correlated $t$-equilibria . . . . . . . . . . . . 85

5.3 Sequences of correlated t-cquilibria . . . . . . . . . . 88

6 Generalized stochastic dominance $\quad 95$

6.1 Preliminaries . . . . . . . . . . . . . . . . . 996 96

6.2 Stochastic dominance . . . . . . . . . . . . . 96

6.3 Bad outcome aversion . . . . . . . . . . . . . . . . . . . . . . . . . . . . . . . . . 109

6.4 Game theoretic implications . . . . . . . . . . . . . . . . . . . 105

6.4 .1 Best replies and equilibria . . . . . . . . . . . 105

6.4 .2 Sequences of preference relations . . . . . . . . . . 107

$\begin{array}{lll}7 & \text { Samenvatting } & 117\end{array}$ 


\section{Preface}

This thesis is the result of my work as a Ph.D. student during the last five years. Of course it could not have be written without the help and support of many people. When I started my research, I also started my musical studies of singing and choral conducting, so first of all I would like to thank my promotor Hans Peters for giving mo the opportunity to work as a parttime Ph.D. student at the department of quantitative economics under these conditions. I also thank my (co-)promotores Andrés Perea, Dries Vermeulen and, again, Hans Peters for all the meetings we had to discuss the content of this thesis. Both this thesis as a whole and my general understanding of the field of game thcory were heavily influenced by these meetings, and by the very regular lunches, coffees and discussions with Andrés Perea.

But of course there is a lot more than mathematical skills only. Fully expressing the gratitude towards my parents for developing my human skills in the past 27 years by all their patience, understanding and care would go far beyond the approximately 120 pages of this thesis. Therefore, I will not even give it a try, nor will I try to exhaust the reader with expressing the feeling of luck that I found meeting Esther, who is now my wife, since I hope that everyone knows how necessary a general state of hapiness is for keeping the mind capable of solving mathematical problems. I admire Esther's capability of always asking the right questions, no matter if the topic is music, which is her own passion and profession, the things I try to explain to her about game theory or just life in general. Last, but not least, I express my thanks towards Ria and Jan for showing me that writing a dissertation in game theory and theoretically studying conjectures about the opponents' behaviour is of no good in many game theoretic experiments and playing cards in particular. 


\section{Chapter 1}

\section{Introduction}

This introduction is meant to give the interested reader who is not specifically skilled or trained in the field of game theory a very general idea of the topics that are dealt with in this dissertation. Readers who know more about the topic can find more cletailed information in the introductions to the chapters, since every chapter has its own introduction.

Game theory analyzes situations in which two or more decision makers interact in the sense that the final consequence, implying a utility for each decision maker, depends on each decision taken. The game is the set of data that defines the situation, such as the number of decision makers (players) and the rules that are to be followed, e.g. whether everyone reaches a decision sinultaneously or some players first observe decisions taken by opponents. In the literature a distinction is made between cooperative and noncooperative game theory. In a cooperative game players are supposed to work together (cooperate). The problem for the players that cooperate (form a coalition) is to fairly, with respect to some criterion they agreed upon, divide the joint utility among the players in the coalition. In this dissertation we will focus on noncooperative game theory, in which players are not assumed to form coalitions and players reach decisions by merely having the incentive of maximizing their individual utility. For some fixed player we will call every other decision maker an opponent.

We assume that players act optimally given their view on the situation (players are rational) and base their decisions on somc way of reasoning (leading to a belief) about the opponents' behaviour (players act strategically). More specifically, throughout this monograph we assume that every player believes his opponents to be rational. We distinguish between these two aspects since the first aspect (rationality) applies to every situation in which a person must reach a decision, possibly apart from the context of a game, and the second aspect (acting strategically, believing the opponents to be rational) distinguishes decision theory from game theory: players must act optimally given some conjecture about their opponents' behaviour and be aware of the fact that their opponents will do the same. This conjecture is based on the assumption that 
every opponent is rational too. Consider the following example.

Example 1.1 The students Klaas and Pier have agreed upon having a meal together this enening. Since their preferences concerning food are totally different. they agreed that Klans decides whether they will only have a main dish or a complete menu and Pier decides whether they go to the cheap "Monverland" or to the very exclusive "De Goudfazant". They don't care for the financial aspert; their parents pay for their study and all their meals. Klaas likes very long dinners with very fine food in a nice ambiance and therefore he would prefer the complete menu if they would go to "De Goudfazant". Only having a main dish would just be killing their hunger, so then Klaas doesn't care where they go. However, having a complete menu in Monverland is something he hates: the food is not that good and the ambiance terrible. Klaas' preferences may be represented by the following table.

$$
\begin{array}{cccc}
\multicolumn{3}{c}{\text { Pier }} \\
\text { Klaas main } & 4 & \text { Goudfazant } \\
& \text { menu } & 0 & 4
\end{array}
$$

His friend Pier is a differcnt type of guy: he just likes to eat as much as he can, as long as it doesn't take too much time. He hates the idea of having a menu in "De Goudfazant", sincc he doens't like to sit at a table for hours and getting all different small dishes to eat. Actually he doesn't really care what they eat, as long as he isn't hungry anymore afterwards. The complete menu in Monverland would bo fine to him: it's an "all-you-can-eat-buffet": much and quick. His preferences may be given by

$$
\begin{aligned}
& \text { Pier } \\
& \begin{array}{cccc} 
& & \text { Monverland } & \text { Goudfazant } \\
\text { Klaas main } & 0 & 1 \\
\text { menu } & 1 & 0
\end{array}
\end{aligned}
$$

A frequently used graphical representation of the situation is the following "bimatrix."

$$
\text { Pier }
$$

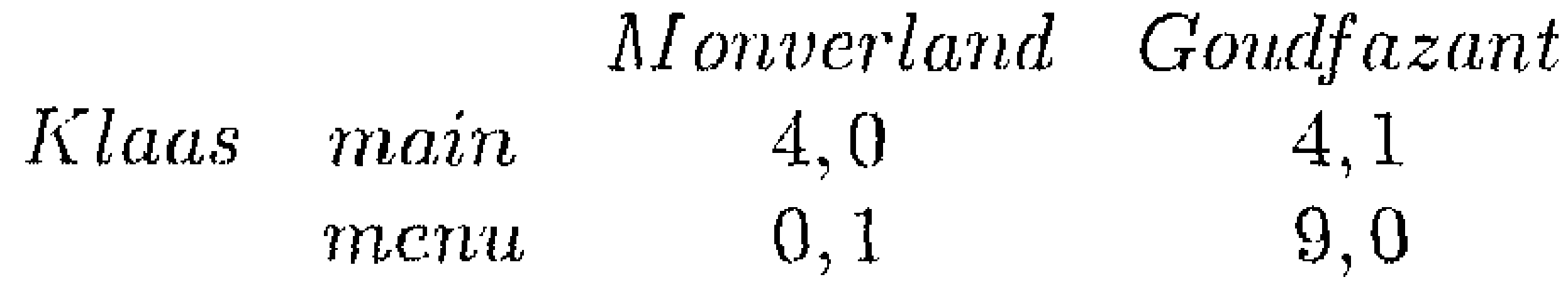

where the first number in an outcome refers to Klaas' utility and the second number to Pier's. Now the question is: what should they decide? And what is a belief? Pier may think that Klaas tends to avoid risks (he is "risk averse") and will prefer to choose only the main dish (always yielding him a utility of 4) over the complete menu (with the possibility of getting utility (0). In this case. Pier will anticipate on his belicf by choosing "De Goudfazant", yielding ulility 1 instead of 0 (by choosing "Monverland"). But of course Pier may also think that Klaas likes the idea of possibly getting utility 9 more than he dislikes the risk of 
getting 0 and chooses the complete menu. Then Pier, holding this belief, would anticipate on his belicf by choosing "Monverland". So Pier's choice depends on what he believes Klaas will choose.

To illustrate how quickly a player in a noncooperative game (or the person who analyses the conflict, such as the reader of this monograph) may get confused by the consequences of the assumptions we just described, consider the following reasoning, based on the same example. Let's consider Pier again. We just assumed that he is rational and acts strategically. But of course the same holds for his "opponent" Klaas. Since Klaas belicves Pier to act strategically he bases his decision on some way of reasoning about his opponent Pier. Hence, the fact that Pier is rational and acts strategically implicitly implies that he must follow some way of reasoning about the way he thinks Klaas thinks that Pier reasons. Of course we can iterate this argument and before we know it we are stuck in "What is optimal for Pier if he thinks that Klaas thinks that Pier thinks that Klaas thinks that Pier thinks that Klaas will behave like this". Perhaps both Klaas and Pier would lose their appetite by these considerations.

Consider again our example. We argued that if Pier holds the belief that his opponent is risk averse (and hence, chooses the main dish) he will choose alternative "De Goudfazant". But if Klaas believes that Pier believes that Klaas chooses the main dish, Klaas belicves that Pier will choose "De Goudfazant". But with that belief, Klaas would be better off by choosing the complete menu, yielding him a utility of 9 instead of 4 .

Pier, on his turn, would prefer "Monverland" over "De Goudfazant" if he believes that Klaas believes that Pier believes that Klaas chooses the main dish. So what must players believe now? And, resulting from these beliefs, which alternative must they choose? Models that consider this iterative kind of reasoning about the opponents' reasoning (resulting in belief hierarchies) are called epistemic models.

We can partly solve (or better, simplify) this problem by assuming that what Klaas believes that Pier believes about how Klaas behaves coincides with how Klaas behaves (and vice versa). So, in terms of our example, if Klaas believes that Pier believes that Klaas will choose the complete menu, Klaas will choose the complete menu. But then, more generally, Klaas must choose a strategy that is optimal against what he believes that Pier chooses. However Pier's choice depends on what Pier believes that Klaas will choose. With the assumption we just described Klaas must choose a strategy that is an optimal response to the strategy that Pier optimally chooses correctly anticipating to Klaas' choice. So players choose strategies that are optimal against each other. These pairs of strategies (a strategy for Klaas and one for Pier that are optimal against each other) are characterized by the property that no player can improve by unilaterally deviating from the strategy pair by choosing a different strategy. This leads to the concept of Nash equilibrium (Nash proved that under certain assumptions such an "equilibrium" always exists). The reader may check (or 
believe) that in our example this is the case if Klaas chooses the main dish and the complete menu both with probability $\frac{1}{2}$ and Pier chooses "Monverland" with probahility $\frac{5}{9}$ and "De Goudfazant" with probability $\frac{4}{9}$. Notice that a strategy may also be a probability distribution. A possible interpretation of such a probability distribution is that it represents a probabilistic belief: an interpretation of Pier's strategy $\left(\frac{5}{9}, \frac{4}{9}\right)$ is that Klaas believes Pier to choose Monverland with probability $\frac{5}{9}$ and Goudfazant with probability $\frac{4}{9}$, rather than that Pier chooses the "strategy" $\frac{5}{9}$ Monverland and $\frac{4}{9}$ Goudfazant (Pier doesn't toss a coin before reaching a (lecision).

Since things get too complicated for them Klaas and Pier decide to give up their idea of having a meal together. Instearl they will both take care of their own meal and have a drink afterwards in either "Monverland" or "De Goudfazant". But now the question arises: shall they drink whisky or beer? They agree that Kilats decides whether they choose whisky or beer and Pier decides where they go. Although they have very generous parents, the drinking part is something they have to pay for themselves. So now a utility function must indicate whether they like the drink more than they dislike the amount of money they have to pay for it. In "Monverland" the beer is "Stella" and the whisky "Glenn Student", whereas in "De Goudfazant" the beer is "Warsteiner" and the whisky the very fine Scotch "Ardberg limited 1990-release". Qualities arc different, Klaas" and Pier's preferences are different and prices are different and we assume that this results in a bimatrix game

\begin{tabular}{cccc} 
& \multicolumn{3}{c}{ Pier } \\
Klaas & Monverland & Goudfazant \\
& beer & 1,5 & 4,4 \\
& whisky & 0,0 & 5,1
\end{tabular}

Pier immediately concludes that (beer, Nonverland) is a Nash equilibrium (no player can gain by milaterally cleviating) and tells Klaas that he has found this Nash ecuilibrium. But Klaas replies that he has found that (whisky, Goudf azant) is a Nash equilibrium too. Of course Pier prefers the first equilibrium and Klaas the second. After doing some calculations they both conclude that the situdtion in which Klaas chooses beer and whisky both with probability $\frac{1}{2}$ and Pier chooses Monverland and Goudfazant both with probability $\frac{1}{2}$ is the third (and last) Nash equilibrium.

Generally, it mighti happen that one player thinks that all players are aiming for one Nash equilibrium, while another player might think that they are aiming for another equilibrium, possibly yielding something they all dislike. Generally, if multiple equilibria exist the concept of Nash equilibrium doesn't give the players a way of reasoning that is guaranteed to lead them towards one of the equilibria: the concept is not self-enforcing in that case.

Since the two "pure" Nash equilibria in the drinking game of Klaas and Pier (in which strategies are played with probability one) are very asymmetric they decide that they can solve the problem by tossing a coin: if the result is 
heads, they will choose (beer, Monverland) and if the result is tail they choose (whisky, Goudfazant). Their expected utilily is

$$
\frac{1}{2}(5,1)+\frac{1}{2}(1,5)=(3,3)
$$

so they both expect to have a utility of 3 . Furthermore, both Pier and Klaas are glad that their agreement is self-enforcing: both options are Nash equilibria, so given the fact that the result of the toss is e.g. heads, every individual player is better of by following the agreement and, hence, has no incentive of deviating from their agreement. So Pier proposes to take this option as a solution of their problem.

Before Klaas can answer their smart colleague Jack interferes. Experience has laught Jack to be as creative as possible, since he has the smallest budget to fulfill his rather large consumption of alcohol. Therefore he proposes the following. He, Jack, will act as a medialor who recommends a strategy to both Klaas and Picr in such a way that each of the pairs

$$
\begin{aligned}
& \text { (beer, Monverland), } \\
& \text { (whisky, Goudfazant), } \\
& \text { (beer, Goudfazant) }
\end{aligned}
$$

is suggested with probability $\frac{1}{3}$. Every player knows the fact that thesc three pairs will be recommended with probability $\frac{1}{3}$ and will only be told the strategy that is recommended to him. The reader may check that following Jack's plan will yield the expected utilities

$$
\frac{1}{3}(5,1)+\frac{1}{3}(1,5)+\frac{1}{3}(4,4)=\left(3 \frac{1}{3}, 3 \frac{1}{3}\right) .
$$

So following Jack's plan would be even better than tossing coins (although Jack demands a part of this difference for his advise and effort to pay for his needs). The only question that remains is whether Jack's advice is self-enforcing in the sense that neither Klaas nor Pier can benefit from cleviating from the recommendation. Assume that Klaas gets the recommendation to choose beer. Then he must believe that Jack recommended both Monverland and Goudfazant to Pier with equal probability $\frac{1}{2}$. Given this consideration, choosing whisky is as good as choosing beer for Klaas (both giving an expected utility of 2,5), so it is optimal to follow Jack's advice. If Jack recommends whisky to Klaas he knows that Jack recommended Goudfazant to Pier and choosing whisky is optimal (yielding utility 5, instead of 4 by deviating). Similar arguments hold for Pier. An equilibrium like this, using a mediator and demanding that it is optimal for every player to follow the mediator's recommendation, is called a correlated equilibrium. Notice that the probability distributution over outcomes with Jack as a mediator can never be achieved as a product of probability distributions that arise from Klaas and Pier choosing strategies with a certain probability independently from each other. 
Let us return to games without communication (using Jack as a mediator was an indirect way of communication between Klaas and Pier about what they were going to choose). Insteal of asking "which strategy should a player choose?" we could also try to focus on the opposite question "which strategy should a player avoid?". To make our point we give an example.

Example 1.2 We look at the following game in which player Alfa chooses between $a, b, c$ and player Omega chooses between $d, e, f$.

\begin{tabular}{ccccc} 
& & \multicolumn{3}{c}{ Omega } \\
& & $d$ & $e$ & $f$ \\
& $a$ & 1,5 & 4,2 & 1,3 \\
Alfa & $b$ & 2,3 & 6,5 & 3,4 \\
& $c$ & 3,2 & 5,3 & 2,5
\end{tabular}

Notice that no matter what player Alfa believes, strategy a is never optimal for him since both $b$ and $c$ yield $A$ a better payoff against any strategy of Omega. So Alfa will never play a (a is "dominated"). For Omega a similar argument at first sight doesn't exclude one of his strategies. However, Omega believes that Alfa is a rational player and hence, Omega believes that Alfa will never play a. But now $d$ is never optimal for Omega since, given the fact that Alfa will never play a, both $e^{2}$ and $f$ yield Omega a belter payoff against any of the remaining strategies of Alfa. If again we would assume some framework of iterative reasoning about the opponents we could take the argument a step further: since Alfa believes that Omega is a rational player and Alfa believes that Omega believes that Alfa is a rational player Alfa belicves that Omega will never choose d. But in the "reduced game", the game that arises if we "eliminate" the strategies a and d, Alfa will always choose prefer $b$ over $c$. The last step in the argument is that now Omega anticipating on this may conclude that $f$ is never optimal for him. So Alfa and Omega reach their conclusion that $(b, d)$ is the only pair that rational players following this iterative process will choose. Notice that $(b, d)$ is also a Nash equilibrium.

If for some fixed strategy $k$ there exists another strategy $l$ such that the ontcome playing $l$ is always strictly better than playing $k$ no matter what the player believes his opponents to decide, the strategy $k$ is called strictly dominated. The process clescribed in the example, where players first eliminate their own strictly dominated strategies and then repeat this procedure in the game arising from the assumption that their opponents will do the same etc. is called iterative elimination of strictly dominated strategies. It can also happen that a strategy is not strictly dominated by any other pure strategy, but is strictly dominated by some mixed strategy (probability distribution), as is illustrated by the following exanple.

Example 1.3 Let the utilities for player 1 in the following game, where 1 
chooses between $a, b, c$ and 2 chooses between $d, e, f$, be given by

$$
\begin{array}{cccc} 
& d & e & f \\
a & 3 & 5 & 9 \\
b & 2 & 8 & 10 \\
c & 10 & 4 & 11
\end{array}
$$

The strategy a is not strictly dominated by any pure strategy, but is strictly dominated by $\frac{1}{2} b+\frac{1}{2} c$.

It may also happen that a game is not given in terms of a utility that is attached to every outcome, but in terms of the preference relation that players hold. By a preference relation we mean some "ranking" that states which outcome of the game is preferred to another outcome of the game. By "outcome" we also include "mixed outcomes", probability distribrutions over pure outcomes. A preference relation is called complete if, no matter which pair of (probability distributions over, as we saw in our example of Nash equilibrium) outcomes, it tells which outcome of the pair is preferred over the other. Otherwise, we say that the preference relation is incomplete. Examples like above of course all describe complete preference relations, since they are given by a utility representation (and thus, we can calculate the expected utility for every strategy choice and belief over the opponenl's strategy choice and compare them). As an example for an incomplete preference relation consider the following situation for some student

$\begin{array}{ccc} & \text { Monverland } & \text { Goudfazant } \\ \text { main } & \text { bad } & \text { ok } \\ \text { menu } & \text { worst } & \text { best }\end{array}$

How must this student compare the following two ontcomes of the game:

1. with probability $\frac{1}{2}$ the outcome is having a menu in "Monverland" and with probability $\frac{1}{2}$ the outcome is having a main clish in "De Goudfazant";

2. having a main dish in "Monverland"

3. the outcome is that the outcomes (menu, goudfazant), (main, goudfazant), (main, monverland) all occur with probability $\frac{1}{3}$ ?

The problem is that the student involved here only knows that one outcome is better compared to another, but doesn't know how much. One way of evaluating situations like this is by first degree stochastic dominance. Here a player cvaluates for every pure outcome the probabilities of getting that outcome or something worse and compares this evaluation for different strategies (he compares cumulative probabilities).

outcome

(menu, monverland)

(main, monverland)

(main, goudfazant)

(menu, goudfazant)

\section{cumulative probability} distribution 1

$\frac{1}{2}$
$\frac{1}{2}$
1
1

distribution 2 distribution 3

$\begin{array}{ll}0 & 0 \\ 1 & \frac{1}{3} \\ 1 & \frac{2}{3} \\ 1 & 1\end{array}$


We say that one distribution dominates another distribution if for every outcome the cunulative probability of the first distribution is smaller than (or equal to) the cumulative probability of the second. The interpretation is that one distribution dominates another if for every outcome the probability of getting this outcome or something worse is smaller for the dominating distribution (hence, the probability of getting something better is larger). In our example, distribution 3 dominates both distribution 1 and 2. Notice that neither distribution 1 dominates distribution 2 nor vice versa. Hence, first degree stochastic dominance induces an incomplete preference relation: distributions 1 and 2 are incomparable by this criterion. A distribution is "undominated" if there exists no other distribution that dominates it. The concept of stochastic dominance can bo defined for higher orders than "first order" and a similar analysis can be performed. Intuitively, if the order of stochastic dominance increases the weight that is put on "bad" outcomes increases, making it harder for distributions putting weight on these bad alternatives to become undominated (compensation by an increasing amount of probability attached to "good" outcomes is necessary). We can also show that as the degree of stochastic dominance increases the preference relation becomes more and more complete (or, more precisely, less and less incomplete).

In our examples until now we assumer that both players choose their strategy simultaneously (they don't anticipate on the opponent's "move"). Of course we can also think of situations in which player 1 may first observe 2's move and then choose his strategy. In such a dynamic game we are directly confronted with the question: what must player 1 think if he observes a decision from 2 that he did not expect? In our "normal language" we have an intuition for what it means that player 2 did not expect some decision of his opponent; let us look at what it means in the somewhat formal context and analyze what we moan by this problem. Player 1 observes a move that he did not expect 2 to play, given 1's belief about 2's preferences and beliefs. Since we "only" imposed two conditions (players are rational and behave strategically in the sense that they believe their opponents to be rational) this may either imply that 2 is not rational (he doesn't play optimally given his beliefs and preferences) or that 2 is playing rationally, but 1 was wrong about 2's preferences and/or beliefs. If we insist on our assumption that all players are rational we are led to the conclusion that 1 must "revise" his bolief about 2's preforences and/or beliefs upon observing the "mexpected move". Hence, 1 must revise his belief such that the observed move becomes rational for 2 .

The outline of this monograph is as follows. In part I we focus on beliefs. First, in chapter 2, we consider "signaling games", in which player two observes a decision that player one takes. Player one, however can be of different types, each possibly holding different preference relations (and thus having different optimal strategies). Player one knows with which probability player one is of which type. We ask ourselves the question: how must player two revise his belief about player one's utility function to rationalize an observed, unexpected 
move. Intuitively it might be clear that this can be clone in many different way, so the next question we ask is: what is the smallest possible revision of player two's belief that rationalizes the observed clecision? Thus, we look for the revised belief that rationalizes the dccision that is as close to the original belief as possible. In orcler to answer this question we must first find some way to measure how close one belief is to another belief. In technical terms we introduce "minimum revision equilibrium" and show that it is a refinement of sequential equilibrium. In chapter 3 we introduce an epistemic model in which players are uncertain about their opponents' utility functions. We assume that every player believos player $i$ 's utility function to be in some class. In epistemic models in which players are certain about the opponents' utility functions (and believe that their opponents are certain about their utility functions etc; the utility functions are commonly known among the players) strategies that survive the process of iterative elimination of strictly dominated strategies play a fundamental role. We show that the same is true for the case in which there is uncertainty about the opponents' utility functions if we impose some further conditions. We introduce closedness under Harsanyi aggregation and show that this condition is sufficient for equivalence between strategies that can rationally be chosen under common belief in rationality (i.e. believing that the opponents are rational, believing that the opponents believe every player to be rational and so on) and strategies that survive the iterative elimination of strictly dominated strategies. As an example we show what this model implies for a generalized Cournot duopoly. In the second part of this dissertation we turn to incomplete preference relations. First, in chapter 4 , we consider "t-th stochastic dominance" as an example for an incomplete preference relation and investigate the possible equilibria for two-person games. Furthermore, we emphasize on the consequences of $t$ (the degree of stochastic dominance) tending to infinity and show that, in some specific cases, this leads to altruism (in the sense that cvery player maximizes the minimal outcome for his opponent). In chapter 5 we consicler incomplete preference relations that satisfy some general notion of aversion towards bad outcomes. First we give an expected utility characterization of these incomplete preference relations by using a result from [Dubra et al, 2004]. Then we look at the game theoretic implications and conclude that in the limit the same behaviour arises as for stochastic dominance. Hence, the results for stochastic dominance are typical for any sequence of preference relations that becomes "infinitely bad outcome averse" in the limit. In chapter 6 we apply the concept of stochastic dominance to correlated equilibria in two-person games and, again, consider the limit for the degree of stochastic dominance tending to infinity (and hence, by chapter 5 , the result also holds for increasingly bad outcome averse preference relations). In order to allow the reader to read some of the chapters independently there will be some overlap in information to make the chapters more self contained. 


\section{Chapter 2}

\section{Revision of conjectures about the opponent's utilities in signaling games}

In this chapter, of which the main results are published in [Schultcis, 2007], we deal with the question what player 2 in a signaling game should believe if he observes a message which he did not expect. Usually, the following assumptions are made:

(i) each player has a utility function that is known to all players throughout the game;

(ii) all players are rational, utility-maximizing players.

In the concept of Nash equilibrium [Nash, 1950] it is required that each player plays optimally given the strategy of his opponent. In the concept of sequential equilibrium [Kreps \& Wilson, 1982] it is additionally required that player 2 has beliefs on information sets that are not reached in equilibrium and decides optimally given these beliefs. However, in signaling games sequential equilibrium does not put any further restrictions on these beliefs. In order to make the concept more powerful, several refinements were introduced in literature, such as perfect sequential equilibrium [Grossman \& Perry, 1086], the intuitive criterion [Cho \& Kreps, 1987] and divine equilibrium [Banks \& Sobel, 1987]. These refinements do put restrictions on player 2's belicfs on information sets that are not reached given the equilibrium. In all these refinements, the idea is that player 2, upon observing an unexpected mossage, makes a distinction between "less plansible" and "more plausible" types, and attaches positive probability only to the more plausible types. Throughout this reasoning process the utility functions are assumed to be fixed, which implies that player 2, upon observing an unexpected message, cannot revise his beliefs about player 1's utility function.

We will follow an alternative path, namely to insist on a player's belief of hav- 


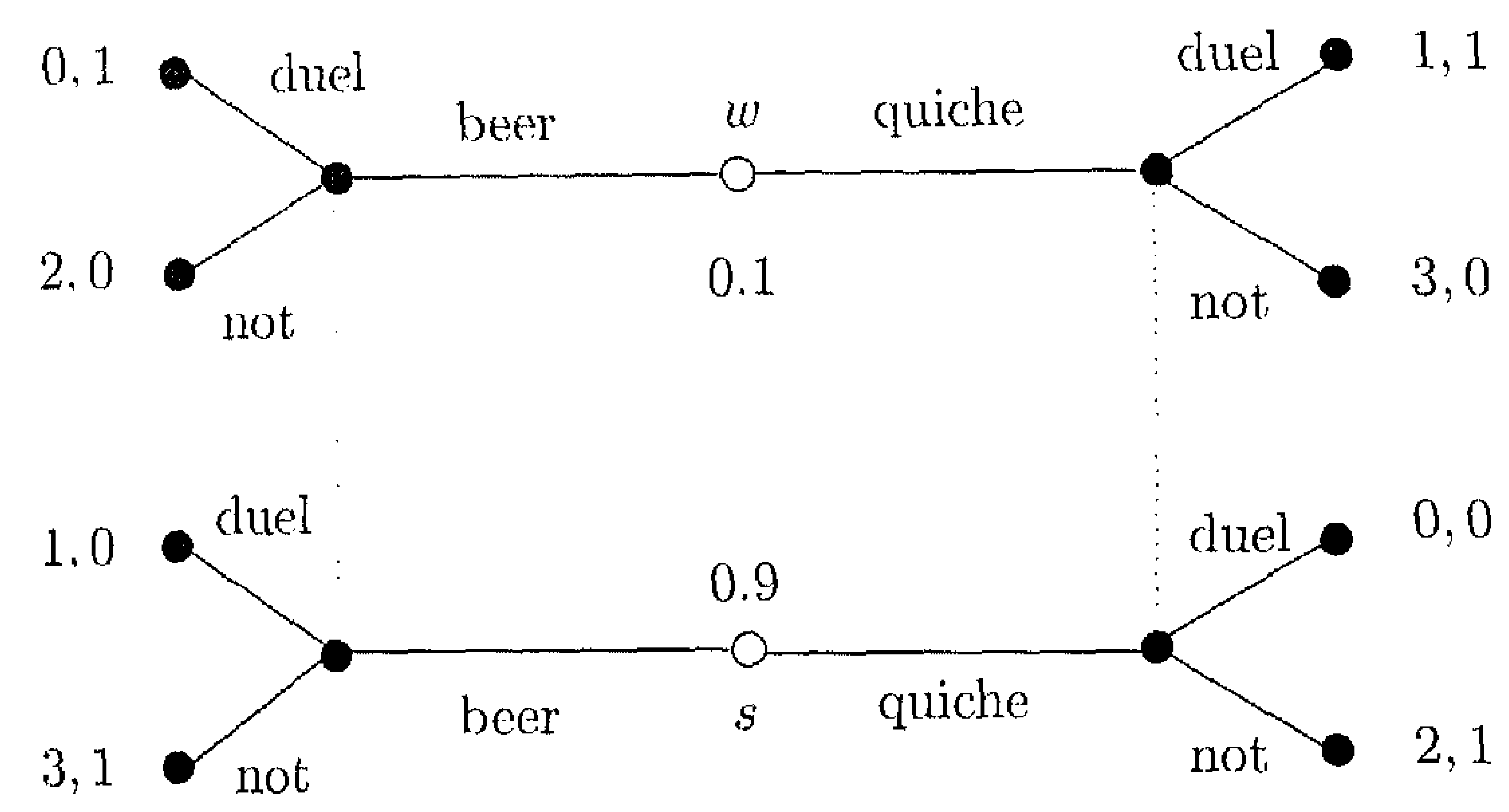

Figure 1

ing a rational opponent and to assume that player 2 has a conjecture about his opponent's utility function, which he may revise after observing player 1's message. This revision should be such that the given message becomes optimal for player 1 . This leads to the concept of preference conjecture cquilibrium, first formalized in [Perea, 2003].

It is important at this stage to compare preference conjecture equilibrium to rationality concepts that have been defined for situations where beliefs about the opponent's utility functions cannot be revised. As a benchmark for the latter, consider the concept of sequential equilibrium. In the concept of sequential equilibrium applied to signaling games, player 2 has beliefs about player 1's types. In contrast, in the concept of preference conjecture equilibrium player 2 holds, at the beginning of the game and after observing player 1's message, a belief about his opponent's utility function as well as a belief about the opponent's strategy choice. Furthermore, we demand that player 1's expectation about player 2's strategy choice coincides with player 2's strategy choicc. When player 2 observes a message sent by his opponent that would have been suboptimal for player 1 given player 2's expectations at the beginning of the game, player 2 revises his belief about player 1's utility function. These changes are inevitable once we insist on the assumption that the players are rational and expect their opponent to be rational too. The profile of beliefs that emerges from this procedure, for every player on every information set, is called a conjecture profile.

We show that for signaling games the concepts of preference conjecture equilibrium and sequential equilibrium are in some sense equivalent. As an illustration, consider the game in Figure 1. This is the well known beer-quiche game introduced by Cho and Kreps. At the beginning of the game nature decicles between weak $(\mathrm{w}$ ) and strong (s) as types for player 1 with probabilities 0.1 and 0.9 respectively. Then player 1 chooses between the messages beer and quiche. 
Player 2 observes this chosen message but not player 1's type. Based on his observation he subsequently decides to either challenge player 1 for a duel or not. This game possesses two kinds of sequential equilibria, namely one in which every type of player 1 chooses beer and one in which player 1 always chooses quiche. The intuitive criterion (sec [Cho \& Kreps, 1987]) exclucles equilibria of the second kind by the following argument. If player 2 expects both types to choose quiche, then he chooses not to duel after quiche. But what if player 2 observes beer? He concludes that it is the strong type that deviated, since the weak type can get at most 2 by deviating, which is less than 3 , which he gets by choosing quiche and the strong type could get 3 by deviating, which is more than the 2 he gets by choosing quiche. Hence, player 2 will choose to avoid the duel after observing beer. Then however, the strong type will deviate from the equilibrium by choosing beer. Hence, the intuitive critcrion excludes the second type of equilibrium, because it is supported by the belief that player 1 is weak if he drinks beer, in spite of the fact that the weak type always obtains less than its equilibrium payoff 3 by deviating. To illustrate the concept of preference conjecture equilibrium, we now consider the depicted utilities as the players' conjectures about their opponent's utility function at the beginning of the game. Furthermore we let the conjectures about the opponent's strategy choice be such that player 2 initially believes that both types of player 1 will choose quiche and playcr 1 expects that player 2 will duel after observing beer and not duel after observing quiche. So the question is: what should player 2 decide if he observes beer? By deviating from player 2's expectation, the weak type now has a utility of 0 , compared to 3 if he would have chosen quiche. In order to rationalize this, we might assume that player 2 thinks the weak type's payoff will be 4 after deviating. Thus player 2 will think the weak type's preference relation is such that the weak type prefers beer over quiche, in contrast to what he believed at the beginning of the game. Of course, player 2 may also believe that only the strong type has chosen beer after observing the message beer and may thus choose not to duel. But in that case the strong type would improve his utility by deviating from the initial conjecture and we will show that these conjectures cannot be extended to a preference conjecture equilibrium. To illustrate the relationship with sequential equilibrium, we see that both types taking quiche can only lead to an equilibrium if player 2 , after observing beer, believes it was the weak type that deviated with a probability of at least 0.5. Thus playcr 2 might also believe that both types had some incentive to deviate, since he also underestimated the strong type's payoff after duelling and getting his favorite breakfast. Because of the same argument, he will have to choose to duel, so his conjecture about player 1's utility function after beer and duel might be 4 for the woak type and 5 for the strong type. It is, however, very intuitive to impose the condition that the revisions should be as limited as possible, which means that the revised conjectures should bo as close as possiblo to the initial conjecture. The question arises how to measure the revisions and what the requirement of minimality of these revisions implies.

In this chapter we introduce the concept of minimum revision equilibrium, a re- 
finement of preference conjecture equilibrium that imposes further restrictions on the belief revisions based on the revision index. Consider again the game in Figure 1. In games with only two messages we consider the number of types for which player 2 changes his belief about their preference relation over messages. When we discussed some preference conjecture equilibria of this game, we saw that in the first onc, where we assumed that after observing beer, player 2 believes that only the weak type has chosen beer. In this case player 2 only lias to change his belief about the weak type's preference relation over becr and quiche. In the second preference conjecture equilibrium, where we assumed that after observing beer, player 2 believes that both types liave chosen beer, player 2 has to cliange his belief over both types' preference relations over beer and quiche. We thus say that the revision indices are 1 and 2 respectively. In order to determine the minimum revision equilibrium, we compute this inclex for all preference conjecture equilibria and choose a preference conjecture equilibrium with the minimal index. Since preference conjecture equilibrium will be shown to coincide with scquential equilibrium for signaling games this implies that minimum revision equilibrium can be interpreted as a refinement of sequential equilibrium. We find in the example above that there are two minimum revision equilibria, both with a revision index of one. The first one corresponds to the sequential equilibrium in which both types choose quiche and player 2 , when observing beer, attaches probability one to the fact that the weak type doviated. The second minimum revision equilibrium corresponds to the sequential equilibrium in which both types choose beer and, after observing quiche, player 2 attaches probability one to the fact that the weak type deviated. We see that one of these minimum revision equilibria coincides with the equilibrium that is selected by the intuitive criterion, but the other one does not.

The chapter is organized as follows. In section 1 we develop the concept of preference conjecture equilibrium for signaling games. In section 2 we investigate the relationship with sequential equilibrium, established by Theorems 2.10 and 2.12. We show that every preference conjecture cquilibrium induces a sequential equilibrium and that vice versa every sequential equilibrium corresponds to a preference conjecture equilibrium. In section 3 we introduce the minimum revision equilibrium and elaborate on its application as a refinement of sequential equilibrium in signaling games. In section 4 we provide some examples and compare the concept of minimum revision equilibrium to the intuitive criterion. The last section, section 5 , elaborates on the structure of the set of sequential equilibria in signaling games. We show that this set can be written as a union of sets on which the revision index is constant. These sets can be computed by solving systems of linear inequalities, which facilitates the computation of minimum revision equilibria.

\subsection{Preliminaries}

For a finite set $Q, \Delta(Q)$ denotes the set of all probability distributions over $Q$, and $\Delta^{0}(Q)$ denotes the set of completely mixed probability clistributions over 
$Q$.

Definition 2.1 A signaling game is a tuple $\mathcal{S}=\left(T, M . A, p, u_{1}, u_{2}\right)$ where $T$. $M$ and $A$ are finite sets, $p$ is an element of $\Delta^{\prime}(T)$, and $u_{1}$ and $u_{2}$ are functions from $T \times M \times A$ to $\mathbb{R}$.

$\Lambda$ signaling game is played as follows:

- Nature selects a type $t$ of player 1 from the set $T$ of types. Type $t$ is selected with probability $p(t)>0$.

- Player 1 , knowing his type, chooses a message $m$ from the set of messages $M I$.

- Player 2 , not knowing the type of player 1 , but knowing the probability distribution $p$, observes the message $m$ and subsequently chooses an action a from the set $A$.

- The utilities are $u_{1}(t, m, a)$ for player 1 and $u_{2}(t, m, a)$ for player 2 .

In the usual extensive form representation of a signaling game, each decision node of player 1 corresponds to a type $t$. Each clecision node of player 2 corresponds to a pair $(t, m)$. A terminal node corresponds to a triple $(t, m, a)$. An information set of player 2 corresponds to a message $m$. Henceforth, we use the notation fixed in Definition 2.1 for a signalling game, unlcss stated otherwise. The following example is used for illustrative purposes throughout this chapter.

Example 2.2 consider the signaling game in Figure 2. Player 1 can be of two types $t_{1}$ and $t_{2}$, each with probability $\frac{1}{2}$. Player 1 chooses between three messages $k, l$ and $m$, and player 2 chooses between the actions $a$ and $b$. The numbers at the terminal nodes are the utilities of player 1 and 2 , respectively.

\subsubsection{Sequential equilibrium}

A pure strategy for player 1 in a signaling game is a map $s_{1}: T \rightarrow M$, defining a messiage $m$ for every type $t$. The sct of pure strategies for player 1 is denoted by $S_{1}$. An element $\sigma_{1} \in \Delta\left(S_{1}\right)$ is called a mixed strategy for player 1 . So, $\sigma_{1}\left(s_{1}\right)$ is the probability of the pure stratcgy $s_{1}$. Similarly, a pure strategy for player 2 is a map $s_{2}: M I \rightarrow A$, defining an action a for every message $m$. The set of pure strategies for player 2 is clenoted by $S_{2}$. An element $\sigma_{2} \in \Delta\left(S_{2}\right)$ is called a mixed strategy for player 2 . So, $\sigma_{2}\left(s_{2}\right)$ is the probability of the pure strategy $s_{2}{ }^{1}$.

A system of beliefs (for player 2) is a collection $\beta=(\beta(m))_{m \in M}$ where $\beta(m)=$ $(\beta(t \mid m))_{t \in T} \in \Delta(T)$ for each $m \in M$. As usual, $\beta(t \mid m)$ is the probability that

\footnotetext{
${ }^{1}$ For our purposes, it is convenient to work with mixed strategies instead of bchavioral strategies. In Section 6 we also use behavioral strategies.
} 

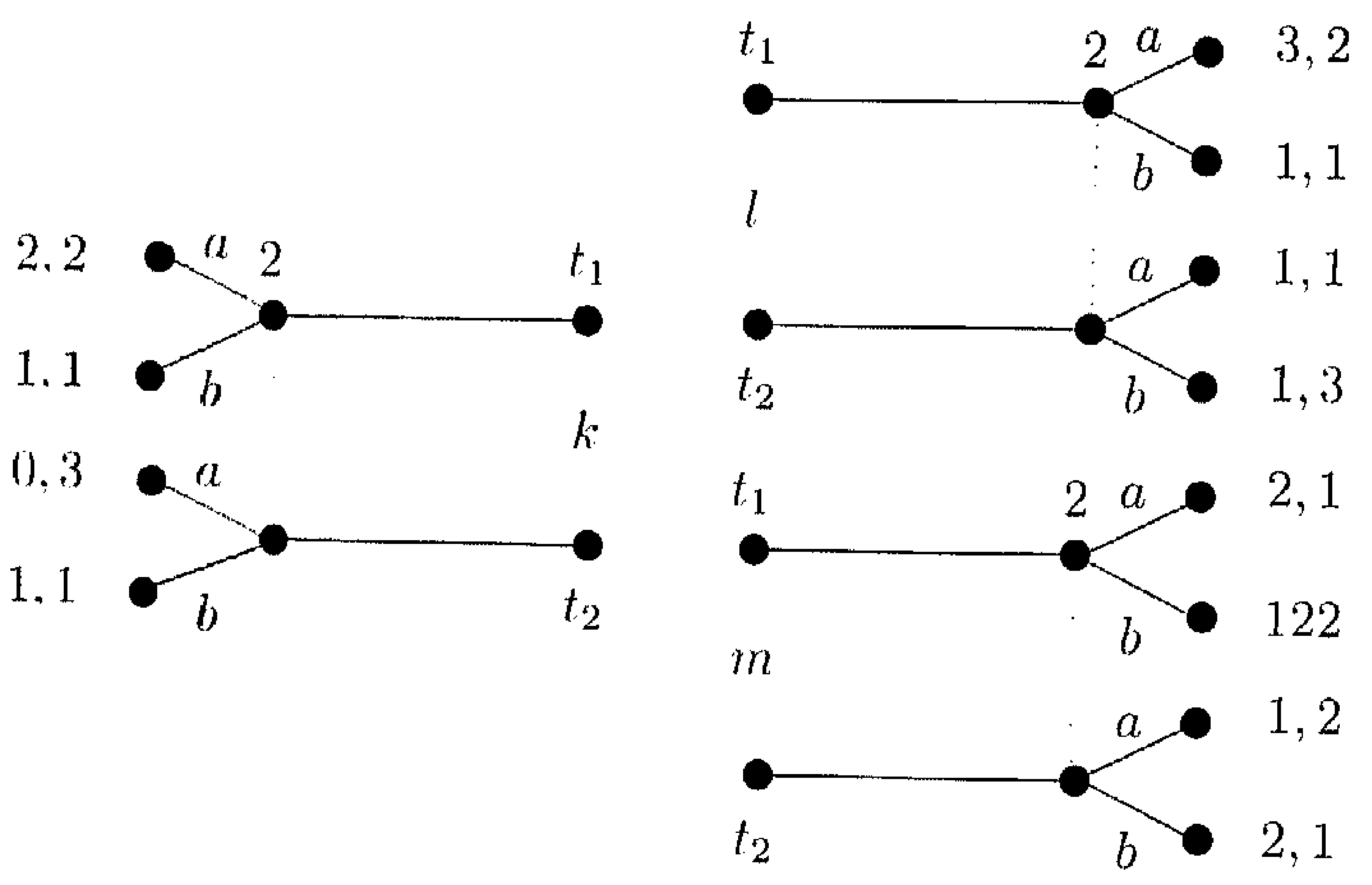

Figure 2: Example 2.2 
player 2 attaches to player 1 being of type $t$ if message $m$ is played. For a mixed strategy $\sigma_{1} \in \Delta\left(S_{1}\right)$ lot

$$
\sigma_{1}(m \mid t):=\sum_{s_{1} \in S_{1}: s_{1}(t)=m} \sigma_{1}\left(s_{1}\right)
$$

be the probability that player 1 plays $m$ if he is of type $t$. The pair $\left(\sigma_{1}, \beta\right)$ is called Bayesian consistent if for all types $t$

$$
\beta(t \mid m)=\frac{p(t) \cdot \sigma_{1}(m \mid t)}{\sum_{t^{\prime} \in T} p\left(t^{\prime}\right) \cdot \sigma_{1}\left(m \mid t^{\prime}\right)}
$$

for all messages $m$ such that $\sum_{l^{\prime} \in T} p\left(t^{\prime}\right) \cdot \sigma_{1}\left(m \mid t^{\prime}\right)$ is positive. Hence, Bajresian consistency puts no restrictions on beliefs on information sets that are reached with probability zero if $\sigma_{1}$ is played. In signaling games, Bayesian consistency is equivalent to consistency as defined in [Kreps \& Wilson, 1982]. A proof of this statement can be found in section 2.6 .

A triple $\left(\sigma_{1}, \sigma_{2}, \beta\right) \in \Delta\left(S_{1}\right) \times \Delta\left(S_{2}\right) \times \Delta(T)^{M I}$ is called an asscssment. For such an assessment

$$
U_{1}\left(t, s_{1}, \sigma_{2}\right):=\sum_{s_{2} \in S_{2}} \sigma_{2}\left(s_{2}\right) \cdot u_{1}\left(t, s_{1}(t), s_{2}\left(s_{1}(t)\right)\right)
$$

is the expected utility for player 1 if his type is $t$, he plays $s_{1}$ and player 2 plays $\sigma_{2}$, and

$$
U_{2}\left(m, s_{2}, \beta\right):=\sum_{t \in T} \beta(t \mid m) \cdot u_{2}\left(t, m, s_{2}(m)\right)
$$

is the expected utility for player 2 if he observes message $m$, has belief $\beta$ and plays $s_{2}$. We call $s_{1} \in S_{1}$ optimal with respect to $\sigma_{2}$ if

$$
U_{1}\left(t, s_{1}, \sigma_{2}\right) \geq U_{1}\left(t, s_{1}^{\prime}, \sigma_{2}\right)
$$

for all $s_{1}^{\prime} \in S_{1}$ and all types $t$. For $m \in M$, we call $s_{2} \in S_{2}$ optimal with respect to $\beta$ on $m$ if

$$
U_{2}\left(m, s_{2}, \beta\right) \geq U_{2}\left(m, s_{2}^{\prime}, \beta\right)
$$

for all $s_{2}^{\prime} \in S_{2}$. We call $s_{2} \in S_{2}$ optimal with respect to $\beta$ if $s_{2}$ is optimal with respect to $\beta$ on every $m \in M$.

We say that $\sigma_{1}$ is optimal with respect to $\sigma_{2}$ if, for all $s_{1} \in S_{1}, \sigma_{1}\left(s_{1}\right)>0$ implies that $s_{1}$ is optimal with respect to $\sigma_{2}$. We call $\sigma_{2}$ optimal with respect to $\sigma_{1}$ if, for all $s_{2} \in S_{2}$ and all $m \in M$ with $\sum_{t^{\prime} \in T} p\left(t^{\prime}\right) \sigma_{1}\left(m \mid t^{\prime}\right)>0, \sigma_{2}\left(s_{2}\right)>0$ implies that $s_{2}$ is optimal with respect to $\beta$ on $m$, where $\beta$ satisfies (2.1). We call $\sigma_{2}$ optimal with respect to $\beta$ if, for all $s_{2} \in S_{2}, \sigma_{2}\left(s_{2}\right)>0$ implies that $s_{2}$ is optimal with respect to $\beta$.

Definition 2.3 The assessment $\left(\sigma_{1}, \sigma_{2}, \beta\right)$ is a (Bayesian) Nash equilibrium if 
(i) $\left(\sigma_{1}, \beta\right)$ is Bays sian consistent;

(ii) $\sigma_{1}$ is optimal with respect to $\sigma_{2}$;

(iii) $\sigma_{2}$ is optimal with respect to $\sigma_{1}$.

If, monesoner, $\sigma_{2}$ is optimal to $\beta$. then $\left(\sigma_{1}, \sigma_{2}, \beta\right)$ is a sequential equilibrium ${ }^{2}$.

The? difference betwen (Bayesian) Nash (cf. Nash, 1950, 1951; Harsanyi, 1967, 1968) and soruential (c.f. Kreps and Wilson, 1982) equilibrium is that in the latter player 2 also plays optimally on information sets reached with zero probability.

\subsubsection{Preference conjecture equilibrium}

The concent of prefornec conjecture equilibriumwas introduced in [Perea, 2003] for general games in extensive form. In this chapter we study this concept for signaling ganuss".

In a conjecture profile each player has a conjecture about the opponent's stratfog choice and a conjecture about the opponent's utility function. For player 1. these conjectures are made at the beginning of the game: since player 1 moves first and only once, it makes no sense to consider revisions of player 1 's conjectures. For player 2 the situation is different. Player 2 holds conjectures at the beginning of the ganne but may revise these after observing player 1 's move. A conjecture profile entails induced conjectured preferences of the players. In a preforence conjecture equilibrium the players believe each other to play rationaly - that is to maximize their conjectured preferences-implying that player 2 may have to revise his conjectures about player 1 . Furthermore, revisions of conjectures about player 1 's strategies will be restricted by imposing Bayesian updating.

Formally: starting with player 1 , let $\mu_{12} \in \Delta\left(S_{2}\right)$ be his conjecture (at the begiming of the game) about player 2 s strategy choice. Thus, $\mu_{12}\left(s_{2}\right)$ is the probability player 1 assigns to player 2 playing strategy $s_{2}$. Similarly, let $u_{12}$ : $T \times M \times A \rightarrow \mathbb{R}$ be player 1 's conjecture about player 2 's utility function. Denote $c_{12}=\left(\mu_{12} \cdot u_{12}\right)$.

As explained above, for player 2 the situation is different. He holds conjectures both at the begiming of the game and at every information set $m \in M$ : drpending on the ofserved message, player 2 may revise the conjecture held at the beginning. To denote conjectures of player 2 at the beginning of the game: it is converient to introduce the symbol $h_{0}$. Thus, for the beginning of the game we denote $S_{1}\left(h_{0}\right):=S_{1}$ and for $m \in M$ we denote $S_{1}(m):=$ $\left\{s_{1} \in S_{1} \mid\right.$ there exists $t \in T$ with $\left.s_{1}(t)=m\right\}$. Let $M^{*}:=M \cup\left\{h_{0}\right\}$. For all $m \in N^{*}$. let $\mu_{21}(m) \in \Delta\left(S_{1}(m)\right)$ be player 2 's conjecture at $m$ about player 1 's

\footnotetext{
2In the literature this form of optimality is also called sequential rationality, see Kreps and Wiknn (1982).

"In Pera (20):3) the variant of preforence conjecture equilibrium that we discuss here is

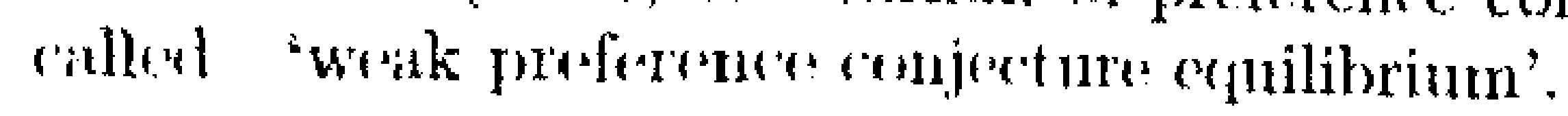


strategy choice. For $s_{1} \in S_{1}(m), \mu_{21}(m)\left(s_{1}\right)$ is the probability player 2 assigns at $m$ to player 1 playing $s_{1}$. Similarly, for all $m \in M^{*}$ let $u_{21}(m): T \times M I \times A \rightarrow \mathbb{R}$ be player 2's conjecture at $m$ about player 1 's utility function. For all $m \in I^{*}$ denote $r_{21}(m)=\left(\mu_{21}(m), u_{21}(m)\right)$.

Definition 2.4 A combination $c:=\left(c_{12},\left(c_{21}(m)\right)_{m \in M^{*}}\right)$ is called a conjecture profile.

In a preference conjecture equilibrium a conjecture profile plays a role similar to that of an assessment in a sequential equilibrium. Also for a conjecture profile we introduce optimality conditions. We start, however, with a condition implying that player 2 revises his conjecture about player 1's strategy according to Bayes' rule, whenever possible.

Definition 2.5 A conjecture profile satisfies Bayesian updating if

$$
\mu_{21}(m)\left(s_{1}\right)=\frac{\mu_{21}\left(h_{0}\right)\left(s_{1}\right)}{\sum_{s_{1}^{\prime} \in S_{1}(m)} \mu_{21}\left(h_{0}\right)\left(s_{1}^{\prime}\right)}
$$

for all $s_{1} \in S_{1}(m)$ and all $m \in M$ such that $\sum_{s_{1}^{\prime} \in S_{1}(m)} \mu_{21}\left(h_{0}\right)\left(s_{1}^{\prime}\right)>0$.

The next task is to define optimality on the parts of both players in a conjecture profile. For this we use that, given their conjectures, players can compute their opponent's expected utility.

Let $m \in M^{*}$. Player 2 can compute his conjecture at $m$ about player 1 's expected utility if player 1 has type $t$, conjecture $\mu \in \Delta\left(S_{2}\right)$ about player 2's strategy choice, utility function $u_{21}(m)$, and plays $s_{1}$, as

$$
U_{21}\left(t, s_{1}, u_{21}(m), \mu\right):=\sum_{s_{2} \in S_{2}} \mu\left(s_{2}\right) \cdot u_{21}(m)\left(t, s_{1}(t), s_{2}\left(s_{1}(t)\right)\right) .
$$

In order to justify the next step in our argument we make the assumption that conjectures are common belief among the players. This means that a player's (informal) conjecture about an opponent's conjecture coincides with this opponent's conjecture. In particular, this implies that player 2's conjecture about player 1's conjecture about player 2's strategy choice coincides with player 1's conjecture about player 2's strategy choice. Therefore it makes sense to evaluate the above conjecture of player 2 at $m$ about player 1 's expected utility at $\mu=\mu_{12}$. Thus we write

$$
U_{21}\left(t, s_{1}, u_{21}(m), \mu_{12}\right)=\sum_{s_{2} \in S_{2}} \mu_{12}\left(s_{2}\right) \cdot u_{21}(m)\left(t, s_{1}(t), s_{2}\left(s_{1}(t)\right)\right) .
$$

A strategy $s_{1}$ is optimal for player 1 with respect to $\mu_{12}$ and $u_{21}(m)$ if

$$
U_{21}\left(t, s_{1}, u_{21}(m), \mu_{12}\right) \geq U_{21}\left(t, s_{1}^{\prime}, u_{21}(m), \mu_{12}\right)
$$

for all $s_{1}^{\prime} \in S_{1}$ and all $t \in T$. 
Sinilarly, consider a pure strategy $s_{2}$ of player 2 , a utility function $u_{12}$ as well as a probability distribution $\mu^{\prime} \in \Delta\left(S_{1}(m)\right)$, where now $m \in M$ (so $m \neq h_{0}$ : see Remark 2.7 below). Then player 1 holds a conjecture about player 2 's expected utility at $m$ given by

$$
U_{12}\left(m, s_{2}, u_{12}, \mu^{\prime}\right):=\sum_{t \in T} p(t) \sum_{s_{1}: s_{1}(t)=m} \mu^{\prime}\left(s_{1}\right) \cdot u_{12}\left(t, m, s_{2}(m)\right) .
$$

Again because of the assumption of common belief of conjectures it makes sense to evaluate this at $\mu^{\prime}=\mu_{21}(m)$ :

$$
U_{12}\left(m, s_{2}, u_{12}, \mu_{21}(m)\right)=\sum_{t \in T} p(t) \sum_{s_{1}: s_{1}(t)=m} \mu_{21}(m)\left(s_{1}\right) \cdot u_{12}\left(t, m, s_{2}(m)\right) .
$$

The strategy $s_{2}$ is optimal for player 2 with respect to $\mu_{21}(m)$ and $u_{12}$ if

$$
U_{12}\left(m, s_{2}, u_{12}, \mu_{21}(m)\right) \geq U_{12}\left(m, s_{2}^{\prime}, u_{12}, \mu_{21}(m)\right)
$$

for all $s_{2}^{\prime} \in S_{2}$.

Definition 2.6 A conjecture profile c satisfies optimality if:

1. for all $m \in M^{*}$ and for all $s_{1} \in S_{1}$, if $\mu_{21}(m)\left(s_{1}\right)>0$, then $s_{1}$ is optimal for player 1 with respect to $\mu_{12}$ and $u_{21}(m)$;

2. for all $m \in M$ and for all $s_{2} \in S_{2}$, if $\mu_{12}\left(s_{2}\right)>0$, then $s_{2}$ is optimal for player 2 with respect to $\mu_{21}(m)$ and $u_{12}$.

Remark 2.7 Observe that, in this definition, optimality on the part of player 2 is not required on $h_{0}$, that is, at the beginning of the game: it is not hard to verify that this is taken care of by the second part of the definition combined with Bayesian updating.

Now, we are sufficiently equipped to define the main concept of this chapter.

Definition 2.8 A conjecture profile $c$ is a preference conjecture equilibrium if it satisfies optimality and Bayesian updating.

Example 2.9 As an illustration, consider Example 2.2. Consider the conjecture profile $c$, where $\mu_{12}$ attaches probability 1 to strategy $(a, a, a)$ and $u_{12}$ coincides with player 2's utilities in Figure 2. Let $\mu_{21}\left(h_{0}\right), \mu_{21}(l)$ and $\mu_{21}(m)$ attach probability 1 to strategy $(l, m)$, and let $\mu_{21}(k)$ attach probability 1 to strategy $(k, m)$. Let $u_{21}\left(h_{0}\right), u_{21}(l)$ and $u_{21}(m)$ coincide with player 1 's utilities in Figure 2, and let $u_{21}(k)\left(t_{1}, k, a\right)=5$ and $u_{21}(k)\left(t^{\prime}, m^{\prime}, a^{\prime}\right)=u_{21}\left(h_{0}\right)\left(t^{\prime}, m^{\prime}, a^{\prime}\right)$ for all $\left(t^{\prime}, m^{\prime}, a^{\prime}\right) \neq\left(t_{1}, m, a\right)$. This conjecture profile is actually a preference conjecture equilibrium and can be interpreted as follows. Given his conjecture $\mu_{21}\left(h_{0}\right)$, player 2 initially expects message $k$ not to be chosen. However, if he obsermes message $k$, he revises his conjecture about player 1 's strategy choice and utility function in such a way that the choice $k$ becomes optimal for at least 
one of the types of player 1 . In this conjecture profile c, player 2 does so by raising the ulility for type $t_{1}$ after message $k$ and action a, while keeping all other utilities unchanged. Thus player 2 , after observing message $k$, believes that it was lype $t_{1}$ and not t. who sent this message. Hence player 2, after observing $k$, assigns probability 1 to the strategy in which type $t_{1}$ chooses message $k$ and type $t_{2}$ still sends message $m$.

\subsection{Relation with sequential equilibrium}

In this section we investigate the relation between preference conjecture equilibria and sequential cquilibria in a signaling game. We show that in a signaling game each preference conjecture equilibrium incluces in a natural way a sequential equilibrium and that converscly each sequential equilibrium is induced this way by at least one preference conjecture equilibrium. In particular this shows that a preferencc conjecture equilibrium always exists (by cxistence of sequential equilibrium).

Consider a conjecture profile

$$
c=\left(c_{12},\left(c_{21}(m)\right)_{m \in N^{*}}\right)=\left(\mu_{12}, u_{12},\left(\mu_{21}(m), u_{21}(m)\right)_{m \in I^{*}}\right) .
$$

Is there an intuitive way to associate with $c$ an assessment $\left(\sigma_{1}, \sigma_{2}, \beta\right)$ ? Note that, for example, $\mu_{21}\left(h_{0}\right)$ is player 2's conjecture at the beginning of the game about player 1's strategy choice. Since we assumed common belief of conjectures among players, we know that player 1's (informal) conjecture about $\mu_{21}\left(h_{0}\right)$ coincides with $\mu_{21}\left(h_{0}\right)$. Hence, for equilibrium analysis it seems intuitivc to put $\sigma_{1}$ equal to $\mu_{21}\left(h_{0}\right)$. In other words, if we interpret $\sigma_{1}$ as the strategy that player 1 actually plays, then this is equal to what player 1 thinks that player 2 thinks that player 1 plays. By a similar line of reasoning it seems intuitive to put $\sigma_{2}$ equal to $\mu_{12}$. Thus,

$$
\sigma_{1}=\mu_{21}\left(h_{0}\right) \text { and } \sigma_{2}=\mu_{12}
$$

Also, for the beliefs $\beta$ held by player 2 , it is natural to let

$$
\beta(t \mid m)=\frac{\sum_{s_{1}: s_{1}(t)=m} p(t) \cdot \mu_{21}(m)\left(s_{1}\right)}{\sum_{t^{\prime} \in T} \sum_{s_{1}: s_{1}\left(t^{\prime}\right)=m} p\left(t^{\prime}\right) \cdot \mu_{21}(m)\left(s_{1}\right)} .
$$

for all $t \in T$ and $m \in M$ (note that the denominator is always positive). Hence, The definitions in (2.2) state that in the induced sequential equilibrium a player should actually play what his opponent conjectures him to play in the preference conjecture equilibrium. Given these choices, the equality (2.3) simply follows from Bayesian updating.

We now say that the assessment $\left(\sigma_{1}, \sigma_{2}, \beta\right)$, clefined by (2.2) and (2.3), is induced by the conjecture profile $c$. One may note that, informally speaking, the assumption of common belief in the conjecture profile $c$ justifies the usual assumption of common knowledge for the induced assessment $\left(\sigma_{1}, \sigma_{2}, \beta\right)$. 
Theorem 2.10 Let $\mathcal{S}=\left(T . M . A . p, u_{1}, u_{2}\right)$ be a signaling game and let $c$ be a preference conjecture equilibrium with $u_{21}\left(h_{0}\right)=u_{1}$ and $u_{12}=u_{2}$. Then the assessment indued by c is a sequential equilibrium in $\mathcal{S}$.

Proof. We first shuw that $\sigma_{1}$ is optimal with respect to $\sigma_{2}$. Take a type $t \in T$. a pure strategy $s_{1} \in S_{1}$ with $\sigma_{1}\left(s_{1}\right)>0$ and an arbitrary strategy $r_{1}^{\prime} \in S_{1}$. It sulfices to show that

$$
U_{1}\left(t, s_{1}, \sigma_{2}\right) \geq U_{1}\left(t, s_{1}^{\prime}, \sigma_{2}\right) .
$$

To this cond. notice that also $\mu_{21}\left(h_{0}\right)\left(s_{1}\right)>0$, because $\sigma_{1}=\mu_{21}\left(h_{0}\right)$. So, because $r$ is a preference conjecture equilibrium, it follows that $s_{1}$ is optimal with respect to $\mu_{12}$ and $u_{21}\left(h_{0}\right)$. In particular,

$$
U_{21}\left(h_{0}\right)\left(t, s_{1}, \mu_{12}\right) \geq U_{21}\left(h_{0}\right)\left(t, s_{1}^{\prime}, \mu_{12}\right) .
$$

With $u_{21}\left(h_{11}\right)=u_{1}$ and $\sigma_{2}=\mu_{12}$ this means exactly that $U_{1}\left(t, s_{1}, \sigma_{2}\right) \geq$ $U_{1}\left(t, x_{1}^{\prime}, \sigma_{2}\right)$.

Next. we show that $\sigma_{2}$ is optimal with respect to $\beta$. Let $m$ be a message, take at pure strategy $s_{2}$ with $\sigma_{2}\left(s_{2}\right)>0$ and a pure strategy $s_{2}^{\prime}$. Again it suffices to show that

$$
U_{2}\left(m, s_{2}, \beta\right) \geq U_{2}\left(m, s_{2}^{\prime}, \beta\right)
$$

Since $\mu_{12}\left(s_{2}\right)=\sigma_{2}\left(s_{2}\right)>0$ and $c$ is a preference conjecture equilibrium, we know that $s_{2}$ is optimal w.r.t. $\mu_{21}(m)$ and $u_{12}$. So, in particular

$$
U_{12}\left(m, \mu_{21}(m), s_{2}\right) \geq U_{12}\left(m, \mu_{21}(m), s_{2}^{\prime}\right) .
$$

However. using the definition of $\beta(t \mid m)$, rearranging torms shows that

$$
\begin{aligned}
& U_{12}\left(m . \mu_{21}(m), s_{2}\right)=\left[\sum_{t^{\prime} \in T} \sum_{s_{1}: s_{1}\left(t^{\prime}\right)=m} p\left(t^{\prime}\right) \cdot \mu_{21}(m)\left(s_{1}\right)\right] \cdot U_{2}\left(m, s_{2}, \beta\right) \\
& U_{12}\left(m, \mu_{21}(m), s_{2}^{\prime}\right)=\left[\sum_{t^{\prime} \in T} \sum_{s_{1}: s_{1}\left(t^{\prime}\right)=m} p\left(t^{\prime}\right) \cdot \mu_{21}(m)\left(s_{1}\right)\right] \cdot U_{2}\left(m, s_{2}^{\prime}, \beta\right)
\end{aligned}
$$

Hence. since the bracketed factor is always positive and the same for both $s_{2}$ and $s_{2}^{\prime}$, we see that $U_{2}\left(m, s_{2}, \beta\right) \geq U_{2}\left(m, s_{2}^{\prime}, \beta\right)$.

Finally; we show that $\beta$ is Bayesian consistent with $\sigma_{1}$. Take a message $m$ and a type $t$, and assume that $\sum_{t^{\prime} \in T} p\left(t^{\prime}\right) \sigma_{1}\left(m \mid t^{\prime}\right)>0$. We will show that

$$
\beta(t \mid m)=\frac{p(t) \cdot \sigma_{1}(m \mid t)}{\sum_{t^{\prime} \in T} p\left(t^{\prime}\right) \cdot \sigma_{1}\left(m \mid t^{\prime}\right)} .
$$

Fom the assumption it follows that also

$$
\sum_{s_{1}^{\prime} \in S_{1}(m)} \mu_{21}\left(h_{0}\right)\left(s_{1}^{\prime}\right)=\sum_{s_{1}^{\prime} \in S_{1}(m)} \sigma_{1}\left(s_{1}^{\prime}\right)>0 .
$$


Hence, using the fact that $c$ satisfies Bayesian updating to get the second equality,

$$
\begin{aligned}
\beta(t \mid m) & =\frac{\sum_{s_{1}: s_{1}(t)=m} p(t) \cdot \mu_{21}(m)\left(s_{1}\right)}{\sum_{t^{\prime} \in T} \sum_{s_{1}: s_{1}\left(t^{\prime}\right)=m} p\left(t^{\prime}\right) \cdot \mu_{21}(m)\left(s_{1}\right)} \\
& =\frac{\sum_{s_{1}: s_{1}(t)=m} p(t) \cdot \mu_{21}\left(h_{0}\right)\left(s_{1}\right)}{\sum_{t^{\prime} \in T} \sum_{s_{1}: s_{1}(t)=m} p\left(t^{\prime}\right) \cdot \mu_{21}\left(h_{0}\right)\left(s_{1}\right)} \\
& =\frac{\sum_{s_{1}: s_{1}(t)=m} p(t) \cdot \sigma_{1}\left(s_{1}\right)}{\sum_{t^{\prime} \in T} \sum_{s_{1}: s_{1}(t)=m} p\left(t^{\prime}\right) \cdot \sigma_{1}\left(s_{1}\right)} \\
& =\frac{p(t) \cdot \sigma_{1}(m \mid t)}{\sum_{t^{\prime} \in T} p\left(t^{\prime}\right) \cdot \sigma_{1}\left(m \mid t^{\prime}\right)}
\end{aligned}
$$

which shows that $\beta$ is Bayesian consistent with $\sigma_{1}$.

Example 2.11 To illustrate Theorem 2.10, consider again the game in Figure 2 and the preference conjecture equilibrium c discussed in Example 3.3. The sequential equilibrium $\left(\sigma_{1}, \sigma_{2}, \beta\right)$ induced by this preference conjecture equilibrium has $\sigma_{1}=(l, m), \sigma_{2}=(a, a, a), \beta(k)=(1,0), \beta(l)=(1,0)$ and $\beta(m)=(0,1)$.

Conversely, for every sequential equilibriurn there is indeed a preference conjecture equilibrium that induces this equilibrium as the next theorem states. However, since we will prove a somewhat stronger result in the second part of Theorem 2.17, we postpone the proof to the next section (for the sake of both completeness and clarity a proof of Theorem 2.12 is given in section 2.6).

Theorem 2.12 Suppose that the assessment $\left(\sigma_{1}, \sigma_{2}, \beta\right)$ is a sequential equilibrium of a signaling game $\mathcal{S}=\left(T, M, A, p, u_{1}, u_{2}\right)$. Then there exists a preference conjecture equilibrium $\left(\mu_{12}, u_{12},\left(\mu_{21}(m), u_{21}(m)\right)_{m \in M^{*}}\right)$ with $u_{21}\left(h_{0}\right)=u_{1}$ and $u_{12}=u_{2}$ that induces $\left(\sigma_{1}, \sigma_{2}, \beta\right)$.

Corollary 2.13 Let $\mathcal{S}=\left(T, M, A, p, u_{1}, u_{2}\right)$ be a signaling game. Then there exists a preference conjecture equilibrium.

\subsection{Minimum revision equilibrium}

In a preference conjecture equilibrium player 2 can revise his conjecture about the utilities and strategies of player 1. Intuitively, one would like to keep these revisions as limited as possible. In this section we propose a way to measure this, and we study the resulting refinement of preference conjecture equilibrium and the associated refinement of sequential equilibrium. 
The measure which we are going to use is based on the number of 'utility changes' player 2 has to make in order to rationalize player 1's move. Before elaborating on this we need some notations and definitions.

$A$ (weak) ordering on a finite set $E$ is a complete and transitive binary relation on $E$. Generically, for an ordering $R$ we denote by $P$ and $I$ its asymmetric and symmetric parts, respectively. For $x, y \in E$, the expressions $x R y, x P y$, and $x I y$, are interpreted as ' $x$ is weakly preferred to $y$ ', ' $x$ is strictly preferred to $y$ ', and 'r is equivalent to $y$ '. Let $R$ and $R^{\prime}$ be two orderings. We define

$$
d(x, y):=\left\{\begin{array}{cc}
1 & \text { if } x P y \text { and not } x P^{\prime} y \\
1 & \text { if } x I y \text { and not } x I^{\prime} y \\
1 & \text { if } y P x \text { and not } y P^{\prime} x \\
0 & \text { else }
\end{array}\right.
$$

We define the distance between $R$ and $R^{\prime}$ as

$$
d\left(R, R^{\prime}\right):=\frac{1}{2} \sum_{x \in E} \sum_{y \in E} d(x, y) .
$$

Thus, the distance between two orderings is defined as the number of pairs of elements that are ranked differently in the two orderings.

\subsubsection{Minimum revision equilibrium}

Now consider a conjecture profile $c=\left(\mu_{12}, u_{12},\left(\mu_{21}(m), u_{21}(m)\right)_{m \in M^{*}}\right)$. In this profile the conjecture of player 2 in information set $m$ regarding the expected utility function of player 1's type $t$ is given by

$$
U_{21}(m)\left(t, m^{\prime}, \mu_{12}\right):=\sum_{s_{2} \in S_{2}} \mu_{12}\left(s_{2}\right) \cdot u_{21}(m)\left(t, m^{\prime}, s_{2}\left(m^{\prime}\right)\right) .
$$

The preference ordering $R_{m}^{t}$ on $M$ for type $t$ is defined by, for all $k, l \in M$

$$
k R_{m}^{t} l \quad \text { if and only if } U_{21}(m)\left(t, k, \mu_{12}\right) \geq U_{21}(m)\left(t, l, \mu_{12}\right) .
$$

Now, the distance $d\left(R_{h_{0}}^{t}, R_{m}^{t}\right)$ between the orderings $R_{m}^{t}$ and $R_{h_{0}}^{t}$ counts the number of 'utility changes' player 2 makes for type $t$ of player 1 if he observes message $m$. Based on this, we define the revision index of the conjecture profile
$c$ by

$$
d(c):=\sum_{m \in M} \sum_{t \in T} d\left(R_{h_{0}}^{t}, R_{m}^{t}\right)
$$

Example 2.14 Consider again the signaling game of Example 2.2, see Figure 2. Let $c$ be the conjecture profile such that $\mu_{12}=(a, b, b)$ and $u_{12}$ is equal to the depicted utility function. Furthermore, $\mu_{21}\left(h_{0}\right)=\mu_{21}(k)=\mu_{21}(m)=(k, m)$ and $\mu_{21}(l)=(k, l)$. Finally, $u_{21}\left(h_{0}\right), u_{21}(k)$ and $u_{21}(m)$ are equal to the depicted utility function, $u_{21}(l)\left(t_{2}, l, b\right)=4, u_{21}(l)\left(t_{2}, k, a\right)=3$ and $u_{21}(l)\left(t, m^{\prime}, a^{\prime}\right)=$ $u_{21}\left(h_{0}\right)\left(t, m^{\prime}, a^{\prime}\right)$ otherwise. Then, $R_{h_{0}}^{t_{2}}$ orders $m$ strictly above $l$ and $l$ strictly above $k ;$ and $R_{l}^{t_{2}}$ orders $l$ strictly above $k$ and $k$ strictly above $m$. Hence,
$d\left(R_{h_{11}}^{t_{2}}, R_{l}^{t_{2}}\right)=2$. 
By requiring that the revision index be as small as possible, we obtain the announced refinement of preference conjecture equilibrium.

Definition 2.15 Let c be a preference conjecture equilibrium with $u_{12}=u_{2}$ and $u_{21}\left(h_{0}\right)=u_{1}$. Then $c$ is a minimum revision equilibrium if

$$
d(c) \leq d\left(c^{\prime}\right)
$$

for all preference conjecture equilibria $c^{\prime}$ with $u_{12}^{\prime}=u_{2}$ and $u_{21}^{\prime}\left(h_{0}\right)=u_{1}$.

Since a preference conjecture equilibrium always exists, by Corollary 2.13, it follows that a minimum revision equilibrium always exists.

\subsubsection{Relation with sequential equilibrium}

Since any preference conjecture equilibrium induces a sequential equilibrium by Theorem 2.10, the concept of minimum revision equilibrium can be used to obtain a refinement of sequential equilibrium. This works as follows. The expected utility for player 1 if his type is $t$, he chooses message $m$, and player 2 plays $\sigma_{2}$ is given by

$$
U_{1}\left(t, m, \sigma_{2}\right):=\sum_{s_{2} \in S_{2}} \sigma_{2}\left(s_{2}\right) \cdot u_{1}\left(t, m, s_{2}(m)\right)
$$

Now, for a sequential equilibrium $\left(\sigma_{1}, \sigma_{2}, \beta\right)$ of $\mathcal{S}$, let $R\left(\sigma_{1}, \sigma_{2}, \beta\right)$ denote set of triples $\left(t, m, m^{\prime}\right)$ in $T \times M \times M$ for which

$$
\beta(t \mid m)>0 \text { and } U_{1}\left(t, m, \sigma_{2}\right)<U_{1}\left(t, m^{\prime}, \sigma_{2}\right) .
$$

Definition 2.16 The revision index of $\left(\sigma_{1}, \sigma_{2}, \beta\right)$ is defined as the number $\left|R\left(\sigma_{1}, \sigma_{2}, \beta\right)\right|$ of clements of the set $R\left(\sigma_{1}, \sigma_{2}, \beta\right)$. It is denoted by $r\left(\sigma_{1}, \sigma_{2}, \beta\right)$.

If a triple $\left(t, m, m^{\prime}\right)$ is in $R\left(\sigma_{1}, \sigma_{2}, \beta\right)$, then player 2 believes that type $t$ has positive probability although the observed message $m$ is inferior to $m^{\prime}$ for this type. If player 2 believes that player 1 is rational, then he should make some 'revision' in order to rationalize this. This explains the term 'revision index' in Definition 2.16. The following theorem justifies the use of this particular expression.

Theorem 2.17 Let $\mathcal{S}=\left(T, M, A, p, u_{1}, u_{2}\right)$ be a signaling game and let $\left(\sigma_{1}, \sigma_{2}, \beta\right)$ be a sequential equilibrium in $\mathcal{S}$. Then:

(a) if $c$ is a preference conjecture equilibrium with $u_{12}=u_{2}$ and $u_{21}\left(h_{0}\right)=u_{1}$ that induces $\left(\sigma_{1}, \sigma_{2}, \beta\right)$, then $d(c) \geq r\left(\sigma_{1}, \sigma_{2}, \beta\right)$.

(b) there exists a preference conjecture equilibrium $c$ with $u_{12}=u_{2}$ and with $u_{21}\left(h_{0}\right)=u_{1}$ that induces $\left(\sigma_{1}, \sigma_{2}, \beta\right)$, such that $d(c)=r\left(\sigma_{1}, \sigma_{2}, \beta\right)$. 
Proof. (a) Let $c$ be a preference conjecture equilibrium with $u_{12}=u_{2}$ and $u_{21}\left(h_{0}\right)=u_{1}$ which induces $\left(\sigma_{1}, \sigma_{2}, \beta\right)$. Take an element $\left(t, m, m^{\prime}\right)$ in $R\left(\sigma_{1}, \sigma_{2}, \beta\right)$. It suffices to show that $m R_{m}^{t} m^{\prime}$ and $m^{\prime} P_{h_{0}}^{t} m$ because this impliess that

$$
d(c)=\sum_{m \in M} \sum_{t \in T} d\left(R_{h_{0}}^{t}, R_{m}^{t}\right) \geq\left|R\left(\sigma_{1}, \sigma_{2}, \beta\right)\right|=r\left(\sigma_{1}, \sigma_{2}, \beta\right) .
$$

In rorler to show that $m R_{m}^{t} m^{\prime}$, note that $\beta(t \mid m)>0$ because $\left(t, m, m^{\prime}\right)$ is an Mment of $R\left(\sigma_{1}, \sigma_{2}, \beta\right)$. So, by $(2.3)$, there exists an $s_{1} \in S_{1}$ such that $s_{1}(t)=m$ and $\mu_{21}(m)\left(s_{1}\right)>0$. Since $c$ is a preference conjecture equilibrium, this implies that

$$
U_{21}(m)\left(t, m, \mu_{12}\right) \geq U_{21}(m)\left(t, m^{\prime}, \mu_{12}\right)
$$

and honce $R_{m}^{t}$ orders $m$ weakly above $m^{\prime}$. On the other hand, $U_{1}\left(t, m, \sigma_{2}\right)<$ $U_{1}\left(t, m^{\prime}, \sigma_{2}\right)$ since $\left(t, m, m^{\prime}\right)$ is an element of $R\left(\sigma_{1}, \sigma_{2}, \beta\right)$. This is equivalent to

$$
U_{21}\left(h_{0}\right)\left(t, m, \mu_{12}\right)<U_{21}\left(h_{0}\right)\left(t, m^{\prime}, \mu_{12}\right)
$$

and hence $R_{h_{0}}^{t}$ orders $m$ strictly below $m^{\prime}$.

(b) Let $\left(\sigma_{1}, \sigma_{2}, \beta\right)$ be a sequential equilibrium. We define the conjecture profile

$$
c^{*}=\left(\mu_{12}^{*}, u_{12}^{*},\left(\mu_{21}^{*}(m), u_{21}^{*}(m)\right)_{m \in M^{*}}\right)
$$

as follows. For player 1 we define $\mu_{12}^{*}:=\sigma_{2}$ and $u_{12}^{*}:=u_{2}$. For player 2 , we take $\mu_{21}^{*}\left(h_{0}\right):=\sigma_{1}$ and $u_{21}^{*}\left(h_{0}\right):=u_{1}$ at the start of the game. Further, for any other information set $m \in M$, we define $u_{21}^{*}(m)$ by

$$
u_{21}^{*}(m)(t, l, a):=\left\{\begin{array}{cc}
\max _{m^{\prime} \in M} u_{1}\left(t, m^{\prime}, a\right)+1 & \text { if } l=m \text { and } \\
& \text { there exists an } m^{\prime} \in M \\
u_{1}(t, l, a) & \text { s.t. }\left(t, m, m^{\prime}\right) \in R\left(\sigma_{1}, \sigma_{2}, \beta\right)
\end{array}\right.
$$

In the definition of $\mu_{21}^{*}(m)$ we distinguish two cases.

Case 1. Suppose there exists an $s_{1} \in S_{1}(m)$ such that $\sigma_{1}\left(s_{1}\right)>0$. Then we define $\mu_{21}^{*}(m)$ for each $s_{1} \in S_{1}(m)$ by

$$
\mu_{21}^{*}(m)\left(s_{1}\right):=\frac{\sigma_{1}\left(s_{1}\right)}{\sum_{s_{1}^{\prime} \in S_{1}(m)} \sigma_{1}\left(s_{1}^{\prime}\right)} .
$$

Case 2. Suppose that $\sigma_{1}\left(s_{1}\right)=0$ for all $s_{1} \in S_{1}(m)$. Take a pure strategy $s_{1}^{*}$ with $\sigma_{1}\left(s_{1}^{*}\right)>0$. Then we know that $s_{1}^{*}(t) \neq m$ for each type $t$. Now define for type $t \in T$ the pure strategy $s_{1}^{t, m}$ by

$$
s_{1}^{t, m}\left(t^{\prime}\right):=\left\{\begin{array}{cc}
m & \text { if } t^{\prime}=t \\
s_{1}^{*}\left(t^{\prime}\right) & \text { if } t^{\prime} \neq t
\end{array}\right.
$$

Clearly all strategies $s_{1}^{t, m}$ are elements of $S_{1}(m)$ and $s_{1}^{t, m} \neq s_{1}^{t^{\prime}, m}$ whenever $t \neq t^{\prime}$. Thus we can define for each pure strategy $s_{1} \in S_{1}(m)$

$$
\mu_{21}(m)\left(s_{1}\right):=\left\{\begin{array}{cl}
\frac{\beta(t) m)}{r^{\prime}(t)} & \text { if } s_{1}=s_{1}^{t, m} \\
0 & \text { if } s_{1} \neq s_{1}^{t, m}
\end{array}\right.
$$


Next define $\mu_{21}^{*}(m)$ by, for all pure strategies $s_{1} \in S_{1}(m)$,

$$
\mu_{21}^{*}(m)\left(s_{1}\right):=\frac{\mu_{21}(m)\left(s_{1}\right)}{\sum_{s_{1}^{\prime} \in S_{1}(m)} \mu_{21}(m)\left(s_{1}^{\prime}\right)} .
$$

The denominator is not equal to zero, because there is at least one type $t$ with $\beta(t \mid m)>0$, and for this type $\mu_{21}(m)\left(s_{1}^{t, m}\right)>0$ while indeed $s^{t, m}$ is an element of $S_{1}(m)$.

We show that $c^{*}$ is a preference conjecture equilibrium with $d\left(c^{*}\right)=r\left(\sigma_{1}, \sigma_{2}, \beta\right)$ that induces $\left(\sigma_{1}, \sigma_{2}, \beta\right)$.

First we show that $c^{*}$ induces $\left(\sigma_{1}, \sigma_{2}, \beta\right)$. We only have to prove (2.3). Let $t \in T$ and $m \in M$. In Case 1 ,

$$
\begin{aligned}
\beta(t \mid m) & =\frac{p(t) \cdot \sigma_{1}(m \mid t)}{\sum_{t^{\prime} \in T} p\left(t^{\prime}\right) \cdot \sigma_{1}\left(m \mid t^{\prime}\right)}=\frac{p(t) \cdot \sum_{s_{1}: s_{1}(t)=m} \sigma_{1}\left(s_{1}\right)}{\sum_{t^{\prime} \in T} p\left(t^{\prime}\right) \cdot \sum_{s_{1}: s_{1}\left(t^{\prime}\right)=m} \sigma_{1}\left(s_{1}\right)} \\
& =\frac{\sum_{s_{1}: s_{1}(t)=m} p(t) \cdot \mu_{21}^{*}(m)\left(s_{1}\right)}{\sum_{t^{\prime} \in T} \sum_{s_{1}: s_{1}\left(t^{\prime}\right)=m} p\left(t^{\prime}\right) \mu_{21}^{*}(m)\left(s_{1}\right)}
\end{aligned}
$$

where the first equality follows from Bayesian consistency of $\beta$ with $\sigma_{1}$, the second one from the definition of $\sigma_{1}(m \mid t)$, and the third one from the definition of $\mu_{21}^{*}(m)$. In Case 2,

$$
\begin{aligned}
\beta(t \mid m)=\frac{\beta(t \mid m)}{\sum_{t^{\prime} \in T} \beta\left(t^{\prime} \mid m\right)} & =\frac{\sum_{s_{1}: s_{1}(t)=m} p(t) \cdot \mu_{21}(m)\left(s_{1}\right)}{\sum_{t^{\prime} \in T} \sum_{s_{1}: s_{1}\left(t^{\prime}\right)=m} p\left(t^{\prime}\right) \cdot \mu_{21}(m)\left(s_{1}\right)} \\
& =\frac{\sum_{s_{1}: s_{1}(t)=m} p(t) \cdot \mu_{21}^{*}(m)\left(s_{1}\right)}{\sum_{l^{\prime} \in T} \sum_{s_{1}: s_{1}\left(l^{\prime}\right)=m} p\left(t^{\prime}\right) \cdot \mu_{21}^{*}(m)\left(s_{1}\right)}
\end{aligned}
$$

so also in this case (2.3) holds. Hence, $c$ induces $\left(\sigma_{1}, \sigma_{2}, \beta\right)$.

Next we show that $d\left(c^{*}\right)=r\left(\sigma_{1}, \sigma_{2}, \beta\right)$. Let $t \in T$ and $m \in M$. If there is no $m^{\prime}$ with $\left(t, m, m^{\prime}\right) \in R\left(\sigma_{1}, \sigma_{2}, \beta\right)$, then $u_{21}^{*}(m)=u_{1}=u_{21}^{*}\left(h_{0}\right)$ and therefore

$$
d\left(R_{h_{0}}^{t}, R_{m}^{t}\right)=0
$$

Otherwise, consider an $m^{\prime} \in M$ with $\left(t, m, m^{\prime}\right) \in R\left(\sigma_{1}, \sigma_{2}, \beta\right)$. Then, by definition of $R\left(\sigma_{1}, \sigma_{2}, \beta\right)$, we have $\beta(t \mid m)>0$ and $U_{1}\left(t, m, \sigma_{2}\right)<U_{1}\left(t, m^{\prime}, \sigma_{2}\right)$. Hence

$$
\sum_{s_{2} \in S_{2}} \mu_{12}^{*}\left(s_{2}\right) u_{21}^{*}\left(h_{0}\right)\left(t, m, s_{2}(m)\right)<\sum_{s_{2} \in S_{2}} \mu_{12}^{*}\left(s_{2}\right) u_{21}^{*}\left(h_{0}\right)\left(t, m^{\prime}, s_{2}\left(m^{\prime}\right)\right)
$$


and $R_{h_{0}}^{t}$ orders $m$ strictly below $m^{\prime}$. However, notice that in this case

$$
u_{21}^{*}(m)(t, m, a):=\max _{m^{\prime \prime} \in M} u_{1}\left(t, m^{\prime \prime}, a\right)+1
$$

and $u_{21}^{*}(m)\left(t, m^{\prime}, a\right)=u_{1}\left(t, m^{\prime}, a\right)$ for all $a \in A$. Hence, $R_{m}^{t}$ orders $m$ strictly above $m^{\prime}$ and it orders any two messages different from $m$ in the same way as $R_{h_{0}}^{t}$. Thus, since $\beta(t \mid m)>0$ in this case, we obtain:

$$
d\left(R_{h_{0}}^{t}, R_{m}^{t}\right)=\left|\left\{m^{\prime} \in M \mid U_{1}\left(t, m, \sigma_{2}\right)<U_{1}\left(t, m^{\prime}, \sigma_{2}\right)\right\}\right| .
$$

Summation over all messages $m$ and all types $t$ yields

$$
\begin{aligned}
r\left(\sigma_{1}, \sigma_{2}, \beta\right) & =\sum_{(t, m): \beta(t \mid m)>0}\left|\left\{m^{\prime} \in M \mid U_{1}\left(t, m, \sigma_{2}\right)<U_{1}\left(t, m^{\prime}, \sigma_{2}\right)\right\}\right| \\
& =\sum_{(t, m): \beta(t \mid m)>0} d\left(R_{h_{0}}^{t}, R_{m}^{l}\right)=d(c)
\end{aligned}
$$

where the last equality follows from the fact that $d\left(R_{h_{0}}^{t}, R_{m}^{t}\right)=0$ whenever $\beta(t \mid m)=0$.

Finally we show that $c^{*}$ is a preference conjecture equilibrium. First note that $\mu_{21}^{*}\left(h_{0}\right)=\sigma_{1}$. So, for $m \in M$ such that $\sum_{s_{1}^{\prime} \in S_{1}(m)} \mu_{21}^{*}\left(h_{0}\right)\left(s_{1}^{\prime}\right)>0$, the conjecture $\mu_{21}^{*}(m)$ is defined by Case 1, and hence $c^{*}$ satisfies Bayesian updating. Secondly, we show optimality for player 2. Take an $m \in M$ and an $s_{2} \in S_{2}$ with $\mu_{12}^{*}\left(s_{2}\right)>0$. Since $\sigma_{2}\left(s_{2}\right)=\mu_{12}^{*}\left(s_{2}\right)>0$, optimality of $\sigma_{2}$ with respect to $\beta$ implies that

$$
U_{2}\left(m, s_{2}, \beta\right) \geq U_{2}\left(m, s_{2}^{\prime}, \beta\right)
$$

for all $s_{2}^{\prime} \in S_{2}$. Hence, using the equality

$$
U_{2}\left(m, s_{2}, \beta\right)=\sum_{t \in T} \beta(t \mid m) \cdot u_{2}\left(t, m, s_{2}(m)\right)
$$

together with (2.3) and $u_{12}^{*}=u_{2}$, we see for all $s_{2}^{\prime} \in S_{2}$ that

$$
\begin{aligned}
U_{12}^{*}\left(m, \mu_{21}^{*}(m), s_{2}\right) & =\sum_{t \in T} p(t) \sum_{s_{1}: s_{1}(t)=m} \mu_{21}^{*}(m)\left(s_{1}\right) \cdot u_{12}^{*}\left(t, m, s_{2}(m)\right) \\
& \geq \sum_{t \in T} p(t) \sum_{s_{1}: s_{1}(t)=m} \mu_{21}^{*}(m)\left(s_{1}\right) \cdot u_{12}^{*}\left(t, m, s_{2}^{\prime}(m)\right) \\
& =U_{12}^{*}\left(m, \mu_{21}^{*}(m), s_{2}^{\prime}\right)
\end{aligned}
$$

Hence, $s_{2}$ is optimal for player 2 with respect to $\mu_{21}^{*}(m)$ and $u_{12}^{*}$.

Thirdly we show optimality for player 1 . Let $s_{1} \in S_{1}$. If $\mu_{21}^{*}\left(h_{0}\right)\left(s_{1}\right)>0$ then $\sigma_{1}\left(s_{1}\right)>0$, so optimality of $\sigma_{1}$ with respect to $\sigma_{2}$ yields that $s_{1}$ is optimal with respect to $\sigma_{2}$ in the game $\mathcal{S}$. Hence $s_{1}$ is optimal with respect to $\mu_{12}^{*}=\sigma_{2}$
and $u_{21}^{*}\left(h_{0}\right)=u_{1}$. 
Take an $m \in M$ and assume that $\mu_{21}^{*}(m)\left(s_{1}\right)>0$. We will show that $s_{1}$ is optimal for player 1 with respect to $\mu_{12}^{*}$ and $u_{21}^{*}(m)$.

First consider Case 1 in the definition of $\mu_{21}^{*}(m)$. Clearly $\sigma_{1}\left(s_{1}\right)>0$ in this case. So, we at least know that $s_{1}$ must be optimal with respect to $\sigma_{2}$ and $u_{1}$. Since $\mu_{12}^{*}:=\sigma_{2}$, it remains to show that $u_{21}^{*}(m)=u_{1}$.

Since we are in Case 1 we know that there exists an $s_{1}^{\prime} \in S_{1}(m)$ such that $\sigma_{1}\left(s_{1}^{\prime}\right)>0$. This implies that $m$ is played with positive probability under $\sigma_{1}$. Then the optimality of $\sigma_{1}$ with respect to $\sigma_{2}$ implies that the set

$$
\left\{\left(t, m^{\prime}\right) \in T \times M \mid \beta(t \mid m)>0 \text { and } U_{1}\left(t, m, \sigma_{2}\right)<U_{1}\left(t, m^{\prime}, \sigma_{2}\right)\right\}
$$

is empty. So, there is no $\left(t, m^{\prime}\right)$ such that $\left(t, m, m^{\prime}\right) \in R\left(\sigma_{1}, \sigma_{2}, \beta\right)$. Hence, $u_{21}^{*}(m)=u_{1}$.

Next consider Case 2. So, $\sigma_{1}\left(s_{1}^{\prime}\right)=0$ for all $s_{1}^{\prime} \in S_{1}(m)$. Since $\mu_{21}^{*}(m)\left(s_{1}\right)>0$, we know that in this case $s_{1}=s_{1}^{t, m}$ for a unique $t \in T$. So, $\beta(t \mid m)>0$ for this $t$, by definition of $\mu_{21}^{*}(m), \mu_{21}(m)$ and $s_{1}^{t, m}$. We distinguish two subcases.

First, suppose that message $m$ is not optimal for $t$ with respect to $\sigma_{2}$ and $u_{1}$. Then there exists an $m^{\prime} \in M$ such that $U_{1}\left(t, m, \sigma_{2}\right)<U_{\mathbf{1}}\left(t, m^{\prime}, \sigma_{2}\right)$, and therefore $\left(t, m, m^{\prime}\right)$ is an element of $R\left(\sigma_{1}, \sigma_{2}, \beta\right)$. So, by definition,

$$
u_{21}^{*}(m)(t, m, a)=\max _{m^{\prime \prime} \in M} u_{1}\left(t, m^{\prime \prime}, a\right)+1>u_{1}\left(t, m^{\prime}, a\right)=u_{21}^{*}(m)\left(t, m^{\prime}, a\right)
$$

for all $a$ and $m^{\prime} \neq m$. Thus, $s_{1}(t)=s_{1}^{l, m}(t)=m$ is optimal for type $t$ w.r.t $u_{21}^{*}(m)$, even regardless of what $\mu_{12}^{*}$ is. Next, consider any other $t^{\prime} \neq t$. By the choice of $s_{1}^{*}\left(t^{\prime}\right)$ in the definition of $s_{1}^{t, m}$, message $s_{1}\left(t^{\prime}\right)=s_{1}^{t, m}\left(t^{\prime}\right)=s_{1}^{*}\left(t^{\prime}\right)$ is optimal for $t^{\prime}$ w.r.t. $\sigma_{2}$ and $u_{1}$. Since $t^{\prime} \neq t$, we have $u_{21}^{*}(m)=u_{1}$, and $s_{1}\left(t^{\prime}\right)$ is also optimal w.r.t. $\mu_{12}^{*}$ and $u_{21}^{*}(m)$.

Secondly, suppose that $m$ is optimal for $t$ with respect to $\sigma_{2}$ and $u_{1}$. Then there exists no $m^{\prime}$ such that $\left(t, m, m^{\prime}\right) \in R\left(\sigma_{1}, \sigma_{2}, \beta\right)$. Hence, $u_{21}^{*}(m)=u_{1}$, and $s_{1}(t)=s_{1}^{t, m}(t)=m$ is optimal w.r.t. $\mu_{12}^{*}$ and $u_{21}^{*}(m)$. The argument for types $t^{\prime} \neq t$ is identical to the previous case.

We conclude this section with a few direct consequences of Theorem 2.17.

Definition 2.18 A sequential equilibrium $\left(\sigma_{1}, \sigma_{2}, \beta\right)$ in a signaling game $\mathcal{S}=$ $\left(T, M, A, p, u_{1}, u_{2}\right)$ is called a minimum revision sequential equilibrium if it minimizes the revision index among all sequential equilibria of the game.

We immediately have the following corollary.

Corollary 2.19 If $c$ is a minimum revision equilibrium in $\mathcal{S}$, then the induced sequential equilibrium is a minimum revision sequential equilibrium. Conversely, for every minimum revision sequential equilibrium $\left(\sigma_{1}, \sigma_{2}, \beta\right)$ there exists a minimum revision equilibrium $c$ with $u_{21}\left(h_{0}\right)=u_{1}$ and $u_{12}=u_{2}$ that induces $\left(\sigma_{1}, \sigma_{2}, \beta\right)$ 
Further, in the special case where under $\sigma_{1}$ every information set of player 2 is reached with positive probability, the set $R\left(\sigma_{1}, \sigma_{2}, \beta\right)$ will be empty. Hence, $\left(\sigma_{1}, \sigma_{2}, \beta\right)$ is a minimum revision sequential equilibrium with revision index 0 , and every minimum revision equilibrium with $u_{21}\left(h_{0}\right)=u_{1}$ and $u_{12}=u_{2}$ has revision index 0 .

\subsection{Examples and comparison to the intuitive criterion}

A commonly used refinement of sequential equilibrium in signaling games is the intuitive criterion (see [Cho \& Kreps, 1987]). This criterion restricts possible beliefs on information sets that are not reached with positive probability in equilibrium, as follows. Suppose a message $m$ is sent that has zero probability in equilibrium. Then we ask which type could possibly benefit from sending $m$, in the sense that there exists an action $a \in A$ such that

(i) $u_{1}(t, m, a) \geq \sum_{s_{1} \in S_{1}} \sigma_{1}\left(s_{1}\right) \sum_{s_{2} \in S_{2}} \sigma_{2}\left(s_{2}\right) u_{1}\left(t, s_{1}(t), s_{2}\left(s_{1}(t)\right)\right)$, and

(ii) there is $\beta(\cdot \mid m) \in \Delta(T)$ such that $a \in \arg \max _{a^{\prime} \in A} \sum_{t^{\prime} \in T} \beta\left(t^{\prime} \mid m\right) u_{2}\left(t^{\prime}, m, a^{\prime}\right)$.

Condition (i) states that if $m$ is followed by $a$, then the utility to type $t$ is at least as high as the equilibrium utility for this type. Condition (ii) states that $a$ is a potential optimal action for player 2 after $m$.

A sequential equilibrium $\left(\sigma_{1}, \sigma_{2}, \beta\right)$ is said to survive the intuitive criterion if for every $m$ such that (1) $\sigma_{1}\left(s_{1}\right)=0$ for all $s_{1} \in S_{1}(m)$ and (2) there are types $t$ for which an $a \in A$ satisfying (i) and (ii) above exists, we have $\beta(t \mid m)>0$ only if $t$ is a type as in (2).

In this section we consider two examples of signaling games. In both examples we compute all sequential equilibria and thus, basically, all preference conjecture equilibria with the utility functions of the signaling game conjectured at the beginning (cf. Theorems 2.10 and 2.12). We also compare the refinements induced by minimum revision and intuitive criterion. In the first example there are minimum revision equilibria that survive the intuitive criterion and others that don't. The second example shows that the set of minimum revision equilibria and the set of equilibria surviving the intuitive criterion can be disjoint.

Example 2.20 See Figure 2.1 for the extensive form of the game and the description of all sequential equilibria. For convenience, the equilibria are described in terms of behavioral strategies. E.g., $(b, \lambda a+(1-\lambda) b)$ is the behavioral strategy where player 2 plays $b$ if player 1 plays $A$, and a with probability $\lambda$ and $b$ with probability $1-\lambda$ if player 1 plays $B$. Further, $\alpha$ is the belief of player 2 that player has type $t_{1}$ if he plays $A$, and $\beta$ is the belief of player 2 that player 1 has type $t_{1}$ if he plays $B$. The revision index is denoted by $r$. 


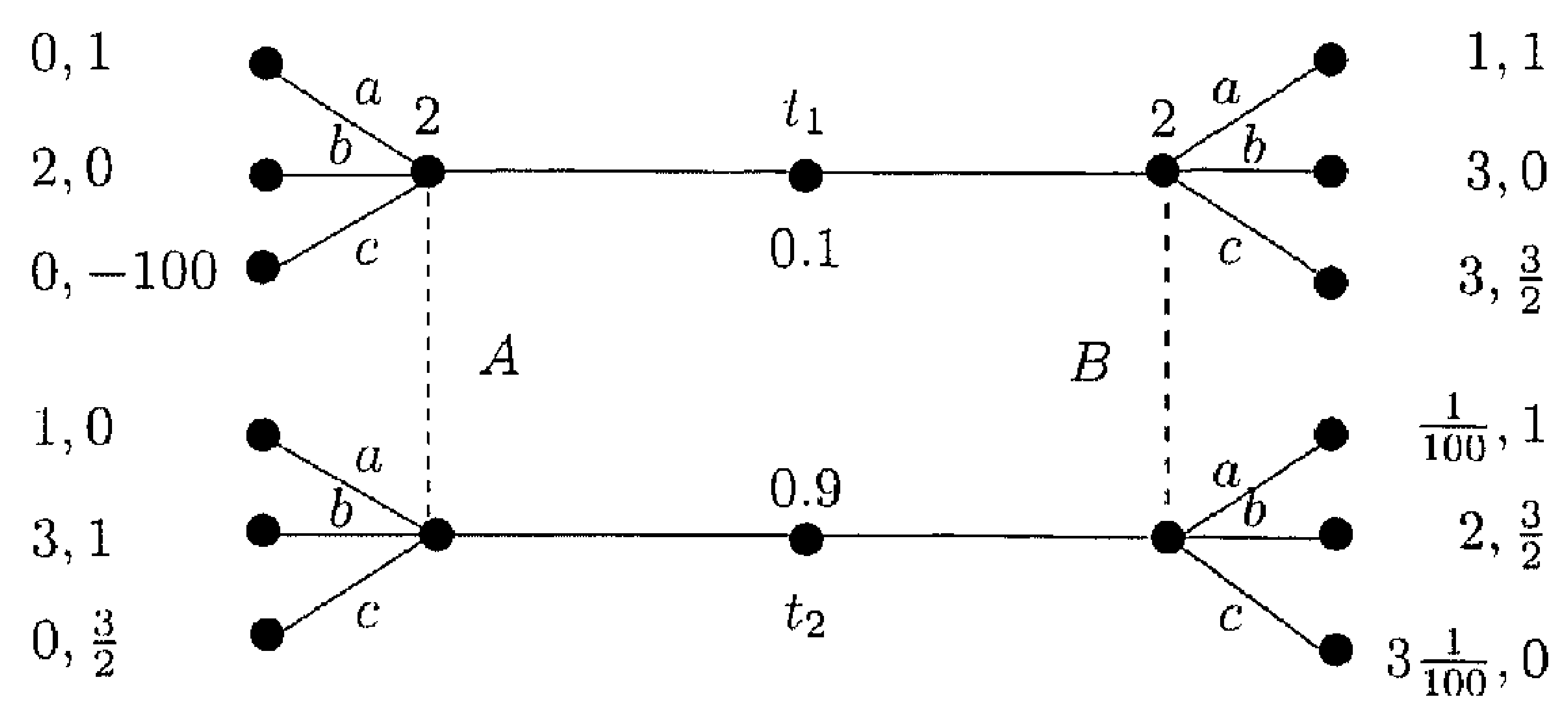

\begin{tabular}{|l|c|c|c|c|}
\hline Equilibrium (in behavioural strategies) & $\alpha$ & $\beta$ & $r$ & intuitive \\
\hline$\left\{(A, A),(b, \lambda a+(1-\lambda) b) ; \frac{1}{2}<\lambda \leq 1\right\}$ & 0.1 & $\frac{1}{3}$ & 2 & yes \\
$\left\{(A, A),\left(b, \frac{1}{2} a+\frac{1}{2} b\right)\right\}$ & 0.1 & $\frac{1}{3}$ & 1 & yes \\
$\{(A, A),(b, a)\}$ & 0.1 & $\left(\frac{1}{3}, \frac{2}{3}\right)$ & 2 & yes \\
$\left\{(A, A),(b, \lambda a+(1-\lambda) c) ; \frac{1}{2}<\lambda \leq 1\right\}$ & 0.1 & $\frac{2}{3}$ & 2 & yes \\
$\left\{(A, A),\left(b, \frac{1}{2} a+\frac{1}{2} c\right)\right\}$ & 0.1 & $\frac{2}{3}$ & 1 & yes \\
$\{(B, B),(c, b)\}$ & 0 & 0.1 & 1 & yes \\
$\{(B, B),(c, b)\}$ & $\left(0, \frac{1}{201}\right)$ & 0.1 & 2 & no \\
$\left\{(B, B),(\lambda b+(1-\lambda) c, b) ; 0<\lambda<\frac{2}{3}\right\}$ & $\frac{1}{201}$ & 0.1 & 2 & no \\
$\left\{(B, B),\left(\frac{2}{3} b+\frac{1}{3} c, b\right)\right\}$ & $\frac{1}{201}$ & 0.1 & 1 & no \\
$\{(B, B),(a, b)\}$ & 1 & 0.1 & 1 & no \\
$\{(B, B),(a, b)\}$ & $\left(\frac{1}{2}, 1\right)$ & 0.1 & 2 & no \\
$\left\{(B, B),(\lambda a+(1-\lambda) b, b) ; 0<\lambda<\frac{1}{2}\right\}$ & $\frac{1}{2}$ & 0.1 & 2 & no \\
$\left\{(B, B),\left(\frac{1}{2} a+\frac{1}{2} b, b\right)\right\}$ & $\frac{1}{2}$ & 0.1 & 1 & no \\
\hline
\end{tabular}

Figure 2.1: Example 2.20 


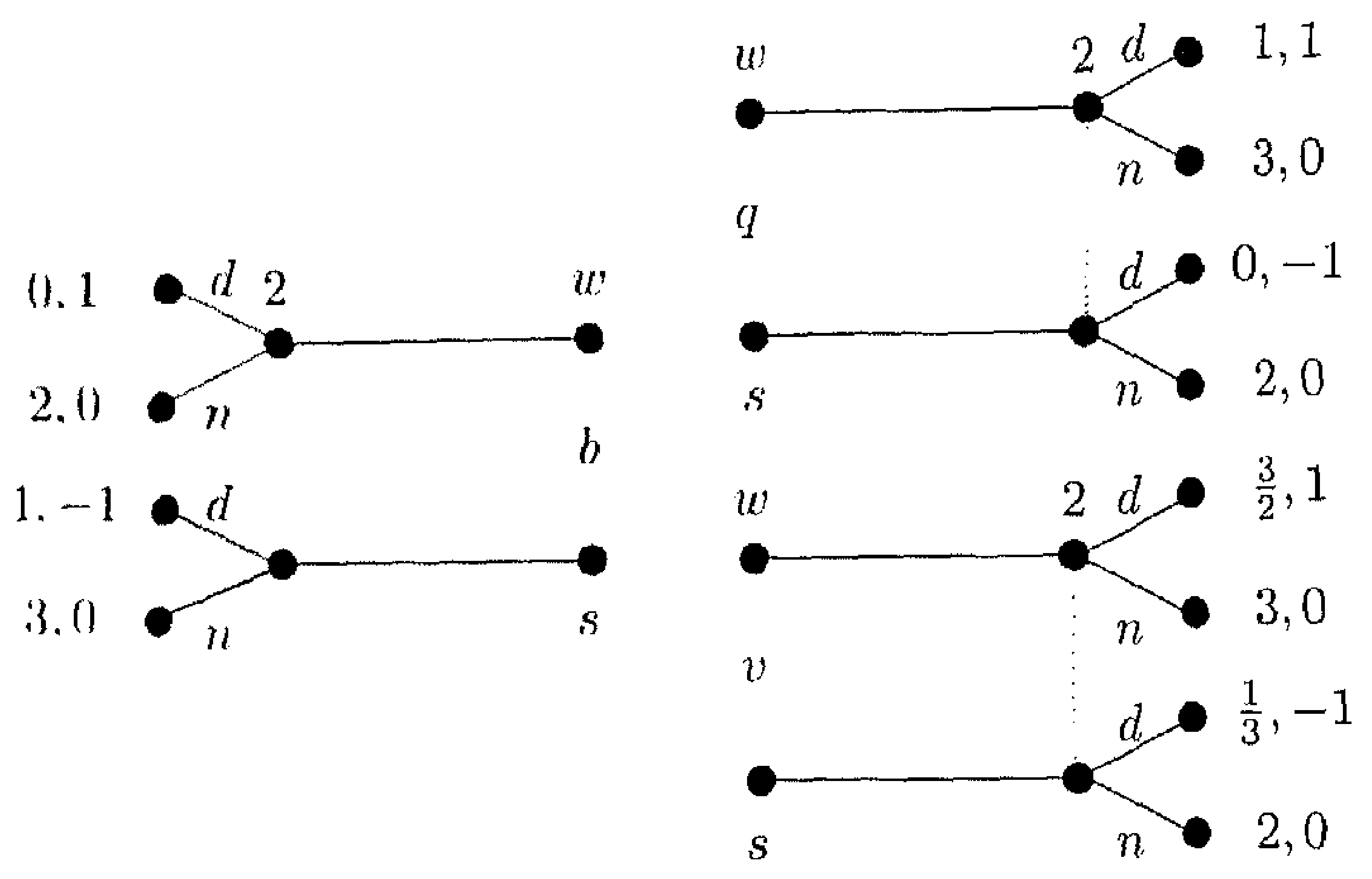

Figure 2.2: Example 2.21

Example 2.21 This example shows that the set of minimum revision sequential equilibria and the set of sequential equilibria that survive the intuitive criterion can be disjoint. Consider the game in Figure 2.2, a game inspired by the beer and quiche game of Cho and Kreps (1987). Player 1 has the extra option of vegetarian quiche, which contains enough spinach for him to be stronger in case of a duel. Player 1 has two types: weak (w) and strong ( $s$ ), with the weak type occurring with probability $\frac{1}{10}$; and three messages: beer $(b)$, quiche $(q)$ and vegetarian quiche (v). Player 2 has two actions: duel (d) and not duel ( $n$ ).

It can be shown that the only sequential equilibrium in this game that survives the intuitive criterion is the one where both types of player 1 drink beer, and player does not duel if and only if player 1 drinks beer, and believes that player 1 is weak with probability one if he eats quiche or vegetarian quiche. So this is similar to the original beer and quiche game. This equilibrium has revision index equal to 2 and is not a minimal revision equilibrium: there are sequential equilibria that have (the minimal) revision index 1 , for instance the equilibrium where both types of player 1 eat quiche (q), player 2 duels if and only if player 1 drinks beer, believes that beer signals the weak type with probability one and vegetarian quiche signals the strong type with probability greater than $1 / 2$.

\subsection{Structure and computation}

In this section we give a representation of the set of sequential equilibria of a signaling game as a union of subsets on which the revision index is constant. Since these subsets are polyhedral, given by systems of linear inequalities, this representation is convenient for computational purposes.

Lat $I \subseteq S_{1}$ be a subset of pure strategies for player 1 and $J \subseteq S_{2}$ a subset of 
pure strategies for player 2. Define

$$
\begin{aligned}
U(J):=\left\{\sigma_{1} \in\right. & \Delta\left(S_{1}\right) \mid \\
& \text { all } \left.\sigma_{2} \in \Delta(J) \text { are optimal with respect to } \sigma_{1}\right\}
\end{aligned}
$$

and

$$
\begin{aligned}
U(I):=\left\{\sigma_{2} \in\right. & \Delta\left(S_{2}\right) \mid \\
& \text { all } \left.\sigma_{1} \in \Delta(I) \text { are optimal with respect to } \sigma_{2}\right\} .
\end{aligned}
$$

These sets are polytopes since all inequalities defining them are linear. As is not hard to verify (cf. [Borm et al. 2003]), the set of Nash equilibria of $\mathcal{S}$ is equal to

$$
N E(\mathcal{S})=\bigcup_{I \subseteq S_{1}, J \subseteq S_{2}}(\Delta(I) \cap U(J)) \times(\Delta(J) \cap U(I)) .
$$

The difference between sequential equilibrium and Nash equilibrium is that in a sequential equilibrium player 2 also plays optimally on information sets reached with zero probability (cf. the definition of sequential equilibrium). Define

$$
M(I):=\left\{m \in M \mid \text { for all } t \in T \text { and } s_{1} \in I: s_{1}(t) \neq m\right\}
$$

and

$$
B(I, J):=\left\{\beta \in \Delta(T)^{M(I)} \mid \text { every } s_{2} \in J\right.
$$

is optimal with respect to $\beta$ on every $m \in M(I)\}$.

Since, in a sequential equilibrium, the beliefs of player 2 on information sets that are reached with positive probability are completely determined by Bayesian consistency, in describing such an equilibrium we only have to consider beliefs on information sets that are reached with zero probability. With some abuse of notation we therefore present a sequential equilibrium $\left(\sigma_{1}, \sigma_{2}, \beta\right)$ also as $\left(\sigma_{1}, \sigma_{2},(\beta(m))_{m \in M(I)}\right)$ with $I=\left\{s_{1} \in S_{1} \mid \sigma_{1}\left(s_{1}\right)>0\right\}$.

Lemma 2.22 The set of sequential equilibria of the signaling game $\mathcal{S}$ is equal to

$$
S E(\mathcal{S})=\bigcup_{I \subseteq S_{1}, J \subseteq S_{2}}(\Delta(I) \cap U(J)) \times(\Delta(J) \cap U(I)) \times B(I, J) .
$$

Proof. First let $\left(\sigma_{1}, \sigma_{2}, \beta\right)$ be an clement of

$$
\bigcup_{I \subseteq S_{1}, J \subseteq S_{2}}(\Delta(I) \cap U(J)) \times(\Delta(J) \cap U(I)) \times B(I, J) .
$$

In view of the definition of sequential equilibrium and (2.4) we still have to show that every $s_{2} \in S_{2}$ with $\sigma_{2}\left(s_{2}\right)>0$ is optimal with respect to $\beta$ on every $m \in M(I)$, but this follows immediately from $s_{2} \in J$ and the definition of $B(I, J)$. 
Conversely; let $\left(\sigma_{1}, \sigma_{2}, \beta\right)$ be a sequential equilibrium (with $\beta$ only described on information sets reached witl probability zero). Take $I:=\left\{s_{1} \in S_{1} \mid \sigma_{1}\left(s_{1}\right)>\right.$ $0\}$ and $J:=\left\{s_{2} \in S_{2} \mid \sigma_{2}\left(s_{2}\right)>0\right\}$. Then, since $\left(\sigma_{1}, \sigma_{2}\right)$ is a Nash equilibrium in $\mathcal{S}$, we have

$$
\left(\sigma_{1}, \sigma_{2}\right) \in(\Delta(I) \cap U(J)) \times(\Delta(J) \cap U(I)) .
$$

Finally, since every $s_{2} \in S_{2}$ with $\sigma_{2}\left(s_{2}\right)>0$ is optimal with respect to $\beta$ on every $m$ reached with zero probability, we have $\beta \in B(I, J)$.

We will refine the representation of the set of sequential equilibria in Lemma 2.22 based on the revision index of a sequential equilibrium. Since the revision index is related to preferences of player 1 , this means that the sets $I$ in this representation have to be refined. For every type $t \in T$, let $R^{t}$ be a (for the moment arbitrary) ordering on $M$, let $R=\left(R^{t}\right)_{t \in T}$ denote a profile of such orderings, and $\mathcal{R}$ the set of all such profiles. For a mixed strategy $\sigma_{2} \in \Delta\left(S_{2}\right)$ and type $t \in T$, define the ordering $R^{t}\left(\sigma_{2}\right)$ by

$$
m R^{t}\left(\sigma_{2}\right) m^{\prime} \Leftrightarrow \sum_{s_{2} \in S_{2}} \sigma_{2}\left(s_{2}\right) u_{1}\left(t, m, s_{2}(m)\right) \geq \sum_{s_{2} \in S_{2}} \sigma_{2}\left(s_{2}\right) u_{1}\left(t, m^{\prime}, s_{2}\left(m^{\prime}\right)\right)
$$

for all $m, m^{\prime} \in M$. (Recall that this coincides with the ordering $R_{h_{0}}^{l}$ in Section 5.) The associated profile is denoted by $R\left(\sigma_{2}\right)$.

For $R \in \mathcal{R}$ define

$$
I(R):=\left\{s_{1} \in S_{1} \mid s_{1}(t) R^{t} m \text { for all } t \in T \text { and } m \in M\right\}
$$

and

$$
U(R):=\left\{\sigma_{2} \in \Delta\left(\sigma_{2}\right) \mid R\left(\sigma_{2}\right)=R\right\} .
$$

Let $a$ be a correspondence that assigns to every $m \in M(I(R))$ a nonempty subset of $T$, and let $A(R)$ denote the set of all such correspondences. For $\beta \in B(I(R), J)$ let $\bar{\beta}$ be the correspondence that assigns to every $m \in M(I(R))$ the support of $\beta(m)$, i.e., $\bar{\beta}(m)=\{t \in T \mid \beta(t \mid m)>0\}$. Obviously, $\bar{\beta} \in A(R)$. Finally, let $B_{a}(I(R), J):=\{\beta \in B(I(R), J) \mid \bar{\beta}=a\}$ for every $a \in A(R)$.

The proof of the following lemma is left to the reader. Lemma 2.23 The set of sequential equilibria in the signaling game $\mathcal{S}$ is equal
to

$$
S E(\mathcal{S})=\bigcup_{R \in \mathcal{R}, J \subseteq S_{2}} \bigcup_{a \in A(R)}(\Delta(I(R)) \cap U(J)) \times(\Delta(J) \cap U(R)) \times B_{a}(I(R), J) .
$$

The usefulness of this representation is demonstrated by the following theorem, which states that the revision index is constant on the sets in this representation.

Theorem 2.24 In the game $\mathcal{S}$, let $R \in \mathcal{R}, a \in A(R)$, and $J \subseteq S_{2}$. Let $\left(\sigma_{1}, \sigma_{2}, \beta\right)$ and $\left(\sigma_{1}^{\prime}, \sigma_{2}^{\prime}, \beta^{\prime}\right)$ be elements of

$$
(\Delta(I(R)) \cap U(J)) \times(\Delta(J) \cap U(R)) \times B_{a}(I(R), J) .
$$

Then $r\left(\sigma_{1}, \sigma_{2}, \beta\right)=r\left(\sigma_{1}^{\prime}, \sigma_{2}^{\prime}, \beta^{\prime}\right)$. 
Proof. Suppose the two revision indices are different, say $r\left(\sigma_{1}, \sigma_{2}, \beta\right)<$ $r\left(\sigma_{1}^{\prime}, \sigma_{2}^{\prime}, \beta^{\prime}\right)$. Since $\bar{\beta}=\bar{\beta}^{\prime}=a$, by definition of the revision index there must be messages $m, \tilde{m} \in M(I(R))$ and a type $t \in a(m)$ such that $U_{1}\left(t, m, \sigma_{2}\right) \geq$ $U_{1}\left(t, \bar{m}, \sigma_{2}\right)$ and $U_{1}\left(t, m, \sigma_{2}^{\prime}\right)<U_{1}\left(t, \tilde{m}, \sigma_{2}^{\prime}\right)$. This implies $R^{t}\left(\sigma_{2}\right) \neq R_{t}\left(\sigma_{2}^{\prime}\right)$ and thus $R \neq R\left(\sigma_{2}\right)$ or $R \neq R\left(\sigma_{2}^{\prime}\right)$. Thus, either $\sigma_{2} \notin U(R)$ or $\sigma_{2}^{\prime} \notin U(R)$, a contradiction.

It follows, in particular, that the set of minimum revision equilibria is the union of sets $(\Delta(I(R)) \cap U(J)) \times(\Delta(J) \cap U(I(R))) \times B_{a}(I(R), J)$ with the same (minimal) revision index.

We conclude this section with an example.

Example 2.25 Consider Example 2.20. As an illustration of Theorem 2.24, we compute one set with constant revision index. Assume that both types strictly prefer $A$ over $B$, which means that $I(R)$ contains only one element, namely $(A, A)$. It is casily seen that this implies $U(J)=\Delta(I(R))=\{(A, A)\}$. Now we must choose $J$ such that all $\sigma_{2} \in \Delta(J)$ are optimal with respect to $(A, A)$. Thereforc we first choose

$$
J=\{(b, a),(b, b)\},
$$

that is we choose a set of strategies that can simultaneously be optimal. The set $U(R)$ contains all mixed strategies for player 2 that make player 1 willing to play $(A, A)$. Since we have to take the intersection with $\Delta(J)$ afterwards, we only consider that part of $U(R)$ that puts positive weight only on strategies in $J$. Since we want both types to strictly prefer $A$, we obtain (the behavioral strategies) $\sigma_{2}=(b, \lambda a+(1-\lambda) b)$ with $\lambda \in\left(\frac{1}{2}, 1\right]$. Given this $I(R)$, we have $M(I(R))=\{B\}$ and we choose $a(B)=\left\{t_{1}, t_{2}\right\}$. Computation of the beliefs that make all this possible yields $\beta=\frac{1}{3}$ and we have the set of sequential equilibria

$$
\left\{(A, A),(b, \lambda a+(1-\lambda) b) ; \lambda \in\left(\frac{1}{2}, 1\right] ; \beta=\frac{1}{3}\right\}
$$

with revision index $r=2$.

\subsection{Remaining proofs}

First we prove that in signaling games consistency, in the way it is defined in [Kreps \& Wilson, 1982], and Bayesian consistency are equivalent. To that purpose we first define consistency. Secondly we give a proof of Theorem 2.12 that is independent from Theorem 2.17 .

Definition 2.26 The assessment $\left(\sigma, \beta_{2}\right)$ in the signaling game $\mathcal{S}$ is said to be (fully) consistent if there exists a sequence $\left\{\sigma_{1}^{k}\right\}_{k=1}^{\infty}$ of player 1 strategies such 
that

$$
\begin{aligned}
\sigma_{1}^{k} & \in \prod_{t \in T} \triangle^{0}(S) \text { for all } k \in \mathcal{N} \\
\sigma_{1}(s \mid t) & =\lim _{k \rightarrow \infty} \sigma_{1}^{k}(s \mid t) \text { for all } t \in T \\
\beta_{2}(t \mid s) & =\lim _{k \rightarrow \infty} \frac{p(t) \cdot \sigma_{1}^{k}(s \mid t)}{\sum_{t^{\prime} \in T} p\left(t^{\prime}\right) \cdot \sigma_{1}^{k}\left(s \mid t^{\prime}\right)} \text { for all } t \in T \text { and all } s \in S
\end{aligned}
$$

From the definition one can see that consistency implies Bayesian consistency, but generally for extensive form games, consistency doesn't follow from Bayesian consistency. We show that for signaling games the concepts are equivalent.

Lemma 2.27 Let $\mathcal{S}$ be a signaling game. Then the assessment $\left(\sigma, \beta_{2}\right)$ satisfies Bayesian consistency if and only if it is consistent.

Proof. As we mentioned above the if-part is obvious. For the only-if-part we construct the sequence $\left\{\sigma_{1}^{k}\right\}_{k=1}^{\infty}$ in the following way

$$
\sigma_{1}^{k}(s \mid t):=\frac{\sigma_{1}(s \mid t)+\frac{\beta_{2}(t \mid s)}{k \cdot p(t)}+\frac{1}{k^{2}}}{1+\sum_{s^{\prime} \in S}\left(\frac{\beta_{2}\left(t \mid s^{\prime}\right)}{k \cdot p(t)}+\frac{1}{k^{2}}\right)}
$$

Notice that $\sigma_{1}(s \mid t)=\lim _{k \rightarrow \infty} \sigma_{1}^{k}(s \mid t)$. We write

$$
\frac{p(t) \cdot \sigma_{1}^{k}(s \mid t)}{\sum_{t^{\prime} \in T} p\left(t^{\prime}\right) \cdot \sigma_{1}^{k}\left(s \mid t^{\prime}\right)}=\frac{p(t) \cdot \frac{\sigma_{1}(s \mid t)+\frac{k_{2}(t \mid s)}{k \cdot p(t)}+\frac{1}{k^{2}}}{1+\sum_{s^{\prime} \in S}\left(\frac{\left.k_{2}(t) \cdot s^{\prime}\right)}{k \cdot p^{\prime}(t)}+\frac{1}{k^{2}}\right)}}{\sum_{t^{\prime} \in T} p\left(t^{\prime}\right) \cdot \frac{\sigma_{1}\left(s \mid t^{\prime}\right)+\frac{\beta_{2}\left(t^{\prime} \mid s\right)}{k \cdot p\left(t^{\prime}\right)}+\frac{1}{k^{2}}}{1+\sum_{x^{\prime} \in S}\left(\frac{\left(\beta_{2} \cdot t^{\prime} \mid s^{\prime}\right)}{k \cdot p\left(t^{\prime}\right)}+\frac{1}{k^{2}}\right)}}
$$

and distinguish the following two cases:

(i) Given the signal $s$ there exists a type $t^{\prime}$ such that $\sigma_{1}\left(s \mid t^{\prime}\right) \neq 0$. Then, since for large $k$ the $k$-dependent tends to zero the sequence tends to

$$
\frac{p(t) \cdot \sigma_{1}(s \mid t)}{\sum_{t^{\prime} \in T} p\left(t^{\prime}\right) \cdot \sigma_{1}\left(s \mid t^{\prime}\right)}=\beta_{2}(t \mid s) .
$$

Since the assessment $\left(\sigma, \beta_{2}\right)$ is Bayesian consistent by assumption, we have (full) consistency for this case.

(ii) Given the signal $s$ it holds that $\sigma_{1}(s \mid t)=0$ for all types $t$. Then the mumerator reads

$$
p(l) \cdot \frac{\sigma_{1}(s \mid t)+\frac{\beta_{2}(t \mid s)}{k \cdot p(t)}+\frac{1}{k^{2}}}{1+\sum_{s^{\prime} \in S}\left(\frac{\beta_{2}\left(t \mid s^{\prime}\right)}{k \cdot p(t)}+\frac{1}{k^{2}}\right)}=\frac{1}{k} \frac{\beta_{2}(t \mid s)+\frac{p(t)}{k}}{1+\sum_{s^{\prime} \in S}\left(\frac{\beta_{2}\left(t \mid s^{\prime}\right)}{k \cdot p(t)}+\frac{1}{k^{2}}\right)}
$$


whereas the denomitor reads

$$
\frac{1}{k} \sum_{t^{\prime} \in T} \frac{\beta_{2}\left(t^{\prime} \mid s\right)+\frac{p\left(t^{\prime}\right)}{k}}{1+\sum_{s^{\prime} \in S}\left(\frac{\beta_{2}\left(t^{\prime} \mid s^{\prime}\right)}{k \cdot p\left(t^{\prime}\right)}+\frac{1}{k^{2}}\right)}
$$

yielding

$$
\lim _{k \rightarrow \infty} \frac{p(t) \cdot \sigma_{1}^{k}(s \mid t)}{\sum_{t^{\prime} \in T} p\left(t^{\prime}\right) \cdot \sigma_{1}^{k}\left(s \mid t^{\prime}\right)}=\frac{\beta_{2}(t \mid s)}{\sum_{t^{\prime} \in T} \beta_{2}\left(t^{\prime} \mid s\right)}=\beta_{2}(t \mid s)
$$

Theorem 2.28 Suppose that the assessment $\left(\sigma_{1}, \sigma_{2}, \beta\right)$ is a sequential equilibrium of a signaling game $\mathcal{S}=\left(T, M, A, p, u_{1}, u_{2}\right)$. Then there exists a preference conjecture equilibrium $\left(\mu_{12}, u_{12},\left(\mu_{21}(m), u_{21}(m)\right)_{m \in M^{*}}\right)$ with $u_{21}\left(h_{0}\right)=u_{1}$ and $u_{12}=u_{2}$ that induces $\left(\sigma_{1}, \sigma_{2}, \beta\right)$.

Proof. We start with constructing a conjecture profile $c$. Define $\mu_{21}\left(h_{0}\right):=$ $\sigma_{1}$ and $\mu_{12}:=\sigma_{2}$. Let $m \in M$. For the definition of $\mu_{21}(m)$ we distinguish two cases:

Case 1: there exists an $s_{1}^{\prime} \in S_{1}(m)$ such that $\sigma_{1}\left(s_{1}^{\prime}\right)>0$. Then we define

$$
\mu_{21}(m)\left(s_{1}\right):=\frac{\mu_{21}\left(h_{0}\right)\left(s_{1}\right)}{\sum_{s_{1}^{\prime} \in S_{1}(m)} \mu_{21}\left(h_{0}\right)\left(s_{1}^{\prime}\right)} \text { for all } s_{1} \in S_{1}(m)
$$

Case 2: $\sigma_{1}\left(s_{1}^{\prime}\right)=0$ for all $s_{1}^{\prime} \in S_{1}(m)$. Choose for every $t \in T$ a strategy $s_{1}^{t, m} \in S_{1}(m)$ such that $s_{1}^{t, m}(t)=m$ and $s_{1}^{t, m}\left(t^{\prime}\right) \neq m$ for all $t^{\prime} \neq t$ and define

$$
\widetilde{\mu}_{21}(m)\left(s_{1}^{t, m}\right):=\frac{\beta(t \mid m)}{p(t)}
$$

for all $t \in T$ and

$$
\tilde{\mu}_{21}(m)\left(s_{1}\right)=0
$$

for all $s_{1} \in S_{1}(m)$ with $s_{1} \neq s_{1}^{l, m}$ for all $t \in T$. Next normalize for all $s_{1} \in$ $S_{1}(m)$ :

$$
\mu_{21}(m)\left(s_{1}\right)=\frac{\widetilde{\mu}_{21}(m)\left(s_{1}\right)}{\sum_{s_{1}^{\prime} \in S_{1}(m)} \widetilde{\mu}_{21}(m)\left(s_{1}^{\prime}\right)}
$$

which is well-defined since there exist $s_{1}^{\prime} \in S_{1}(m)$ such that $\tilde{\mu}_{21}(m)\left(s_{1}^{\prime}\right) \neq 0$, namely those strategies $s_{1}^{t, m}$ with $\beta(t \mid m)>0$.

Before completing the construction of $c$ we show that the ingredients defined thus far induce $\left(\sigma_{1}, \sigma_{2}, \beta\right)$. For this we still have to prove (2.3). Let $t \in T$ and 
$m \in M$. If case 1 above applies, then

$$
\begin{aligned}
\beta(t \mid m) & =\frac{p(t) \cdot \sigma_{1}(m \mid t)}{\sum_{t^{\prime} \in T} p\left(t^{\prime}\right) \cdot \sigma_{1}\left(m \mid t^{\prime}\right)} \\
& =\frac{p(t) \cdot \sum_{s_{1}: s_{1}(t)=m} \sigma_{1}\left(s_{1}\right)}{\sum_{t^{\prime} \in T} p\left(t^{\prime}\right) \cdot \sum_{s_{1}: s_{1}\left(t^{\prime}\right)=m} \sigma_{1}\left(s_{1}\right)} \\
& =\frac{p(t) \cdot \sum_{s_{1}: s_{1}(t)=m} \mu_{21}\left(h_{0}\right)\left(s_{1}\right)}{\sum_{t^{\prime} \in T} p\left(t^{\prime}\right) \cdot \sum_{s_{1}: s_{1}\left(t^{\prime}\right)=m} \mu_{21}\left(h_{0}\right)\left(s_{1}\right)} \\
& =\frac{\sum_{s_{1}: s_{1}(t)=m} p(t) \cdot \mu_{21}(m)\left(s_{1}\right)}{\sum_{t^{\prime} \in T} \sum_{s_{1}: s_{1}\left(t^{\prime}\right)=m} p\left(t^{\prime}\right) \mu_{21}(m)\left(s_{1}\right)},
\end{aligned}
$$

so (2.3) holds. If case 2 above applies, then

$$
\begin{aligned}
\frac{\sum_{s_{1}: s_{1}(t)=m} p(t) \cdot \mu_{21}(m)\left(s_{1}\right)}{\sum_{t^{\prime} \in T} \sum_{s_{1}: s_{1}\left(t^{\prime}\right)=m} p\left(t^{\prime}\right) \cdot \mu_{21}(m)\left(s_{1}\right)} & =\frac{\sum_{s_{1}: s_{1}(t)=m} p(t) \cdot \tilde{\mu}_{21}(m)\left(s_{1}\right)}{\sum_{t^{\prime} \in T} \sum_{s_{1}: s_{1}\left(l^{\prime}\right)=m} p\left(t^{\prime}\right) \cdot \tilde{\mu}_{21}(m)\left(s_{1}\right)} \\
& =\frac{\beta(t \mid m)}{\sum_{t^{\prime} \in T} \beta\left(t^{\prime} \mid m\right)} \\
& =\beta(t \mid m),
\end{aligned}
$$

so also in this case (2.3) holds. Hence, $c$ induces $\left(\sigma_{1}, \sigma_{2}, \beta\right)$.

We now complete the construction of $c$. Let $u_{12}:=u_{2}, u_{21}\left(h_{0}\right):=u_{1}$, and for all $t \in T, m, m^{\prime} \in M$, and $a \in A$ let

$$
u_{21}(m)\left(t, m^{\prime}, a\right):=0
$$

In order to show that $c$ is a preference conjecture equilibrium first note that it satisfies Bayesian updating: for $m \in M$ such that $\sum_{s_{1}^{\prime} \in S_{1}(m)} \mu_{21}\left(h_{0}\right)\left(s_{1}^{\prime}\right)>0$, case 1 above applies, so Bayesian updating follows immediately.

Finally, we show optimality of $c$. Let $s_{1} \in S_{1}$. If $\mu_{21}\left(h_{0}\right)\left(s_{1}\right)>0$ then $\sigma_{1}\left(s_{1}\right)>$ 0 , so optimality of $\sigma_{1}$ with respect to $\sigma_{2}$ yields that $s_{1}$ is optimal with respect to $\sigma_{2}$ in $\mathcal{S}$. Hence $s_{1}$ is optimal with respect to $\mu_{12}$ and $u_{21}\left(h_{0}\right)$. Further, for every $m \in M, s_{1}$ is optimal with respect to $\mu_{12}$ and $u_{21}(m)$ since player 1 is indifferent between all messages $m$ by definition of $u_{21}(m)$. This shows optimality on the part of player 1 .

Let $s_{2} \in S_{2}$ such that $\mu_{12}\left(s_{2}\right)>0$. Then $\sigma_{2}\left(s_{2}\right)>0$ and optimality of $\sigma_{2}$ with respect to $\beta$ implies that $s_{2}$ is optimal with respect to $\beta$ on any $m \in M$, that is

$$
U_{2}\left(m, s_{2}, \beta\right) \geq U_{2}\left(m, s_{2}^{\prime}, \beta\right)
$$

for all $s_{2}^{\prime} \in S_{2}$ and all $m \in M$. Since

$$
U_{2}\left(m, s_{2}, \beta\right)=\sum_{t \in T} \beta(t \mid m) \cdot u_{2}\left(t, m, s_{2}(m)\right)
$$


(2.8) implies, together with (2.3) and $u_{12}=u_{2}$

$$
\begin{aligned}
U_{12}\left(m, s_{2}, u_{12}, \mu_{21}(m)\right) & =\sum_{t \in T} p(t) \sum_{s_{1}: s_{1}(t)=m} \mu_{21}(m)\left(s_{1}\right) \cdot u_{12}\left(t, m, s_{2}(m)\right) \\
& \geq \sum_{t \in T} p(t) \sum_{s_{1}: s_{1}(t)=m} \mu_{21}(m)\left(s_{1}\right) \cdot u_{12}\left(t, m, s_{2}^{\prime}(m)\right) \\
& =U_{12}\left(m, s_{2}^{\prime}, u_{12}, \mu_{21}(m)\right)
\end{aligned}
$$

for all $s_{2}^{\prime} \in S_{2}$ and $m \in M$. Hence, $s_{2}$ is optimal for player 2 with respect to $\mu_{21}(m)$ and $u_{12}$. This completes the proof. 


\section{Chapter 3}

\section{An epistemic approach to uncertainty about the opponents' utilities}

\subsection{Introduction}

In noncooperative games in which the players' utility functions are commonly known among the players [Tan \& Werlang, 1988] links the iterative elimination of strictly dominated strategies to the concept of common belief in rationality. Here, with iterative elimination of strictly dominated strategies we mean the process in which players iteratively eliminate pure strategies for which their utility is always lower than for some other (possibly mixed) strategy choice and consider reduced games that arise from assuming that their opponents will follow the same elimination process. With common belief in rationality we mean the principle that every player is rational (that is, behaves rationally given his beliefs), believes that his opponents are rational, believes that his opponents believe that the other players are rational and so on. The result from [Tan \& Wcrlang, 1988] says that a strategy survives the iterative elimination of strictly dominated strategies if and only if it can rationally be chosen under common belief in rationality.

The purpose of this chapter is to generalize this result to the situation in which the players are uncertain about their opponents' utility functions. We assume that every player has uncertainty about opponent i's utility function, but believes this function to be in some class $U_{i}$ that is commonly known among all players. This implies that every player may have a different belief about some opponent $i$ 's utility function, but all these beliefs are in the set of all probability distributions over $U_{i}$. Furthermore, we assume that every player knows his own utility function.

The framework we use to analyze the described situation is that of players' 
types in accordance with Harsanyi's model of games with incomplete information. A type contains information about the player's belief about opponents' strategy choices, utility functions and beliefs. Harsanyi aggregation is a concept in group clecision making theory that assigns a group preference relation to a set of individual preference relations. For player $i$ we consider preference relations induced by different utility functions in $U_{i}$ and different beliefs over the opponents" strategy choice and define "closedness under Harsanyi aggregation". The set of induced preference relations is closed under Harsanyi aggregation of for every pair $\succeq_{1}, \succeq_{2}$ of preference relations there exists another preference relation $\succeq_{3}$ such that $\succeq_{3}$ is equivalent to the Harsanyi aggregation of $\succeq_{1}$ and $\succeq_{2}$. Our main result states that if the set of preference relations is closed under Harsanyi aggregation a strategy can rationally be chosen under common belief in rationality if and only if it survives the iterative elimination of strictly dominated strategies. The concept of Harsanyi aggregation has not been applied in this contcxt in literature. An approach to elimination of dominated strategies in the context of rationalizability in games with incomplete information can be found in [Battigalli, 2003].

The outline of this chapter is as follows. In Section 3.2 we summarize the epistemic framework needed to state the result from [Tan \& Wcrlang, 1988] for the case in which there is no uncertainty among players about utility functions. In Section 3.3 we formalize the epistemic model to analyze situations in which players do have uncertainty about their opponents' utility functions. Next we define closedness under Harsanyi aggregation and show that this is a sufficient condition for an equivalence result analogous to [Tan \& Werlang, 1988]. At the end of the chapter we analyze a textbook examplc, Cournot duopoly, for the case in which two firms have uncertainty about each other's marginal cost function and apply our main theorem to calculate the set of strategies that can rationally be chosen under common belief in rationality for both firms.

\subsection{Rationality with commonly known utility func- tions}

In this section we present the epistemic foundation that leads to the result from [Tan \& Werlang, 1988] for the case in which we consider all utility functions to be "commonly known". By commonly known we mean that player $i$ knows the utility function of player $j$ (for all $j$ ), but also that every playcr $j$ knows that $i$ knows his utility functions and so on. Loosely speaking we consider the case in which we consider all utility functions to be given and present the relationship between strategies that can be chosen under "common belief in rationality" and strategies that survive the "iterative elimination of strictly dominated strategies". 


\subsubsection{The epistemic model}

Let $I$ be a finite set of players. Now, consider the game $\Gamma=\left(I,\left(S_{i}\right)_{i \in I},\left(u_{i}\right)_{i \in I}\right)$, where for every $i \in I$ there is a finite set $S_{i}$ of pure strategies and a utility function

$$
u_{i}: \times_{j \in I} S_{j} \rightarrow \mathbb{R}
$$

In addition to the game, consider an epistemic model $M=\left(\left(T_{i}\right)_{i \in I},\left(b_{i}\right)_{i \in I}\right)$, where for every $i \in I$ there is a set $T_{i}$ of types and a belief $b_{i}$ about the opponents' strategy choices and types

$$
b_{i}: T_{i} \rightarrow \Delta\left(S_{-i} \times T_{-i}\right)
$$

where $S_{-i}:=\times_{j \neq i} S_{j}$ and $T_{-i}:=\times_{j \neq i} T_{j}$. Notice that for every $i$ the fucntion $b_{i}$ recursively refers the own type. Namely, player $i$ holds a belief about the strategy choices and types of his opponents. But every opponent $j \neq i$ holds a belief in $\Delta\left(S_{-j} \times T_{-j}\right)$. Thereforc, player $i$ has uncertainty about elements in

$$
\Delta\left(S_{-i} \times\left(\times_{j \neq i} \Delta\left(S_{-j} \times T_{-j}\right)\right)\right) .
$$

This process can be repeated and we obtain a sequence that we call a belief hierarchy.

For every pair $\left(s_{i}, t_{i}\right) \in S_{i} \times T_{i}$ we introduce the "expected utility"

$$
u\left(s_{i}, t_{i}\right):=\sum_{s_{-i} \in S_{-i}} b_{i}\left(t_{i}\right)\left(s_{-i}\right) u_{i}\left(s_{i}, s_{-i}\right)
$$

Here,

$$
b_{i}\left(t_{i}\right)\left(s_{-i}\right)
$$

denotes the probability that type $t_{i}$ attaches to $s_{-i}\left(b_{i}\left(t_{i}\right)\left(s_{-i}\right)\right.$ is the marginal probability with respect to $\left.S_{-i}\right)$. Thus the expected utility $u\left(s_{i}, t_{i}\right)$ denotes the utility that player $i$ expects when playing the strategy $s_{i}$ and holding the belief $b_{i}\left(t_{i}\right)$. The pair $\left(s_{i}, t_{i}\right)$ is called "rational" if

$$
u\left(s_{i}, t_{i}\right) \geq u\left(s_{i}^{\prime}, t_{i}\right) \text { for all } s_{i}^{\prime} \in S_{i}
$$

We say that type $t_{i}$ believes an event

$$
E \subseteq S_{-i} \times T_{-i}
$$

if

$$
b_{i}\left(t_{i}\right)(E)=1
$$

\subsubsection{Common belief in rationality}

In order to formalize the consequences of the hierachy that is induced by the definition of $b_{i}$ for all $i \in I$ we iteratively define the sets 


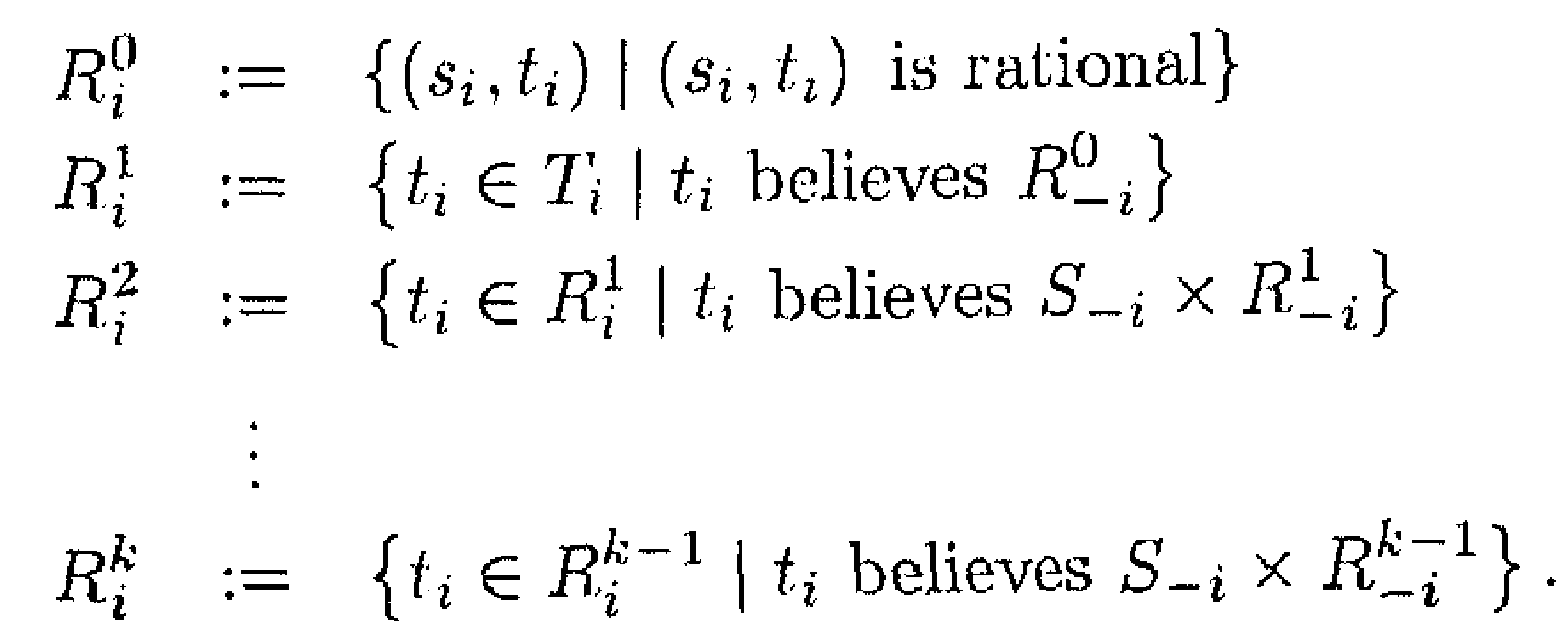

Here,

$$
R_{-i}^{k}:=\times_{j \neq i} R_{j}^{k}
$$

Furthermore, let

$$
R_{i}^{\infty}:=\bigcap_{k \in \mathbb{N}} R_{i}^{k}
$$

Hence, the set $R_{i}^{0}$ is the set of all rational pairs $\left(s_{i}, t_{i}\right)$ for player $i$. The set $R_{i}^{1}$ contains those types $t_{i}$ that believe that every opponent $j$ will only choose rational pairs $\left(s_{j}, t_{j}\right)$. Hence, the set $R_{i}^{1}$ formalizes the fact that player $i$ believes that every opponent $j \neq i$ is rational. The set $R_{i}^{2}$ contains all types $t_{i}$ that believe the strategy profile of the opponents to be $S_{-i}$ and believe that every opponent $j$ is of a type in $R_{j}^{1}$. Thus, $R_{i}^{2}$ reflects the fact that player $i$ believes that every opponent $j \neq i$ believes that player $i$ is rational. Notice that $R_{i}^{1} \supseteq$ $R_{i}^{2} \supseteq R_{i}^{3} \supseteq \ldots$ The types in $R_{i}^{\infty}$ are the types that "survive" the iterative process we just described, or, more exactly, the types $t_{t}$ that believe that their opponents are rational, believe that their opponents believe that all the players are rational, believe that all the opponents believe that every player believes his opponents to be rational and so on. The fact that every player $i$ only considers types in $R_{i}^{\infty}$ is called "common belief in rationality". We say that a strategy $s_{i}$ can "rationally be chosen under common belief in rationality" if there exist a $t_{i} \in R_{i}^{\infty}$ such that $\left(s_{i}, t_{i}\right)$ is rational.

\subsubsection{Dominated strategies}

Let

$$
D_{i} \subseteq S_{i} \text { and } D_{-i} \subseteq S_{-i}
$$

Then $s_{i}$ is "strictly dominated" on $D_{i} \times D_{-i}$ if there exists a $\mu_{i} \in \Delta\left(D_{i}\right)$ such that

$$
u_{i}\left(\mu_{i}, s_{-i}\right)>u_{i}\left(s_{i}, s_{-i}\right) \forall s_{-i} \in D_{-i} .
$$


We now define "iterative elimination of strictly dominated strategies" by

$$
\begin{aligned}
D_{i}^{0}: & =S_{i} \\
D_{i}^{1}: & =\left\{s_{i} \in D_{i}^{0} \mid s_{i}\right. \text { is not } \\
& \text { strictly dominated on } \left.D_{i}^{0} \times D_{-i}^{0}\right\} \\
& \vdots \\
D_{i}^{k}: & =\left\{s_{i} \in D_{i}^{k-1} \mid s_{i}\right. \text { is not } \\
& \text { strictly dominated on } \left.D_{i}^{k-1} \times D_{-i}^{k-1}\right\}
\end{aligned}
$$

Here,

$$
D_{-i}^{k}:=\times_{j \neq i} D_{j}^{k}
$$

Furthermore, let

$$
D_{i}^{\infty}:=\bigcap_{k \in \mathbb{N}} D_{i}^{k}
$$

The structure of the iterative definitions is the same as in the last paragraph. In the iterative process every player $i$ first eliminates his strategies that are strictly dominated (the set $D_{i}^{1}$ ). In the next step player $i$ first assumes that every opponent $j \neq i$ follows the same elimination procedure and then eliminates strategies that are dominated in the "reduced game" that for every player only contains strategies that survived the first elimination step. This procedure is then iterated. A strategy $s_{i}$ is said to "survive iterative climination of strictly dominated strategies" if $s_{i} \in D_{i}^{\infty}$.

Theorem 3.1 (Tan \& Werlang, 1988) The strategy si can rationally be chosen under common belief in rationality if and only if it survives the iterative elimination of strictly dominated strategies.

\subsubsection{Example}

Consider the following game

$\begin{array}{cccc} & c & d & e \\ a & 2,3 & 3,0 & 0,1 \\ b & 0,0 & 1,6 & 4,2 .\end{array}$

Here, player 1 chooses between $a, b$ and player 2 between $c, d, e$. With this example we want to illustrate the relationship between iterative elimination of strictly dominated strategies and common belief in rationality.

First notice that for player 1 there is no strictly dominated strategy, whereas player 2's pure strategy $e$ is strictly dominated by $\frac{1}{2} c+\frac{1}{2} d$. However, after eliminating $e$, player 1's strategy $b$ is strictly dominated by $a$. After iteratively eliminating $e$ and $b$, strategy $d$ is strictly dominated by $c$. Hence, iterative elimination of strictly dominated strategies leads to the unique strategy pair $(a, c)$. 
If we analyze the same game from the viewpoint of common belicf in rationality, we see that rationality of player 1 puts no restrictions on his strategy choice, whereas rationality of player 2 leads him to never choose $e$. If now player 1 is rational and belicves player 2 to be rational, player 1 believes that player 2 will not choose $e$ and therefore, player 1 will not choose $b$. If, on his turn, player 2 is rational and believes that player 1 believes that player 2 is rational, player 2 believes that player 1 will not play $b$ and therefore, player 2 will not choose $d$. Hence, the same strategy pair $(a, c)$ results.

\subsection{Modelling uncertainty about the opponents' utility functions}

\subsubsection{The epistemic model}

Let again $I$ be the finite set of players. For every $i \in I$ let $T_{i}$ denote the set of types of player $i$ and let $U_{i}$ be the set of possible utility functions of player $i$. The interpretation is that all players $i \neq j$ are uncertain about player $j$ 's utility function, but believe this function to be in $U_{j}$. Furthermore let $U:=\times_{i \in I} U_{i}$ and let $S_{i}$ be the (finite) set of pure strategies of player $i$. An epistemic model for $U$ is a tuple

$$
M=\left(\left(T_{i}\right)_{i \in I},\left(U_{i}\right)_{i \in I},\left(b_{i}\right)_{i \in I},\left(u_{i}\right)_{i \in I}\right)
$$

where

$$
\begin{aligned}
b_{i} \quad: \quad T_{i} \rightarrow \Delta\left(S_{-i} \times T_{-i}\right) \\
u_{i}: \quad T_{i} \rightarrow U_{i} .
\end{aligned}
$$

Both $b_{i}$ and $u_{i}$ are onto. Furthermore, $S_{-i}:=\times_{j \neq i} S_{j}$. Hence, the belief about the opponents' utility functions is modelled implicitly by the belief about the opponents' types: different types of player $i$ may have a different utility function (by the function $u_{i}$ ) and hence, by holding a belief about player $i$ 's type, players hold a belif about player $i$ 's utility function.

A type $t_{i}$ has a utility function $u_{i}\left(t_{i}\right)$ and a belicf $b_{i}\left(t_{i}\right)$. Given this utility function and this belief, wo introduce the notation

$$
u_{i}\left(s_{i}, t_{i}\right):=\sum_{s_{-i} \in S_{-i}} b_{i}\left(t_{i}\right)\left(s_{-i}\right) u_{i}\left(t_{i}\right)\left(s_{i}, s_{-i}\right)
$$
for player $i$ 's expected utility w.r.t. $u_{i}\left(t_{i}\right)$ and $b_{i}\left(t_{i}\right)$. A pair $\left(s_{i}, l_{i}\right)$ is called
rational if

$$
u_{i}\left(s_{i}, t_{i}\right) \geq u_{i}\left(s_{i}^{\prime}, t_{i}\right) \text { for all } s_{i}^{\prime} \in S_{i}
$$

We say that player $i$ with type $t_{i}$ believes an event $E \subseteq\left(S_{-i} \times T_{-i}\right)$ if

$$
b_{i}\left(t_{i}\right)(E)=1
$$




\subsubsection{Common belief in rationality}

In analogy to section 3.2 .2 we model the iterative belief hierarchy for all $i$ by defining the sets

$$
\begin{array}{lll}
R_{i}^{0}(U):= & \left\{\left(s_{i}, t_{i}\right) \mid\left(s_{i}, t_{i}\right) \text { is rational }\right\} \\
R_{i}^{1}(U):=\quad & \left\{t_{i} \in T_{i} \mid t_{i} \text { believes } R_{-i}^{0}\right\} \\
R_{i}^{2}(U):= & \left\{t_{i} \in R_{i}^{1} \mid t_{i} \text { believes } S_{-i} \times R_{-i}^{1}\right\} \\
& \vdots & \\
R_{i}^{k}(U):= & \left\{t_{i} \in R_{i}^{k-1} \mid t_{i} \text { believes } S_{-i} \times R_{-i}^{k-1}\right\} .
\end{array}
$$

Here,

$$
R_{-i}^{k}(U):=\times_{j \neq i} R_{j}^{k}(U)
$$

We write the set $U$ in the argument to inclicate that the sets $R_{i}^{k}(U)$ depend on $U$ since the set of types depends on $U$ (player $i$ 's utility function is modelled by his type and the function $u_{i}$ that attaches a utility function to every type $t_{i}$ ). Furthermore, let

$$
R_{i}^{\infty}(U):=\bigcap_{k \in \mathbb{N}} R_{i}^{k}(U)
$$

A strategy $s_{i}$ is called rationalizable w.r.t. $U$ if there exist an epistemic model $M$ for $U$ and a $t_{i} \in R_{i}^{\infty}$ such that $\left(s_{i}, t_{i}\right)$ is rational. The interpretation of the iterative procedure is as clexcribed in section 3.2.2.

\subsubsection{Dominated strategies}

Next we formalize the iterative elimination of strictly dominated strategies for the case in which players are uncertain about the opponents' utility functions. For this purpose we first define what it means in this context that a strategy is strictly dominated. In accordance with section 3.2 .3 let

$$
D_{i} \subseteq S_{i} \text { and } D_{-i} \subseteq S_{-i}
$$

A strategy $s_{i}$ is strictly dominated on $D_{i}^{k-1} \times D_{-i}^{k-1}$ w.r.t. $U_{i}$ if there exists a $\mu_{i} \in \Delta\left(D_{i}^{k-1}\right)$ such that

$$
u_{i}\left(\mu_{i}, s_{-i}\right)>u_{i}\left(s_{i}, s_{-i}\right) \forall s_{-i} \in D_{-i}^{k-1} \forall u_{i} \in U_{i} .
$$

Hence, the difference with section 3.2 .3 is that we demand the strict inequality for all $u_{i} \in U_{i}$. The intuition is analogous to section 3.2.3: a strategy $s_{i}$ is strictly dominated if it is never optimal for player $i$, no matter what player $i$ believes his opponents to choose and no matter with respect to which utility function in $U_{i}$ player $i$ evaluates the possible outcomes of the game. Now, for all $i$ we iteratively define the sets

$$
\begin{array}{ll}
D_{i}^{0}(U):= & S_{i} \\
D_{i}^{k}(U):=\quad\left\{s_{i} \in \begin{array}{l}
D_{i}^{k-1} \mid s_{i} \text { is not strictly dominated on } D_{i}^{k-1} \times D_{-i}^{k-1} \\
\end{array}\right. & \text { w.r.t. } \left.U_{i}\right\}
\end{array}
$$


Here,

$$
D_{-i}^{k}:=\times_{j \neq i} D_{j}^{k}
$$

A strategy $s_{i}$ is said to survive iterated elimination of strictly dominated strategies w.r.t. $U$ if $s_{i} \in D_{i}^{\infty}(U)$ where

$$
D_{i}^{\infty}(U):=\bigcap_{k \in \mathbb{N}} D_{i}^{k}(U)
$$

By writing $U$ in the argument we indicate that the elimination procedure depends on $U$, since it is part of the notion of strategies that are strictly dominated

\subsubsection{An example}

The main aim of this chapter is to answer the question whether a generalization of the Tan-Werlang result exists for the case in which players are uncertain about the opponents' utility functions. The first idea to generalize this result is the following attempt.

Conjecture 3.2 The strategy $s_{i}$ can rationally be chosen under common belief in rationality (w.r.t. $U$ ) if and only if it survives the iterative elimination of strictly dominated strategies (w.r.t. U).

The next example shows that this conjecture cannot hold in general.

Example 3.3 Consider a game in which player 1 chooses between $a, b, c$ and player 2 chooses between $d, e$. The utility functions $u_{1}$ and $u_{1}^{\prime}$ are given by

$\begin{array}{ccccccc}\mathbf{u}_{1} & d & e & & \mathbf{u}_{1}^{\prime} & d & e \\ a & 2 & 0 & \text { and } & a & 4 & -1 \\ b & -1 & 4 & & b & 0 & 2 \\ c & 1 & 1 & & c & 1 & 1\end{array}$

We assume that player 2 believes that his opponent's utility function is in the closed and convex set

$$
U_{1}:=\left\{\lambda u_{1}+(1-\lambda) u_{1}^{\prime} \mid \lambda \in[0,1]\right\} .
$$

Hence, player 2 believes his opponent's expected utility to be of the form

$$
\begin{array}{ccc}
\lambda \mathbf{u}_{1}+(1-\lambda) \mathbf{u}_{1}^{\prime} & d & e \\
a & 4-2 \lambda & \lambda-1 \\
b & -\lambda & 2+2 \lambda \\
c & 1 & 1
\end{array}
$$

Assume that player 1 believes with probability $\alpha$ that player 2 chooses $d$ and believes with probability $(1-\alpha)$ that player 2 chooses $e$. Then $c$ is optimal if

$$
\begin{aligned}
\alpha(4-2 \lambda)+(1-\alpha)(\lambda-1) & \leq 1 \\
\alpha(-\lambda)+(1-\alpha)(2+2 \lambda) & \leq 1 .
\end{aligned}
$$


This is equivalent to

$$
\alpha \leq \frac{2-\lambda}{5-3 \lambda}
$$

and

$$
\alpha \geq \frac{1+2 \lambda}{3 \lambda+2}
$$

respectively. Hence, a necessary condition is

$$
\frac{1+2 \lambda}{3 \lambda+2} \leq \frac{2-\lambda}{5-3 \lambda},
$$

which, however, has no solution for $\lambda \in[0,1]$. Therefore, $c$ is never a rational choice for any $\bar{u}_{1} \in U_{1}$. On the other hand, there is no mixed strategy $\mu_{i}$ such that $c$ is strictly dominated by $\mu_{i}$ for every $\bar{u}_{1} \in U$. Namely, if $c$ is strictly dominated by $\beta a+(1-\beta) b$ w.r.t. $u_{1}$ it holds that

$$
\frac{2}{3}<\beta<\frac{3}{4}
$$

If $c$ is strictly dominated by $\beta a+(1-\beta) b$ w.r.t. $u^{\prime}$ then

$$
\frac{1}{4}<\beta<\frac{1}{3} .
$$

Hence, there is no $\beta$ such that $c$ is dominated by $\beta a+(1-\beta) b$ for every $u \in U$. Thus, we showed that player 1's strategy $c$ is never a best response, but not strictly dominated (w.r.t. $U$ ) by some a mixed strategy.

\subsection{Equivalence between common belief in ra- tionality and iterative elimination}

\subsubsection{Closedness under Harsanyi aggregation}

Let $Z$ be the set of pure outcomes of the game and let $\succeq_{i}$ be a preference relation on $\Delta(Z)$. A function

$$
v: \Delta(Z) \rightarrow \mathbb{R}
$$

is called a representing utility function for $\succeq_{i}$ if

$$
v(a) \geq v(b) \Leftrightarrow a \succeq_{i} b .
$$

Let $a$ be a probability distribution on the pure outcomes $z_{k} \in Z$,

$$
a=\sum_{i=1}^{|Z|} a_{k} z_{k}
$$


with $\sum_{k^{\prime}} a_{k}=1$. Then the utility function $v$ is linear if for every probability distribution $a \in \Delta(Z)$ it holds that

$$
v(a)=\sum_{k=1}^{|Z|} a_{k} v\left(z_{k}\right) .
$$

The following axioms and representation theorem are due to Marshak.

Axiom 3.4 The relation $\succeq_{i}$ is a complete, transitive relation on $\Delta(Z)$.

Axiom 3.5 It holds that

$$
a \succeq_{i} b \succeq_{i} c \Rightarrow \exists \lambda \in[0,1] \text { s.t. } \lambda a+(1-\lambda) c \approx_{i} b .
$$

Axiom $3.6 a \succeq b \succeq_{i} c \succeq_{i} d$ for some $a, b, c, d \in \Delta(Z)$.

Axiom 3.7 For all $a \in \Delta(Z)$ and all $\lambda \in[0,1]$ il holds that

$$
b \approx_{i} c \Rightarrow \lambda a+(1-\lambda) b \approx_{i} \lambda a+(1-\lambda) c .
$$

Theorem 3.8 Given Axioms $1-4$ there exists a representing linear utility function $\%$. This is unique up to a positive affine transformation.

Hence, the Marshak axioms desrcibe a class of preference relations for an individual decision maker. Now consider the following context. A group of individual decision makers is asked for their preference relation as a group. In this context of "group decision making" the group must agree upon some procedure to generate the group's preference relation $\succeq_{0}$ from the individual preference relations $\succeq_{i}$. One way to do this is by the so called "Harsanyi aggregation". Let $I$ be a set of individuals $i$ each holding a preference relation $\succeq_{i}$. Furthermore, let $i, j \in I$ and let $P, P_{i}, Q \in \Delta(Z)$.

Axiom 3.9 Every $\succeq_{i}$ satisfies Marshak's axioms.

Axiom 3.10 The group's preference relation $\succeq_{0}$ satisfies Marshak's axioms.

Axiom 3.11 (functionality) $P \approx_{0} Q$ if $P \approx_{i} Q$ for all $i \in I$.

Axiom 3.12 (uniqueness) $\exists Q \forall i \exists P \forall j \neq i$ it holds that $P \succ_{i} Q$ but $P \approx_{j} Q$.

Axiom 3.13 (positivity) $P \succ_{0} Q$ if $P \succeq_{i} Q$ for all $i$ and $P \succ_{j} Q$ for some $j$.

Theorem 3.14 (Harsanyi's Aggregation Theorem) The Harsanyi axioms (axioms 3.9 to 3.13) imply the existence of expected utility functions $v_{0}, v_{i}$, for every $i \in I$, for the preference relations $\succeq_{i}$ and $\succeq_{0}$ such that $v_{0}=\sum_{i \in I} v_{i}$.

Next we turn to the epistemic model with sets of utility functions. In our context, every player doesn't know the preference relation of his opponents but believes these relations to be in certain classes that we will denote by $\Lambda_{i}$. For $\succeq_{i}^{1}, \succeq_{i}^{2} \in \Lambda_{i}$ we say that $\succeq_{0}$ is a Harsanyi aggregation if $\left\{\succeq_{i}^{1}, \succeq_{i}^{2}, \succeq_{0}\right\}$ satisfies the Harsinyi axioms. 
Definition 3.15 Let $\Lambda_{i}$ be a set of preference relations on $\Delta(Z)$ satisfying the Marshak axioms. Then $\Lambda_{i}$ is called closed under Harsanyi aggregation if for every $\succeq_{i}^{1}, \succeq_{i}^{2} \in \Lambda_{i}$ and every Harsanyi aggregation $\succeq_{0}$ of $\left(\succeq_{i}^{1}, \succeq_{i}^{2}\right)$ it holds that $\succeq_{0} \in \Lambda_{i}$.

Next we investigate the relationship between the set of preference relations and the existence of a set of utility functions. All preforence relations $\succeq_{i}$ satisfy Marshak's axioms. Hence, for every $\succeq_{i}$ there exists a $v_{i}\left(\succeq_{i}\right)$ such that $v_{i}\left(\succeq_{i}\right)$ is a linear representing utility function for $\succeq_{i}$. As we noted before, $v_{i}\left(\succeq_{i}\right)$ is determined uniquely up to an affine transformation. Closedness under Harsanyi aggregation in terms of utility functions means that the utility function $v_{0}$, representing the aggregation of two preference relations, is in the class $V_{i}\left(\Lambda_{i}\right)$ of representing utility functions. These classes are formally defined by

$$
V_{i}\left(\Lambda_{i}\right):=\left\{v_{i} \mid v_{i} \text { represents } \succeq_{i} \text { for some } \succeq_{i} \in \Lambda_{i}\right\} \text {. }
$$

By Harsanyi's theorem it holds that $\Lambda_{i}$ is closed under Harsanyi aggregation if and only if $V_{i}\left(\Lambda_{i}\right)$ is closed under addition. Hence, if a player believes player $i$ 's prefercnce relation (over mixed outcomes) to be in a class $\Lambda_{i}$ that is closed under Harsanyi aggregation, this is equivalent to believing that player $\imath$ 's utility function is in a class $V_{i}\left(\Lambda_{i}\right)$ that has the following properties.

1. For all $\succeq_{i} \in \Lambda_{i}$ there exists a $v_{i}\left(\succeq_{i}\right) \in V_{i}\left(\Lambda_{i}\right)$ that represents $\succeq_{i}$.

2. For all $v_{i} \in V_{i}\left(\Lambda_{i}\right)$ and all $c \in \mathbb{R}_{+}, d \in \mathbb{R}$ it holds that $c \cdot v_{i}+d \in V_{i}\left(\Lambda_{i}\right)$.

3. For all $v_{i}^{1}, v_{i}^{2} \in V_{i}\left(\Lambda_{i}\right)$ it holds that $v_{i}^{1}+v_{i}^{2} \in V_{i}\left(\Lambda_{i}\right)$.

If the situation is stated in terms of utility functions rather than in terms of preference relations we call a set $V_{i}\left(\Lambda_{i}\right)$ satisfying the properties 2 and 3 closed under Harsanyi aggregation.

Notice that in terms of our model every $v_{i} \in V_{i}\left(\Lambda_{i}\right)$ corresponds to a certain combination $\left(u_{i}, \mu_{-i}\right) \in U_{i} \times \Delta\left(S_{-i}\right)$. A given $\mu_{-i} \in \Delta\left(S_{-i}\right)$ induces for every $s_{i} \in S_{i}$ a probability distribution over outcomes that can be reached by playing $s_{i}$. If, moreover, the utility function $u_{i}$ is given (and we assume that every player $i$ nows his own utility function) player $i$ can, together with the probability distribution over outcomes we just described, calculate the expected utility for every $s_{i}$. Therefore, we say that every element of the set

$$
P_{i}:=\left\{\left(u_{i}, \mu_{-i}\right) \mid\left(u_{i}, \mu_{-i}\right) \in U_{i} \times \Delta\left(S_{-i}\right)\right\}
$$

"induces" a preference relation $\succeq_{i}$ for player $i$ on $\Delta\left(S_{i}\right)$. Then

$$
\Lambda_{i}:=\left\{\succeq_{i} \mid \succeq_{i} \text { is induced by some }\left(u_{i}, \mu_{-i}\right) \in P_{i}\right\}
$$

Every preference relation in $\succeq_{i} \in \Lambda_{i}$, on its turn, corresponds to a $v_{i} \in V_{i}\left(\Lambda_{i}\right)$ $\left(\succeq_{i}\right.$ satisfies the Marshak axioms). Let $v_{i}\left(u_{i}, \mu_{-i}\right)$ be the vector $\left(u_{i}\left(s_{i}, \mu_{-i}\right)\right)_{s_{i} \in S_{i}}$ and define

$$
\bar{V}_{i}\left(U_{i}, \Delta\left(S_{-i}\right)\right)=\left\{v_{i}\left(u_{i}, \mu_{-i}\right) \mid u_{i} \in U_{i}, \mu_{i} \in \Delta\left(S_{-i}\right)\right\} .
$$


Since the ste $V_{2}\left(\Lambda_{i}\right)$ we defined above includes all utility functions that represent some $\iota_{i} \in \Lambda_{i}$ and we argued that $v_{i} \in V_{i}\left(\Lambda_{i}\right)$ implies that every positive, affine transformation of $l_{i}$ is in $V_{i}\left(\Lambda_{i}\right)$ we define

$$
\begin{aligned}
V_{i}\left(U_{1}, \Delta\left(S_{-i}\right)\right): & =\left\{c \cdot v_{i}\left(u_{i}, \mu_{-i}\right)+d \mid v_{i}\left(u_{i}, \mu_{-i}\right) \in \bar{V}_{i}\left(U_{i}, \Delta\left(S_{-i}\right)\right)\right. \\
& \left.: \quad \in \mathbb{R}_{+}, d \in \mathbb{R}\right\}
\end{aligned}
$$

\subsubsection{Main result}

For the prof of our main result we use the following result (see, for example, [Rockafellatr. 1970]).

Theorem 3.16 (Separating Hyperplane Theorem) Let $U, V \subset \mathbb{R}^{n}$ be two conver sfts and let $U \cap V=\varnothing$. Furthermore, let $U$ and $V$ be closed. Then there ixists a rector $r \in \mathbb{R}^{n}$ such that

$$
\begin{aligned}
& x \cdot u>0 \text { for all } u \in U \\
& x \cdot v<0 \text { for all } v \in V .
\end{aligned}
$$

The verfor $x$ is the normal of the so called separating hyperplane, the hyperplane that separates the sets $U$ and $V$.

Now we are able to prove the next Theorem.

Theorem 3.17 Let $U_{i}$ be closed for all $i \in I$ and let $V_{i}\left(U_{i}, \Delta\left(S_{-i}\right)\right)$ be closed under Harsanyi aggregation for all $i \in I$. Furthermore, let $D_{j} \subseteq S_{j}$ for all $j$.
Then $s_{i} \in D_{i}$ is not strictly dominated Then $s_{i} \in D_{i}$ is not strictly dominated w.r.t. $U$ on $D_{i} \times D_{-i}$ if and only if there exist a $p_{i} \in \Delta\left(D_{-i}\right)$ and $a u_{i} \in U_{i}$ such that

$$
u_{i}\left(s_{i}, p_{i}\right) \geq u_{i}\left(s_{i}^{\prime}, p_{i}\right) \text { for all } s_{i}^{\prime} \in D_{i} .
$$

Proof. Obviously, if there exist a $p_{i} \in \Delta\left(D_{-i}\right)$ and a $u_{i} \in U_{i}$ such that

$$
u_{i}\left(s_{i}, p_{i}\right) \geq u_{i}\left(s_{i}^{\prime}, p_{i}\right) \text { for all } s_{i}^{\prime} \in D_{i}
$$

then $s_{i}$ is not strictly dominated w.r.t. $U$ on $D_{i} \times D_{-i}$. Now, suppose that $s_{i}^{*} \in D_{i}$ is such that there is no $p_{i} \in \Delta\left(D_{-i}\right)$ and no
$u_{i} \in U_{i}$ such that

$$
u_{i}\left(s_{i}^{*}, p_{i}\right) \geq u_{i}\left(s_{i}^{\prime}, p_{i}\right) \text { for all } s_{i}^{\prime} \in D_{i} .
$$

Wr show that $s_{i}^{*}$ is strictly dominated w.r.t. $U$ on $D_{i} \times D_{-i}$. Every $v_{i} \in$ is closed under Harsanyi aggregation, for every over $\Delta\left(S_{i}\right)$. Since $V_{i}\left(U_{i}, \Delta\left(S_{-i}\right)\right)$ for every $\lambda \in[0,1]$ it holds that

$$
\lambda v_{i}^{1}+(1-\lambda) v_{i}^{2} \in V_{i}\left(U_{i}, \Delta\left(S_{-i}\right)\right) .
$$


We define the set

$$
\mathcal{U}:=\left\{\left(u_{i}\left(s_{i}, p_{i}\right)-u_{i}\left(s_{i}^{*}, p_{i}\right)\right)_{s_{i} \in S_{i}} \mid p_{i} \in \Delta\left(S_{-i}\right), u_{i} \in U_{i}\right\} .
$$

Claim The set $\mathcal{U}$ is convex and closed.

Proof of the Claim First we show convexity of $\mathcal{U}$. We must show that for all $\Pi^{1}, \Pi^{2} \in \mathcal{U}$ and for all $\lambda \in[0,1]$ it holds that

$$
\lambda \Pi^{1}+(1-\lambda) \sqcap^{2} \in \mathcal{U}
$$

Every element $\Pi$ of $\mathcal{U}$ can be written as

$$
\Pi=v_{i}\left(u_{i}, p_{i}\right)-\xi_{i}
$$

for some $v_{i}\left(u_{i}, p_{i}\right)$ where $\xi_{i}:=u_{i}\left(s_{i}^{*}, p_{i}\right)$ which is a constant for every fixed choice of $u_{i}, p_{i}$. Hence,

$$
\lambda \Pi^{1}+(1-\lambda) \Pi^{2}=\lambda v_{i}^{1}\left(u_{i}^{1}, p_{i}^{1}\right)-\xi_{i}^{1}+(1-\lambda)\left(v_{i}^{2}\left(u_{i}^{2}, p_{i}^{2}\right)-\xi_{i}^{2}\right)
$$

Therefore, convexity of $\mathcal{U}$ follows from closedness under Harsanyi aggregation and the fact that $V_{i}\left(U_{i}, \Delta\left(S_{-i}\right)\right)$ for all $v_{i}$ contains all positive affine transformations (thus $v_{i} \in V_{i}\left(U_{i}, \Delta\left(S_{-i}\right)\right)$ implies $v_{i}-\xi_{i} \in V_{i}\left(U_{i}, \Delta\left(S_{-i}\right)\right)$ ).

Closedness of $\mathcal{U}$ follows directly from closedness of $U_{i}$ and $\Delta\left(S_{-i}\right)$ for all $i \in I$.

We continue the main part of our proof by defining the set of negative vectors

$$
\mathcal{V}:=\left\{x \in \mathbb{R}^{S_{i}} \mid x \leq 0 \text { componentwise }\right\}
$$

Since. $s_{i}^{*}$ is never optimal for any $p_{i} \in \Delta\left(S_{-i}\right)$ and any $u_{i} \in U_{i}$ it holds that

$$
\mathcal{U} \cap \mathcal{V}=\varnothing \text {. }
$$

Therefore, by the separating hyperplane theorem, there exists a vector $y \geq 0 \in$ $\mathbb{R}^{S_{2}}$ with at least one positive component such that

$$
\begin{aligned}
& u \cdot y>0 \text { for all } u \in \mathcal{U} \\
& v \cdot y<0 \text { for all } v \in \mathcal{V} .
\end{aligned}
$$

Assume, namely, that there is an $k \in\left|S_{i}\right|$ such that $y_{k}<0$. Then we can make the component $v_{k}$, that is negative by definition, so small that $v \cdot y>0$, contradicting the Separating hyperplane Theorem.

Hence, there exists a normalized version of $y$, i.e. a $\mu_{i} \in \Delta\left(S_{i}\right)$ such that

$$
\mu_{i} \cdot u>0 \text { for all } u \in \mathcal{U},
$$

implying that the expected utility satisfies

$$
u_{i}\left(\mu_{i}, p_{i}\right)>u_{i}\left(s_{i}^{*}, p_{i}\right)
$$

for all $u_{i} \in U_{i}$ and for all $p_{i} \in \Delta\left(S_{-i}\right)$. This implies that $s_{i}^{*}$ is strictly dominated by $\mu_{i}$ w.r.t. $U$, which completes our proof. 
Example 3.18 (Example 3.3 revisited) Consider again $U_{1}=\operatorname{convh}\left\{u_{1}, u_{1}^{\prime}\right\}$ with

\begin{tabular}{|c|c|c|c|c|c|c|}
\hline $\mathbf{u}_{1}$ & $d$ & $e$ & & $\mathrm{u}_{1}^{\prime}$ & $d$ & $e$ \\
\hline$a$ & 2 & 0 & and & $a$ & 4 & -1 \\
\hline b) & -1 & 4 & & $b$ & 0 & 2 \\
\hline 0 & 1 & 1 & & $c$ & 1 & 1. \\
\hline
\end{tabular}

Assume that

$$
\begin{aligned}
& \mu_{2}=d \text { and } u_{1}=u \\
& \mu_{2}^{\prime}=e \text { and } u_{1}^{\prime}=u^{\prime} .
\end{aligned}
$$

For $\lambda \in(0.1)$ one can show that there are no $u_{1}^{*}, \mu_{2}^{*}$ such that

$$
\lambda v_{1}(u, d)+(1-\lambda) v_{1}\left(u^{\prime}, e\right)=v_{1}\left(u_{1}^{*}, \mu_{2}^{*}\right) .
$$

Hence, the set $V_{1}\left(U_{1}, \Delta\left(S_{2}\right)\right)$ is not convex and the set $\Lambda_{1}$ is not closed under Harsanyi aggregation (assume that $\mu_{2}=(1,0)$ and $\mu_{2}^{\prime}=(0,1)$. Then, $\left.v_{i}\left(u, \mu_{2}\right)+v_{\imath}\left(u^{\prime} \cdot \mu_{2}^{\prime}\right) \notin \Lambda_{i}\right)$.

\subsection{A special case}

\subsubsection{Cournot model of duopoly}

Consider two firms 1 and 2 that compete on a market and produce the same good. The firms choose quantities $q_{1}, q_{2}$, respectively and the market price $p$ is marginal costs. Five c $\bar{c}_{2}$ ind 2 marginal costs $c_{2}$ to be in the interval $\left[\underline{c}_{2}, \vec{c}_{2}\right]$ and. vice versa, firm 2 believes firm 1 's costs $c_{1}$ to be in the interval $\left[\underline{c}_{1}, \bar{c}_{1}\right]$. For $i=1.2$ the utility functions are given by

$$
\begin{aligned}
u_{i}\left(q_{1}, q_{2}\right) & =q_{i}\left(p-c_{i}\right) \\
& =q_{i}\left(a-q_{1}-q_{2}-c_{i}\right)
\end{aligned}
$$

Hence, we define the classes of utility functions

$$
\begin{aligned}
& U_{1}:=\left\{u_{1}\left(q_{1}, q_{2}\right) \mid u_{1}\left(q_{1}, q_{2}\right)=q_{1}\left(a-q_{1}-q_{2}-c_{1}\right) \text { for } c_{1} \in\left[c_{1}, \bar{c}_{1}\right]\right\} \\
& U_{2}:=\left\{u_{2}\left(q_{1}, q_{2}\right) \mid u_{1}\left(q_{1}, q_{2}\right)=q_{2}\left(a-q_{1}-q_{2}-c_{2}\right) \text { for } c_{2} \in\left[\underline{c}_{2}, \bar{c}_{2}\right]\right\}
\end{aligned}
$$

First we check whether the classes of utility functions that are induced by the uncertainty about the marginal costs and uncertainty about the opponent's utility function that is induced by marginal aggregation. Let $v_{2}\left(c_{2}, q_{1}\right)$ be the chooses $q_{1}$. Hence,

$$
v_{2}\left(c_{2}, q_{1}\right)\left(q_{2}\right):=q_{2}\left(a-q_{1}-q_{2}-c_{2}\right)
$$


Let $\left(q_{1}, c_{2}\right),\left(q_{1}^{\prime}, c_{2}^{\prime}\right)$ be given. Then

$$
\left(v_{2}\left(c_{2}, q_{1}\right)+v_{2}\left(c_{2}^{\prime}, q_{1}^{\prime}\right)\right)\left(q_{2}\right)=2 q_{2}\left(a-q_{2}-\frac{q_{1}+q_{1}^{\prime}}{2}-\frac{c_{2}+c_{2}^{\prime}}{2}\right)
$$

is induced by

$$
\begin{aligned}
& q_{1}^{\prime \prime}:=\frac{q_{1}+q_{1}^{\prime}}{2} \\
& c_{2}^{\prime \prime}:=\frac{c_{2}+c_{2}^{\prime}}{2} .
\end{aligned}
$$

A similar argument holds for firm 1 . Hence, the sets $V_{i}\left(U_{i}, \Delta\left(S_{-i}\right)\right)$ are closed under Harsanyi aggregation.

Next we answer the question which quantities can rationally be chosen under common belief in rationality. Theorem 3.17 implies that we can equivalently investigate which quantitios survive the iterative elimination of strictly dominated quantities. Therefore we first calculate the optimal quantities for the two frims, i.e. $q_{1}$ given $q_{2}$ and vice versa. If we want to calculate the optimal quantity $q_{1}^{*}$ given $q_{2}$, we calculate the derivative of $u_{1}$ with respect to $q_{1}$

$$
\frac{d u_{1}}{d q_{1}}\left(q_{1}, q_{2}\right)=a-q_{2}-c_{1}-2 q_{1},
$$

yielding an optimal value for $q_{1}$

$$
q_{1}^{*}\left(q_{2}\right)=\frac{a-q_{2}-c_{1}}{2}
$$

Analogously we derive the same expression for firm 2

$$
q_{2}^{*}\left(q_{1}\right)=\frac{a-q_{1}-c_{2}}{2}
$$

Next we describe the procedure of iterative elimination of strictly dominated strategies for this case. First consider firm 1. Assume that firm 1 believes that $q_{2}^{1}$ (indicating that we are in step 1 of the elimination process) is in $\left[\underline{q}_{2}^{1}, \bar{q}_{2}^{1}\right]$. Hence, by (3.2), the smallest $q_{1}^{1}$ that firm 1 can rationally choose given $q_{2}^{1} \in\left[\underline{q}_{2}^{1}, \tilde{q}_{2}^{1}\right]$ and $c_{1} \in\left[\underline{c}_{1}, \bar{c}_{1}\right]$ is given by

$$
\underline{q}_{1}^{1}=\frac{a-\bar{q}_{2}^{1}-\bar{c}_{1}}{2}
$$

Analogously, the largest $\bar{q}_{1}^{1}$ that can rationally be chosen by firm 1 under the same assumptions is

$$
\bar{q}_{1}^{1}=\frac{a-\underline{q}_{2}^{1}-\underline{c}_{1}}{2}
$$

Hence, we obtain

$$
\left[\underline{q}_{1}^{1}, \bar{q}_{1}^{1}\right]=\left[\frac{a-\bar{q}_{2}^{1}-\bar{c}_{1}}{2}, \frac{a-\underline{q}_{2}^{1}-\underline{c}_{1}}{2}\right] .
$$


Similarly we ohtain for firm 2

$$
\left[\underline{q}_{2}^{1}, \bar{q}_{2}^{1}\right]=\left[\frac{a-\bar{q}_{1}^{1}-\bar{c}_{2}}{2}, \frac{a-\underline{q}_{1}^{1}-\underline{c}_{2}}{2}\right] \text {. }
$$

In the serond step wo assime that firm 1 believes that firm 2 follows the same "liminatim procolure. Hence, firm 1 can substitute $\underline{q}_{2}^{1}, \bar{q}_{2}^{1}$ from (3.4) and obtains that the set of all $q_{1}^{2}$ that firm 1 can rationally choose under the assumption that firm 2 rhouses rationally is

$$
\left[\underline{q}_{1}^{2}, \ddot{q}_{1}^{2}\right]=\left[\frac{a-\frac{a-\underline{q}_{1}^{1}-\underline{c}_{2}}{2}-\bar{c}_{1}}{2}, \frac{a-\frac{a-\bar{q}_{1}^{1}-\bar{c}_{2}}{2}-\underline{c}_{1}}{2}\right] .
$$

Grufrally, assume that we are in step $k+1$ of the elimination process. Then, as a generalization of (3.3) and (3.4) the intervals of both firms that can rationally b" chosen under common belief in the opponent being rational of "degree" $k$ (with which we mean that every firm believes that the opponent is rational, holieves that the opponent believes that he is rational and so on until step $k$ ) are:

and

$$
\left[\underline{q}_{1}^{k+1}, \bar{q}_{1}^{k+1}\right]=\left[\frac{a-\bar{q}_{2}^{k}-\bar{c}_{1}}{2}, \frac{a-\underline{q}_{2}^{k}-\underline{c}_{1}}{2}\right]
$$

$$
\left[\underline{q}_{2}^{k+1}, \bar{q}_{2}^{k+1}\right]=\left[\frac{a-\bar{q}_{1}^{k}-\bar{c}_{2}}{2}, \frac{a-\underline{q}_{1}^{k}-\underline{c}_{2}}{2}\right] .
$$

Now step $k+1$ would yield for firm 1 by substitution of step $k$

$$
\begin{aligned}
{\left[\underline{q}_{1}^{k+2} \cdot q_{1}^{k+2}\right] } & =\left[\frac{a-\bar{q}_{2}^{k+1}-\bar{c}_{1}}{2}, \frac{a-\underline{q}_{2}^{k+1}-\underline{c}_{1}}{2}\right] \\
& =\left[\frac{a-\frac{a-\underline{q}_{1}^{k}-\underline{c}_{2}}{2}-\bar{c}_{1}}{2}, \frac{a-\frac{a-\bar{q}_{1}^{k}-\bar{c}_{2}}{2}-\underline{c}_{1}}{2}\right] \\
& =\left[\frac{1}{4}\left(a-2 \bar{c}_{1}+\underline{c}_{2}+\underline{q}_{1}^{k}\right), \frac{1}{4}\left(a-2 \underline{c}_{1}+\bar{c}_{2}+\bar{q}_{1}^{k}\right)\right] .
\end{aligned}
$$

For thr limit $k \rightarrow \infty$ we calculate the fixpoints by the equations

violiling

$$
\begin{aligned}
& \underline{q}_{1}=\frac{1}{4}\left(a-2 \bar{c}_{1}+\underline{c}_{2}+\underline{q}_{1}\right) \\
& \ddot{q}_{1}=\frac{1}{4}\left(a-2 \underline{c}_{1}+\bar{c}_{2}+\bar{q}_{1}\right),
\end{aligned}
$$

$$
\begin{aligned}
& \underline{q}_{1}=\frac{1}{3}\left(a-2 \bar{c}_{1}+\underline{c}_{2}\right) \\
& \ddot{q}_{1}=\frac{1}{3}\left(a-2 \underline{c}_{1}+\bar{c}_{2}\right) .
\end{aligned}
$$




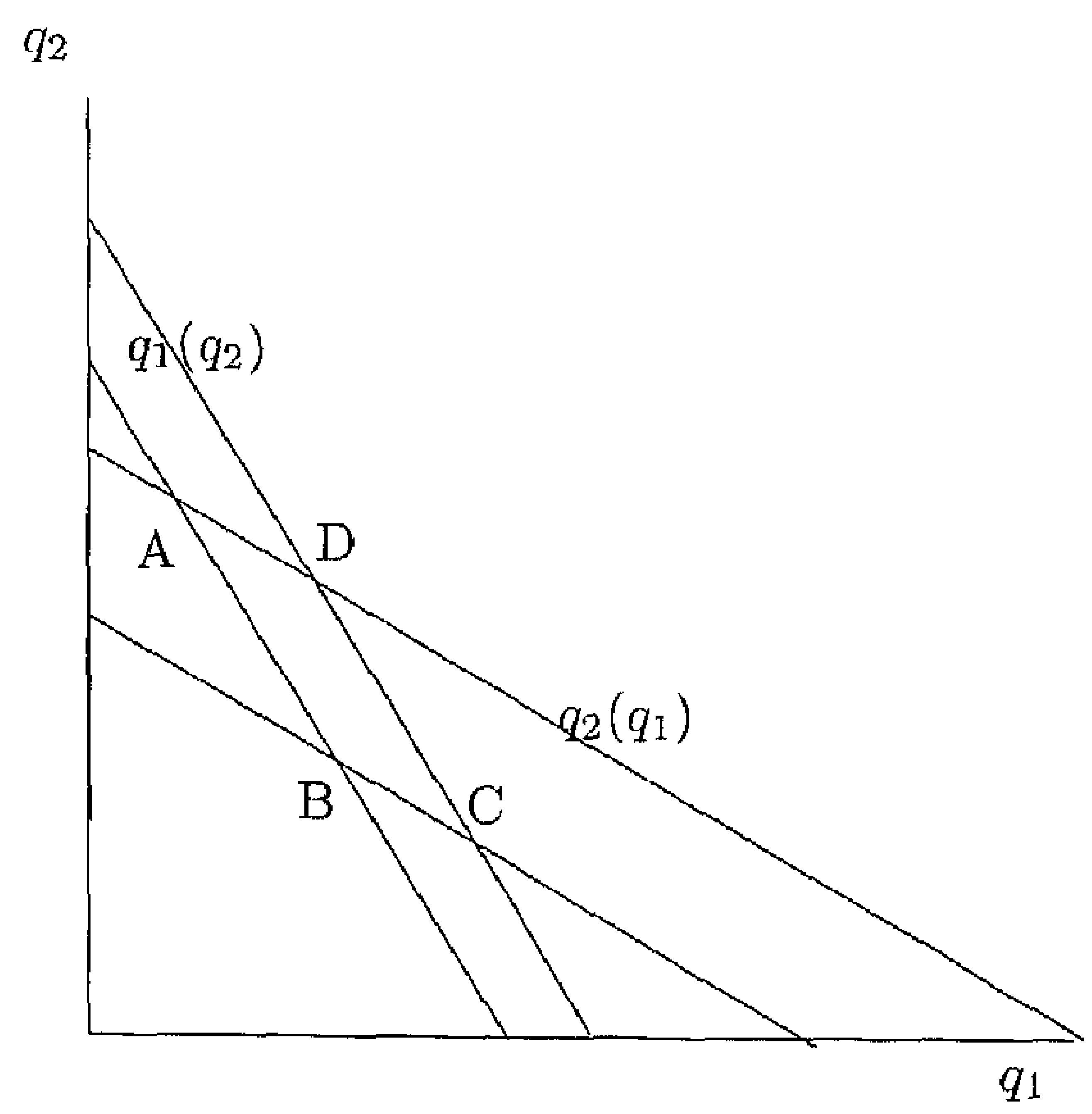

Figure 5

Hence, the intervals of quantities that can rationally be chosen under common belief in rationality are given by

$$
\begin{aligned}
& {\left[\underline{q}_{1}, \bar{q}_{1}\right]=\left[\frac{1}{3}\left(a-2 \bar{c}_{1}+\underline{c}_{2}\right), \frac{1}{3}\left(a-2 \underline{c}_{1}+\bar{c}_{2}\right)\right]} \\
& {\left[\underline{q}_{2}, \bar{q}_{2}\right]=\left[\frac{1}{3}\left(a-2 \bar{c}_{2}+\underline{c}_{1}\right), \frac{1}{3}\left(a-2 \underline{c}_{2}+\bar{c}_{1}\right)\right] .}
\end{aligned}
$$

The iterative process can intuitively also be seen in the following picture. In the first step we see from the graph that

$$
q_{1}^{1}<\frac{a-\underline{c}_{1}}{2}
$$

as derived above. Then, from the graph we see that

$$
q_{2}^{1}>\frac{a-\bar{q}_{1}^{1}-\bar{c}_{2}}{2}
$$

which is the intersection of the line $q_{1}=\frac{1}{2}\left(a-\underline{q}_{2}^{1}-\underline{c}_{1}\right)$ with the "lowest" graph $q_{2}\left(q_{1}\right)$ of firm 2 . Intersecting this line

$$
q_{2}=\frac{\left(a-\bar{q}_{1}^{1}-\bar{c}_{2}\right)}{2}=\frac{1}{2}\left(a-\frac{a-\underline{c}_{1}}{2}-\bar{c}_{2}\right)
$$


with the "highest" graph $q_{1}\left(q_{2}\right)$ of firm 1 we obtain

$$
q_{1}^{2}<\frac{a-q_{2}^{1}-\underline{c}_{1}}{2}=\frac{1}{2}\left(\frac{a-\underline{c}_{1}}{2}-\bar{c}_{2}-\underline{c}_{1}\right) .
$$

Iterating this process leads to the conclusion that the pairs of strategies that can rationally be chosen under common belief in rationality are in $A B C D$, which coincides with our conclusion above.

Next we compare this result to the "standard" formulation of the Cournot chopoly, i.e. the case in which there is no uncertainty about $c_{1}$ and $c_{2}$. If thror is no uncertainty we write

$$
\begin{aligned}
& c_{1}=\underline{c}_{1}=\bar{c}_{1} \\
& c_{1}=\underline{c}_{2}=\bar{c}_{2}
\end{aligned}
$$

and obtain

$$
\begin{aligned}
& q_{1}=\frac{1}{3}\left(a-2 c_{1}+c_{2}\right) \\
& q_{2}=\frac{1}{3}\left(a-2 c_{2}+c_{1}\right),
\end{aligned}
$$

which coincides with the solution of the standard Cournot duopoly.

Notice that if we assume that every firm has no uncertainty about its own marginal costs we obtain for the quantities every firm can rationally choose under common belief in rationality

$$
\begin{aligned}
& {\left[\underline{q}_{1}, \bar{q}_{1}\right]=\left[\frac{1}{3}\left(a-2 c_{1}+\underline{c}_{2}\right), \frac{1}{3}\left(a-2 c_{1}+\bar{c}_{2}\right)\right]} \\
& {\left[\underline{q}_{2}, \bar{q}_{2}\right]=\left[\frac{1}{3}\left(a-2 c_{2}+\underline{c}_{1}\right), \frac{1}{3}\left(a-2 c_{2}+\bar{c}_{1}\right)\right] .}
\end{aligned}
$$

Remark 3.19 As a further application of our main result from this chapter we can give a nice alternative proof of the pure strategy dominance theorem form [Börgers, 1993] if we succeed in proving closedness under Harsanyi aggregation for the corresponding set $V_{2}\left(U_{i}, \Delta\left(S_{-i}\right)\right)$. This, however, remained an open
problem to us. 


\section{Part II}

\section{Incomplete preferences}




\section{Chapter 4}

\section{Stochastic dominance equilibria in two-person games}

In this chapter we consider noncooperative games between players with incomplete preferences over lotteries. More precisely, we assume that they have complete preferences over certain alternatives, and incomplete preferences over lotteries resulting from playing mixed strategies.

An important motivation for considering incomplete preferences over lotteries is the intuition that completeness is a very strong assumption in many situations: it may be already quite demanding for players to be able to order all certain alternatives. It is not the objective of this monograph to delve deep into the discussion of (in)completeness of preferences. A recent paper that both presents a brief history of this discussion and investigates the formal consequences of dropping the completeness assumption from the von Neumann-Morgenstern conditions, is [Dubra et al, 2004]. In fact, their main result applies to the preferences we consider in this part of the dissertation (incomplete preferences).

To become more specific, we consider two-person games with finite (pure) strategy sets. The players have complete transitive asymmetric preferences (linear orderings) over the pure outcomes of the game, and evaluate probability distributions over the outcomes induced by mixed strategies by a stochastic dominance critcrion. This, of course, is a very specific way to deal with incomplete preferences, but there is an appropriate justification in terms of aversion to risk or, perhaps better, to bad outcomes, as we will see. With the exception of completeness of preferences, we do not deviate form the standard assumptions of noncooperative game theory, such as common knowledge between the players of the data of the game, including the (incomplete) preferences.

There is quite some literature on noncooperative games with only ordinal preferences: many economic games (for instance, Cournot or Bertrand oligopoly games) belong to this category, but also games used for implementing social 
choice correspondences, to name just a few examples. However, apart from [Fishburn, 1978] the only references to ordinal games with mixed strategies that we know of are [Börgers, 1993] and [Rothe, 1995]. Börgers proposes a definition of rationalizability in which only ordinal preferences over outcomes are assumed to be common knowledge. Rothe consiclers equilibrium selection in $2 \times 2$-games under the first-degree stochastic dominance critcrion.

According to first-degree stochastic dominance, probability distributions that shift more probability to better outcomes are considered more attractive. Since, indeed, the stochastic dominance ordering is not complete, we call a mixed strategy of a player a best reply against the strategy of the opponent if it induces an undominated probability distribution over the outcomes. An equilibrium is a pair of best replics. Fishburn established that the set of equilibria is equal to the union of all sets of Nash equilibria, taken over all possible utility representations of the preferences. This is an intuitive result in view of the familiar characterization of first-degree stochastic dominance which says that a distribution is undominated if and only if it maximizes expected utility for at least one utility representation of the preferences over certain outcomes.

We take this analysis a good deal further by assuming that the players' preferences over lotteries are more restricted or, equivalently, that the derived classes of utility functions which may represent their preferences are narrowed down. Specifically, we study so-called $t$-equilibria, where the natural number $t$ is the degree of stochastic dominance used to evaluate probability distributions. As is well-known, a distribution is second-degree stochastically undominated if and only if it maximizes expected utility for at least one concave utility representation of the preferences over certain outcomes. Loosely speaking, higher degrees of stochastic dominance correspond to higher degrees of risk aversion or, more specifically, higher degrees of aversion to bad outcomes. As the clegree of stochastic dominance becomes higher, preferences become more complete and, in the limit, would order lotteries by considering the worst outcomes.

\subsection{Stochastic dominance}

Let $\ell \geq 1$ be an integer and let $O=\{1, \ldots, \ell\}$ be a set of $\ell$ alternatives. For $1 \leq k<l \leq \ell$ we assume that a decision maker strictly prefers alternative $l$ to alternative $k$.

For a probability distribution $r=\left(r_{1}, \ldots, r_{\ell}\right)$ on $O$ (so each $l$ occurs with probability $\left.r_{l}\right)$ we define, recursively, for each $l \in\{1, \ldots, \ell\}, F_{r}^{0}(l)=r_{l}$ ancl

$$
F_{r}^{t}(l)=\sum_{i=1}^{l} F_{r}^{t-1}(i) \quad(t \geq 1) .
$$

So $F_{r}^{1}$ is the cumulative distribution function of $F_{r}^{0}=r$ and, similarly, $F_{r}^{t}$ 'accunulates' the weights assigned by $F_{r}^{t-1}$. For probability distributions $r$ and $s$ on $O, r$ t-th degree stochastically dominates $s$ if

$$
F_{r}^{t}(l) \leq F_{s}^{t}(l) \text { for every } l \in\{1, \ldots, \ell\} .
$$


Observe that at least one of these inequalities is strict if $r \neq s$. Hence, $t$-degree stochastic dominance is an asymmetric binary relation. It is easy to see that it is also reflexive and transitive but not complete. Clearly, it respects the presumed linear ordering on $O$.

For $t=1$, this relation means that $r$ puts more probability on better alternatives than $s$. It is well known that this is equivalent to the expected utility under $r$ being at least as large as the expected utility under $s$ for every utility representation of $\sigma$. For second degree stochastic dominance, an analogous equivalence holds if we restrict to concave utility functions, or, more generally, utility functions with non-increasing differences between adjacent alternatives. Note that $t$-th degree stochastic dominance implies $t+1$-th degree stochastic dominance. In a relative sense, a similar relation holds between $t+1$-th and $t$-th degree stochastic dominance as between second and first degree stochastic dominance. Thus, higher degree stochastic dominance can be associated with increased risk aversion of decision makers, who put increasing weight on bad outcomes when evaluating probability distributions.

Fishburn $(1976,1980)$ characterizes stochastic dominance in terms of utility functions and in terms of moments of distributions. Below, we provide a characterization of stochastic dominance in terms of utility functions that will be important for the remaincler of this dissertation. In a later chapter the result will be generalized to real values of the parameter $t$.

Denote $F_{r}^{t}=\left(F_{r}^{t}(1), \ldots, F_{r}^{t}(\ell)\right.$ and let $A=\left[a_{i j}\right]$ be the $\ell \times \ell$-matrix with

$$
a_{i j}=\left\{\begin{array}{ll}
1 & \text { if } i \leq j \\
0 & \text { if } i>j
\end{array} \text { for all } i, j \in\{1, \ldots, \ell\} .\right.
$$

Write $A^{t}=A \cdot A \cdot \ldots \cdot A(t$ times, $t \in \mathbb{N})$. The following lemma gives a convenient representation of $F_{r}^{t}$.

Lemma 4.1 $F_{r}^{t}=r A^{t}$ for every $t \in \mathbb{N}$.

Proof. The proof is by induction on $t$. For $t=1$ the identity in the lemma holds by definition. Assume it holds for every $k<t(t \geq 2)$. Let $l \in\{1, \ldots, \ell\}$. Then

$$
F_{r}^{t}=F_{r}^{t-1} A=r A^{t-1} A=r A^{t},
$$

where the sccond equality follows by induction.

Denoting the element in row $i$ and column $j$ of $A^{t}$ by $a_{i j}^{t}$, we derive the following expression for this number.

Lemma 4.2 Let $t \in \mathbb{N}$. Then

$$
a_{i j}^{t}= \begin{cases}\frac{(j-i+t-1) !}{(j-i) !(t-1) !} & \text { if } i \leq j \\ 0 & \text { if } i>j\end{cases}
$$

Proof. The proof is by induction on $t$. For $t=1$ the formula holds by definition of $A$. Let the formula be true for all $k<t$, where $t \geq 2$. Then for all 
$i, j \in\{1, \ldots, \ell\}$

$$
a_{i j}^{t}=\sum_{l=1}^{\ell} a_{i l}^{t-1} a_{l j}=\sum_{l=i}^{\ell} a_{i l}^{t-1} a_{l j}
$$

where the second equality holds by induction. If $i>j$ then every $a_{l j}=0$ in the RHS of (4.1) since $l>j$. If $i \leq j$ then (4.1) implies by induction

$$
a_{i j}^{t}=\sum_{l=i}^{j} a_{i l}^{t-1}=\sum_{l=i}^{j} \frac{(t-2+l-i) !}{(l-i) !(t-2) !} .
$$

We are done if we can prove

$$
\sum_{l=i}^{j} \frac{(t-2+l-i) !}{(l-i) !(t-2) !}=\frac{(t-1+j-i) !}{(j-i) !(t-1) !} .
$$

We show this again by induction. For $j=i$ it is immediate. Let the equality hold for $i, \ldots, j-1$, then

$$
\begin{aligned}
\sum_{l=i}^{j} \frac{(t-2+l-i) !}{(l-i) !(t-2) !} & =\frac{(t-1+j-1-i) !}{(j-1-i) !(t-1) !}+\frac{(t-2+j-i) !}{(j-i) !(t-2) !} \\
& =\frac{(t-1+j-i-1) !(j-i)+(t-2+j-i) !(t-1)}{(j-i) !(t-1) !} \\
& =\frac{(t-1+j-i) !}{(j-i) !(t-1) !} .
\end{aligned}
$$

This completes the proof.

The following lemma applies to $t$ approaching infinity.

Lemma 4.3 Let $i, i^{\prime}, j \in\{1, \ldots, \ell\}$ with $i<i^{\prime} \leq j$. Then $a_{i j}^{t} \geq a_{i^{\prime} j}^{t}$ for every $t \in \mathbb{N}$ and $\lim _{t \rightarrow \infty} a_{i j}^{t} / a_{i^{\prime} j}^{t}=\infty$.

Proof. By Lemma 4.2,

$$
\begin{aligned}
a_{i j}^{t} / a_{i^{\prime} j}^{t} & =\frac{(t-1+j-i) !}{(j-i) !(t-1) !} \cdot \frac{\left(j-i^{\prime}\right) !(t-1) !}{\left(t-1+j-i^{\prime}\right) !} \\
& =\frac{(j-i+1) \cdot(j-i+2) \cdots(j-i+t-1)}{\left(j-i^{\prime}+1\right) \cdot\left(j-i^{\prime}+2\right) \cdots\left(j-i^{\prime}+t-1\right)} \\
& =\frac{\left(j-i^{\prime}+t\right) \cdot\left(j-i^{\prime}+t+1\right) \cdots(j-i+t-1)}{\left.\left(j-i^{\prime}+1\right) \cdot\left(j-i^{\prime}+2\right) \cdots(j-i)\right)},
\end{aligned}
$$

hence $a_{i j}^{t} \geq a_{i^{\prime} j}^{t}$ and $\lim _{t \rightarrow \infty} a_{i j}^{t} / a_{i^{\prime} j}^{t}=\infty$.

Lemma 4.4 Let a probability distribution $r$ on $O$ be $(t+1)$-undominated. Then $r$ is $t$-undominated. 
Proof. By assumption, for all probability distributions $s$ there exists an $l \in\{1, \ldots, \ell\}$ such that

$$
F_{r}^{t+1}(l)<F_{s}^{t+1}(l)
$$

By definition we obtain

$$
\sum_{i=1}^{l} F_{r}^{t}(i)<\sum_{i=1}^{l} F_{s}^{t}(i)
$$

which implies that there exists an $i^{\prime} \in\{1, \ldots, l\}$ such that

$$
F_{r}^{t}\left(i^{\prime}\right)<F_{s}^{t}\left(i^{\prime}\right)
$$

Hence, $r$ is $t$-undominated.

For every $t \in \mathbb{N}$ let $^{1}$

$$
U^{l}:=\left\{u \in \mathbb{R}^{\ell} \mid u=-A^{t} c \text { for some } c \in \mathbb{R}, c>0\right\}
$$

and

$$
\bar{U}^{t}:=\left\{u \in \mathbb{R}^{\ell} \mid u=-A^{t} c \text { for some } c \in \mathbb{R}, c \geq 0\right\} .
$$

An element $u$ of $U^{t}$ can be interpreted as a utility function representing the linear ordering on $O$ by the assignment $i \mapsto u_{i}$, since $u_{1}<u_{2}<\cdots<u_{\ell}$. The set $U^{t}$ is particularly relevant in Section 4.4 when we charactcrize and prove the existence of $t$-equilibria. Note that $U^{1}$ contains essentially any utility representation of $\sigma$. This is consistent with remarks made earlier. The set $U^{t}$ is decreasing in $t$.

The set $\bar{U}^{t}$ is a convex and closed set (the topological closure of $U^{t}$ ). Using Lemma 4.1, it is straightforward to derive the following proposition.

Proposition 4.5 For all probability distributions $r$ and $s$ on $O, r$-degree stochastically dominates $s$ if and only if $\sum_{l=1}^{\ell} r_{l} u_{l} \geq \sum_{l=1}^{\ell} s_{l} u_{l}$ for all $u \in \bar{U}^{t}$.

This result adapts Fishburn (1976) to our context, and is a special case of the main theorem in Dubra et al. (2004). Note that Proposition 4.5 would remain true if we replace $\vec{U}^{t}$ by $U^{t}$.

\subsection{Two-person games and $t$-equilibria}

Consicler two players. Player 1 has pure strategy set $M=\{1, \ldots, m\}$. A (mixed) strategy for player 1 is a probability distribution over $M$. Denote the set of strategies for player 1 by $\Delta^{M}$. A pure strategy $i$ is identified with the mixed strategy $e^{i} \in \Delta^{M}$, where $e_{k}^{i}=1$ if $k=i$ and $e_{k}^{i}=0$ otherwise. Similarly, player 2 has pure strategy set $N=\{1, \ldots, n\}$ and (mixed) strategy set $\Delta^{N}$. A pure strategy $j$ is identified with the mixed strategy $e^{j} \in \Delta^{N}$. If player 1 plays pure strategy $i$ and player 2 pure strategy $j$, then the alternative

\footnotetext{
${ }^{1}$ For vectors $x$ and $y, x>y[x \geq y]$ means $x_{i}>y_{i}\left[x_{i} \geq y_{i}\right]$ for every coorclinate $i$. Similarly for $x<y, x \leq y$.
} 
$o_{i j}$ results. If player 1 plays $p \in \Delta^{M}$ and player 2 plays $q \in \Delta^{N}$, then $o_{i j}$ results with probability $p_{i} q_{j}$. Let $O:=\left\{o_{i j} \mid i \in M, j \in N\right\}$ and assume that players 1 and 2 have preference relations represented, respectively, by bijections $\sigma . \tau: M \times N \rightarrow\{1, \ldots, m n\}$. For instance, if $1 \leq k<l \leq m n$ then player 1 strictly prefers $o_{i j}$ to $o_{i^{\prime} j^{\prime}}$, where $\sigma(i, j)=l$ and $\sigma\left(i^{\prime}, j^{\prime}\right)=k$.

For $p \in \Delta^{M}$ and $q \in \Delta^{N}$ we denote by $p q \sigma$ the vector of probabilities with l-th coordinate $p q \sigma_{l}=p_{i} q_{j}$ such that $\sigma(i, j)=l$, for all $l \in\{1, \ldots, m n\}$. We assume that the playcrs evaluate strategies according to a stochastic dominance criterion. More precisely, let $t \in \mathbb{N}$ and fix a strategy $q \in \Delta^{N}$ for player 2 . Then a strategy $p \in \Delta^{M I}$ of player 1 results in the weight vector $F_{p q \sigma}^{t}$, which depends on $\sigma$ and assigns weight $F_{p q \sigma}^{t}(\sigma(i, j))$ to alternative $o_{i j}$.

We call $p$ a t-best reply against $q$ if there is no $p^{\prime} \in \Delta^{M}$ such that $p^{\prime} q \neq p q$ and $p^{\prime} q t$-th degree stochastically dominates $p q$. The definition of a $t$-best reply $q$ against $p$ is analogous. A pair $(p, q) \in \Delta^{M} \times \Delta^{N}$ is a t-equilibrium if $p$ is a $t$-best reply against $q$ and vice versa. By $E^{t}$ we denote the set of $t$-equilibria.

\subsection{An example}

The example presented here is illustrative of the main results of this chapter, namely

(i) existence and characterization of $t$-equilibria and

(ii) asymptotic behavior for $t$ approaching infinity.

Let $m=n=2$ and consider the game

$$
\left[\begin{array}{ll}
o_{11} & o_{12} \\
o_{21} & o_{22}
\end{array}\right]
$$

where the rows are the pure strategies of player 1 , the columns those of player 2 , and the preferences are given by $\sigma(1,2)=1, \sigma(2,2)=2, \sigma(2,1)=3$, and $\sigma(1,1)=4$ for player 1 and $\tau(1,2)=1, \tau(1,1)=2, \tau(2,1)=3$, and $\tau(2,2)=4$ for player 2 .

We concentrate on player 1 . The matrix $A^{t}(t \geq 1)$ can be computed using Lemma 4.2. This results in

$$
A^{t}=\left[\begin{array}{cccc}
1 & t & \frac{1}{2} t(t+1) & \frac{1}{6} t(t+1)(t+2) \\
0 & 1 & t & \frac{1}{2} t(t+1) \\
0 & 0 & 1 & t \\
0 & 0 & 0 & 1
\end{array}\right]
$$

Consider strategies $p=\left(p_{1}, p_{2}\right)$ and $q=\left(q_{1}, q_{2}\right)$ for players 1 and 2 , respectively. In order to examine $t$-best replies of player 1 we compute (cf. Lemma 4.1)

$$
F_{p q \sigma}^{t}=\left(p_{1} q_{2}, p_{2} q_{2}, p_{2} q_{1}, p_{1} q_{1}\right) A^{t}
$$


Dropping the part that does not depend on $p$ and which therefore is not needed to compute $t$-best replies of player 1 , this results in the vector

$$
p_{1}\left(q_{2},(t-1) q_{2}, \frac{1}{2}\left(t^{2}-t\right) q_{2}-q_{1}, \frac{1}{6}\left(t^{3}-t\right) q_{2}-q_{1}(t-1)\right)
$$

For $t=1,(4.3)$ reduces to $p_{1}\left(q_{2}, 0,-q_{1}, 0\right)$. Since player 1 wants to 'minimizc" this vector, the $t$-best responses are $p_{1}=0$ if $q_{1}=0, p_{1}=1$ if $q_{1}=1$, and any $0 \leq p_{1} \leq 1$ if $0<q_{1}<1$. With a similar argument for player 2 (not reproduced here) we find the set $E^{1}$ : it contains the two pure Nash equilibria of the game, resulting in $o_{11}$ and in $o_{22}$, and all strategy combinations where no player plays a pure strategy. This is no surprise: in general, $E^{1}$ consists of all strategy combinations that are a Nash equilibrium for at least one choice of utility functions representing $\sigma$ and $\tau$. This is a consequence of the familiar characterization of first degree stochastic dominance using utility representations, mentioned in Section 2. See also Fishburn (1978), where the result is derived formally.

Next, consider $t=2$, so best replies are second degree stochastically undominated. By substituting $t=2$ in (4.3) it follows that for $q_{1}=1$ the 2-best reply is $p_{1}=1$, for $1>q_{1}>\frac{1}{2}$ any $0 \leq p_{1} \leq 1$ is a 2-best reply, and for $q_{1} \leq \frac{1}{2}$ the 2 -best reply is $p_{1}=0$. Again after a similar argument for player 2 it follows that $E^{2}$ consists of the two pure Nash equilibria of the game plus the set

$$
\left\{(p, q) \mid \frac{1}{2}<q_{1}<1,0<p_{1}<\frac{1}{2}\right\} .
$$

In these mixed strategy equilibria player 1 puts a larger weight on row 2. Row 2 is player 1's max-min pure strategy: he prefers the worst alternative in row $2, o_{22}$, to the worst alternative in row $1, o_{12}$. Thus, one might be tempted to conclude that a higher $t$ leads to max-min play. This, however, is deceptive. As will turn out later, what is important is that row 2 is the max-min row from the point of view of player 2: player 2 prefers the worst alternative (for him) of row $2, o_{21}$, to the worst alternative of row $1, o_{12}$. (A similar consideration holds for the strategy of player 2.)

Observe also that the 2-best reply correspondences of the players are not upper semi-continuous (their graphs are not closed).

For $t>2$, let $\hat{q}_{1}=\left(t^{3}-t\right) /\left(t^{3}+5 t-6\right)$. For $0 \leq q_{1} \leq \hat{q}_{1}$ the $t$-best reply is $p_{1}=0$, for $\hat{q}_{1}<q_{1}<1$ any $p_{1}$ is a $t$-best reply, and for $q_{\perp}=1$ the $t$-best reply is $p_{1}=1$. The $t$-equilibria are again the two pure Nash equilibria in the game together with the collection

$$
\left\{(p, q) \mid \frac{t^{3}-t}{t^{3}+5 t-6}<q_{1}<1,0<p_{1}<1-\frac{t^{3}-t}{t^{3}+5 t-6}\right\}
$$

For $t \rightarrow \infty$ these mixed strategy $t$-equilibria converge to the pure strategy combination of row 2 and column 1 . 


\subsection{Existence and characterization of $t$-equilibria}

The existence of $t$-equilibria can be proved directly by applying a fixed point argument to the best-reply correspondences. This proof is not completely straightforward since the best reply correspondences do not have to be upper semicontinuous, see the example in the previous section, so that the argument has to be applied to a suitable sub-correspondence.

We provide an existence proof of $t$-equilibria by applying the Kakutani fixed point theorem to subsets of the $t$-best reply correspondences.

Consider the two-person game defined in Section 4.2. Fix a strategy $q \in \Delta^{n}$ for player 2. By Lemma 4.1 it follows that

$$
F_{p q \sigma}^{t}=\sum_{i=1}^{m} p_{i} F_{e^{2} q \sigma}^{t}
$$

for every $p \in \Delta^{m}$. Hence

$$
\left\{F_{p q \sigma}^{t} \mid p \in \Delta^{m}\right\}=\operatorname{conv}\left\{F_{e^{i} q \sigma}^{t} \mid i \in M\right\},
$$

where 'conv' denotes 'the convex hull of'. The $t$-best replies against $q$ are those strategies $p$ that generate points on the 'southwest corner' of the polytope in (4.5), i.e., each point for which there is no other point in the polytope that has every coordinate smaller or equal and, thus, $t$-degree stochastically dominates it. These undominated points do not necessarily lie in the same face of the polytope and therefore a convex combination of $t$-best replies against $q$ is not necessarily a $t$-best reply itself against $q$. So $t$-best rcply correspondences are not necessarily convex-valued, in contrast with Nash best rcply corresponclences. For applying a fixed point argument this lack of convexity is not a real problem since from (4.5) it is easily seen that the set of $t$-best replies is homeomorphic to a convex set. In the proof of the existence result we apply Kakutani to the correspondences obtained by minimizing sums of coordinates on the polytopes in (4.5).

Theorem $4.6 E^{t} \neq \emptyset$ for every $t \geq 1$.

Proof. Let $t \geq 1$. For every $q \in \Delta^{n}$ define

$$
\mu_{1}(q)=\arg \min \left\{\sum_{l=1}^{m n} F_{p q \sigma}^{t}(l) \mid p \in \Delta^{m}\right\} .
$$

Then it is easy to verify that the correspondence $q \mapsto \mu_{1}(q)$ is nonempty- and convex-valued, and upper semi-continuous. For player 2 , we define an analogous correspondence $\Delta^{m} \ni p \mapsto \mu_{2}(p)$ with the same properties. Now the correspondence

$$
\mu: \Delta^{m} \times \Delta^{n} \ni(p, q) \mapsto \mu_{1}(q) \times \mu_{2}(p)
$$

satisfies all conditions needed to apply the Kakutani fixed point theorem. Hence, there exists a pair $\left(p^{*}, q^{*}\right)$ with $p^{*} \in \mu_{1}\left(q^{*}\right)$ and $q^{*} \in \mu_{2}\left(p^{*}\right)$. Since, for any 
$p$ and $q$, all elements of $\mu_{1}(q)$ are $t$-best replies against $q$ and all elements of $\mu_{2}(p)$ are $t$-best replies against $p$, this holds in particular for $p^{*}$ and $q^{*}$. Hence, $\left(p^{*}, q^{*}\right) \in E^{t}$.

Alternatively, $t$-equilibria can be characterized as Nash equilibria for suitably chosen utility functions. Existence then follows from the standard existence result for Nash equilibrium. For $t=1$, this has already been done in [Fishburn, 1978]. As a generalization of this result we give an alternative proof that uses the representation of a stochastic dominance preference relation by classes $U^{t}$.

In the next lemma we consider the game as defined in Section 4.2. The bijection $\sigma$ represents the preference relation of player 1. The set $U^{t}$ was defined in Section 4.1 .

Lemma 4.7 Let $p \in \Delta^{M}, q \in \Delta^{N}$, and $t \geq 1$. Then $p$ is a $t$-best reply against $q$ if and only if there is a $u^{t} \in U^{t}$ such that

$$
\sum_{i=1}^{m} \sum_{j=1}^{n} p_{i} q_{j} u_{\sigma(i, j)}^{t} \geq \sum_{i=1}^{m} \sum_{j=1}^{n} p_{i}^{\prime} q_{j} u_{\sigma(i, j)}^{t}
$$

for all $p^{\prime} \in \Delta^{M I}$.

Proof. $p$ is a $t$-best reply against $q$ if and only if there is no $p^{\prime} \in \Delta^{M}$ such that $F_{p^{\prime} q \sigma}^{t} \leq F_{p q \sigma}^{t}$ with at least one coordinate strictly smaller. This is the case if and only if

$$
\left\{x \in \mathbb{R}^{m n} \mid x \leq F_{p q \sigma}^{t}\right\} \cap\left\{x \in \mathbb{R}^{m n} \mid x=F_{p^{\prime} q \sigma}^{t} \text { for some } p^{\prime} \in \Delta^{M}\right\}=\left\{F_{p q \sigma}^{t}\right\} .
$$

By a standard separation argument it follows that the two sets on the left-hand side of this identity can be separated by a hyperplane through $F_{p q \sigma}^{t}$ with a nonnegative normal $c \in R^{m n}$ such that $F_{p q \sigma}^{t} \cdot c \leq F_{p^{\prime} q \sigma}^{t} \cdot c$ for all $p^{\prime} \in \Delta^{M}$. Since the second set is a polytope, this normal can be chosen positive (see Shapley, 1959 , for a detailed argument). By Lemma 4.1 this is equivalent to $(p q \sigma) A^{t} c \leq$ $\left(p^{\prime} q \sigma\right) A^{t} c$ for all $p^{\prime} \in \Delta^{M}$. The proof is complete by taking $u^{t}:=-A^{t} c$.

Lemma 4.7 can be formulated for player 2 in an analogous way. Then $t$-equilibria can be characterized as follows.

Corollary 4.8 Let $\sigma$ and $\tau$ represent the preferences of players 1 and 2 , respectively. Let $t \geq 1, p^{*} \in \Delta^{M}$, and $q^{*} \in \Delta^{N}$. Then $\left(p^{*}, q^{*}\right)$ is a t-equilibrium if and only if there are $u, v \in U^{t}$ such that $\left(p^{*}, q^{*}\right)$ is a Nash equilibrium for the payoff functions $O \rightarrow \mathbb{R}$ defined by $o_{i j} \mapsto u_{\sigma(i, j)}$ and $o_{i j} \mapsto v_{\tau(i, j)}$ for playcrs 1 and 2 , respectively. Especially, the set of t-equilibria is equal to the union of Nash equilibria over all representing utility functions.

Since Nash equilibria always exist, Corollary 4.8 implies existence of $t$-equilibria.

Corollary $4.9 E^{t} \neq \emptyset$ for every $t \geq 1$. 


\subsection{Limit behavior of $t$-equilibria}

The example in Scction 4.3 suggests some kind of max-min behavior of the players in a $t$-equilibrium for $t$ going to infinity. In this section we consider this in detail. The setting is the general game model as defined in Section 4.2. Unless stated otherwise, the number $l$ is arbitrary but fixed. The preferences of the players are represented by the bijections $\sigma$ for player 1 and $\tau$ for player 2 . For $p \in \Delta^{M}$, the support of $p$ is the set

$$
\operatorname{supp}(p)=\left\{i \in M \mid p_{i}>0\right\}
$$

For $q \in \Delta^{N}, \operatorname{supp}(q)$ is defined in the same way. We start with an auxiliary result.

Lemma 4.10 Let $p^{t} \in \Delta^{M}$ and let $q^{t} \in \Delta^{N}$. Let $p \in \Delta^{M}$ and $q \in \Delta^{N}$ with $\operatorname{supp}(p) \subseteq \operatorname{supp}\left(p^{t}\right)$ and $\operatorname{supp}(q) \subseteq \operatorname{supp}\left(q^{t}\right)$. Then

(i) if $p^{t}$ is a t-best reply against $q^{t}$, then $p$ is a t-best reply against $q^{t}$;

(ii) if $q^{t}$ is a t-best reply against $p^{t}$, then $q$ is a t-best reply against $p^{t}$.

Proof. Apply Lemma 4.7.

Lemma 4.11 Let $I \subseteq M, J \subseteq N$, and let $\left(p^{t}, q^{t}\right)_{t \in \mathbb{N}}$ be a sequence of pairs of mixed strategies such that $I=\operatorname{supp}\left(p^{t}\right)$ and $J=\operatorname{supp}\left(q^{t}\right)$ for all $t \in \mathbb{N}$.

(i) Let $p^{t}$ be a t-best reply against $q^{l}$ for every $t \in \mathbb{N}$. Then, for every $i \in M$, there is a $j \in J$ such that $\sigma(i, j)<\sigma\left(i^{\prime}, j\right)$ for all $i^{\prime} \in I \backslash\{i\}$.

(ii) Let $q^{t}$ be a $t$-best reply against $p^{t}$ for every $t \in \mathbb{N} N$. Then, for every $j \in N$, there $i$ an $i \in I$ such that $\tau(i, j)<\tau\left(i, j^{\prime}\right)$ for all $j^{\prime} \in J \backslash\{j\}$.

Proof. We only prove (i), the proof of (ii) is analogous. Suppose (i) were not true. Then there is an $\hat{\imath} \in M$ such that for every $j \in J$

$$
I_{j}:=\{i \in I \mid \sigma(i, j)<\sigma(\hat{\imath}, j)\} \neq \emptyset \text {. }
$$

For every $j \in J$, choose an $i_{j} \in I_{j}$. Let $\hat{I}:=\left\{i_{j} \mid j \in J\right\}, s:=|\hat{I}|,{ }^{2}$ and define $\hat{p} \in \Delta^{M}$ by $\hat{p}_{i}=1 / s$ if $i \in \hat{I}$ and $\hat{p}_{i}=0$ otherwise. We will show that, for $t$ sufficiently large,

$$
F_{e^{i} q^{t} \sigma}^{t} \leq F_{\hat{p} q^{\prime} \sigma}^{t}
$$

Since, clearly, the two probability distributions $e^{\hat{\imath}} q^{t} \sigma$ and $\hat{p} q^{t} \sigma$ in (4.6) are different, this means that at least one of the inequalities must be strict if (4.6) holds. Since $\operatorname{supp}(\hat{p}) \subseteq I=\operatorname{supp}\left(p^{t}\right)$ for all $t,(4.6)$ contradicts Lemma 4.10. rThis proves (i).

\footnotetext{
${ }^{2}|\cdot|$ denotes the carclinality of a set.
} 
We are left to prove (4.6), hence we are left to prove

$$
F_{e^{i} q^{t} \sigma}^{t}(k) \leq F_{\tilde{p} q^{t} \sigma}^{t}(k) \text { for all } k=1, \ldots, m n \text {. }
$$

Fix $k \in\{1, \ldots, m n\}$ and define $J_{k}:=\{j \in J \mid \sigma(\hat{\imath}, j) \leq k\}$. Then

$$
\begin{aligned}
F_{e^{i} q^{t} \sigma}^{t}(k) & =\sum_{l=1}^{m n}\left(e^{\hat{\imath}} q^{t} \sigma\right)_{l} a_{l k}^{t} \\
& =\sum_{l=1}^{k}\left(e^{\hat{\imath}} q^{t} \sigma\right)_{l} a_{l k}^{t} \\
& =\sum_{j \in J_{k}} q_{j}^{t} a_{\sigma(\hat{\imath}, j), k}^{t}
\end{aligned}
$$

Here, the first equality follows from Lemma 4.1, the second equality from Lemma 4.2 , and the last equality by Lemma 4.2 and the definition of $J_{k}$.

Now

$$
\begin{aligned}
F_{\hat{p} q^{t} \sigma}^{t}(k) & =\sum_{l=1}^{k}\left(\hat{p} q^{t} \sigma\right)_{l} a_{l k}^{t} \\
& \geq \sum_{j \in J_{k}} q_{j}^{t} \hat{p}_{i_{j}} a_{\sigma\left(i_{j}, j\right), k}^{t} \\
& =\sum_{j \in J_{k}} \frac{1}{s} q_{j}^{t} a_{\sigma\left(i_{j}, j\right), k}^{t} \\
& \geq \sum_{j \in J_{k}} \frac{1}{s} q_{j}^{t} a_{\sigma(\hat{\imath}, j)-1, k}^{t}
\end{aligned}
$$

The first equality follows again by Lemmas 4.1 and 4.2 . The first inequality follows since some terms are left out. The second equality follows by definition of $\hat{p}$ since $i_{j} \in \hat{I}$ for every $j \in J$. The last inequality follows by the first statement in Lemma 4.3 since $\sigma\left(i_{j}, j\right) \leq \sigma(\hat{\imath}, j)-1$ for every $j \in J$.

If $J_{k}=\emptyset$ then (4.7) follows immediately from (4.8) and (4.9). Otherwise, by the second statement in Lomma 4.3 there is a $t$ sufficiently large such that for every $k=1,2, \ldots, m n$ and $j \in J_{k}$ we have

$$
\frac{1}{s} q_{j}^{t} a_{\sigma(\hat{\imath}, j)-1, k}^{t} \geq q_{j}^{t} a_{\sigma(\hat{\imath}, j), k}^{t} .
$$

Then (4.7) follows from (4.8), (4.9), and (4.10).

Lemma 4.11(i) implies that for every row (pure strategy) $i$ in $I$ there must be a column (pure strategy) $j$ in $J$ such that the resulting outcome $o_{i j}$ is the worst outcome for player 1 in that column restricted to the rows in $I$. In turn, this implies $|I| \leq|J|$. Similarly, Lemma 4.11(ii) implies $|J| \leq|I|$. So we have the following result. 
Corollary 4.12 Let $I \subseteq M, J \subseteq N$, and let $\left(p^{t}, q^{t}\right)_{t \in \mathbb{N}}$ be a sequence of $t$ equilibria such that $I=\operatorname{supp}\left(p^{t}\right)$ and $J=\operatorname{supp}\left(q^{t}\right)$ for all $t \in \mathbb{N}$. Then $|I|=|J|$.

If $|I|=|J|=1$ in Corollary 4.12, then the sequence of $t$-equilibria reduces to the constant Nash equilibrium in which player 1 picks the best element from the column played by player 2 and player 2 picks the best element from the row played by player 1 .

The next result implies that in Corollary 4.12 the $t$-equilibria must converge to pure strategy combinations.

Lemma 4.13 Let $I \subseteq M, J \subseteq N$, and let $\left(p^{t}, q^{t}\right)_{t \in \mathbb{N}}$ be a sequence of $t$ equilibria such that $I=\operatorname{supp}\left(p^{t}\right)$ and $J=\operatorname{supp}\left(q^{t}\right)$ for all $t \in \mathbb{N}$. Let $\hat{\imath} \in I$ and $\hat{\jmath} \in J$ be such that $\min \{\tau(i, j) \mid j \in J\}<\min \{\tau(\hat{\imath}, j) \mid j \in J\}$ for all $i \in I \backslash\{\hat{\imath}\}$ and $\min \{\sigma(i, j) \mid i \in I\}<\min \{\sigma(i, \hat{\jmath}) \mid i \in I\}$ for all $j \in J \backslash\{\hat{\jmath}\}$. Then (i) $\lim _{t \rightarrow \infty} p_{i}^{t}=1$ and (ii) $\lim _{t \rightarrow \infty} q_{j}^{t}=1$.

Proof. We only prove (ii), the proof of (i) is analogous. By Lemma 4.11 and Corollary 4.12 we may renumber the strategies of the players such that

a. $I=J=\{1, \ldots, s\}$ for some $s \geq 1$;

b. $\sigma(j, j)<\sigma(i, j)$ for every $j \in J$ and $i \in I \backslash\{j\}$;

c. $\sigma(1,1)<\sigma(2,2)<\ldots<\sigma(s, s)$.

Note that $\hat{\jmath}=s$. Let $s^{\prime} \in\{1, \ldots, s-1\}$ arbitrary. To prove (ii), it is sufficient to prove that $\lim _{t \rightarrow \infty} q_{s^{\prime}}^{t}=0$. Suppose that this is not the case. Then we may assume that there is an $\alpha>0$ such that $q_{s^{\prime}}^{l} \geq \alpha$ for all $t$ (otherwise there is a subsequence with this property and we can apply the following argument to this subsequence). Define the strategy $\hat{p} \in \Delta^{M}$ by $\hat{p}_{i}=1 / s^{\prime}$ for $i=1, \ldots, s^{\prime}$ and $\hat{p}_{i}=0$ otherwise. We will show that $\hat{p} q^{t} \sigma$ is t-dominated by $e^{s^{\prime}+1} q^{t} \sigma$ for sufficiently large $t$, which contradicts Lemma 4.10 and therefore completes the proof. So we are left to show that for $t$ sufficiently large

$$
F_{e^{u^{\prime}+1} q^{t} \sigma}^{t}(k) \leq F_{\hat{p} q^{t} \sigma}^{t}(k) \text { for every } k=1, \ldots, m n .
$$

(Since the probability distributions $e^{s^{\prime}+1} q^{t} \sigma$ and $\hat{p} q^{t} \sigma$ are clearly different, at least one of the inequalities in (4.11) must be strict.)

Let $k \in\{1, \ldots, m n\}$. By (b) and Lemma 4.3 we can choose $t_{1}$ such that for all $t \geq t_{1}$ we have

$$
a_{\sigma(j, j), k}^{t} \geq s a_{\sigma\left(s^{\prime}+1, j\right), k}^{t} \text { for all } j=1, \ldots, s^{\prime}-1 .
$$

Also by (b) and Lemma 4.3 we can choose $t_{2}$ such that for all $t \geq t_{2}$ we have

$$
\alpha a_{\sigma\left(s^{\prime}, s^{\prime}\right), k}^{t} \geq 2 s q_{s^{\prime}}^{t} a_{\sigma\left(s^{\prime}+1, s^{\prime}\right), k^{\prime}}^{t}
$$

By (b), (c), and Lemma 4.3 we can choose $t_{3}$ such that for all $t \geq t_{3}$ we have

$$
\alpha a_{\sigma\left(s^{\prime}, s^{\prime}\right), k}^{t} \geq 2 s \sum_{j \in J: j \geq s^{\prime}+1} q_{j}^{t} a_{\sigma\left(s^{\prime}+1, j\right), k}^{t}
$$


Then, for $t \geq \max \left\{t_{1}, t_{2}, t_{3}\right\}$, we have

$$
\begin{aligned}
F_{e^{s^{\prime}+1} q^{t} \sigma}^{t}(k)= & \sum_{j \in J: \sigma\left(s^{\prime}+1, j\right) \leq k} q_{j}^{t} a_{\sigma\left(s^{\prime}+1, j\right), k}^{t} \\
= & \sum_{j \in J: j<s^{\prime}, \sigma\left(s^{\prime}+1, j\right) \leq k} q_{j}^{t} a_{\sigma\left(s^{\prime}+1, j\right), k}^{t} \\
& +\sum_{j \in J: j \geq s^{\prime}, \sigma\left(s^{\prime}+1, j\right) \leq k} q_{j}^{t} a_{\sigma\left(s^{\prime}+1, j\right), k}^{t} \\
\leq & \sum_{j \in J: j<s^{\prime}} \frac{1}{s} q_{j}^{t} a_{\sigma(j, j), k}^{t}+\frac{1}{2 s} \alpha a_{\sigma\left(s^{\prime}, s^{\prime}\right), k}^{t}+\frac{1}{2 s} \alpha a_{\sigma\left(s^{\prime}, s^{\prime}\right), k}^{t} \\
\leq & F_{\tilde{p} q^{\prime} \sigma}^{t}(k),
\end{aligned}
$$

where the first inequality follows from (4.12) -(4.14). This implies (4.11) and completes the proof of the lemma.

Lemma 4.13 has the following converse.

Lemma 4.14 Let $I \subseteq M$ and $J \subseteq N$ satisfy

(i) for every $i \in M$, there is a $j \in J$ such that $\sigma(i, j)<\sigma\left(i^{\prime}, j\right)$ for all $i^{\prime} \in I \backslash\{i\}$;

(ii) for every $j \in N$, there is an $i \in I$ such that $\tau(i, j)<\tau\left(i, j^{\prime}\right)$ for all $j^{\prime} \in J \backslash\{j\}$.

Let $\iota \in \mathbb{N}$. Then there are $p^{t} \in \Delta^{M}$ and $q^{t} \in \Delta^{N}$ with $\operatorname{supp}\left(p^{t}\right)=I$, $\operatorname{supp}\left(q^{t}\right)=J$, and $\left(p^{t}, q^{t}\right) \in E^{t}$.

Proof. Note that, as before, (i) and (ii) imply $|I|=|J|$. If $|I|=|J|=1$, then there is a pure Nash equilibrium $\left(p^{t}, q^{t}\right) \in E^{t}$ with supports $I$ and $J$. Assume now that $|I|=|J| \geq 2$. As in the proof of Lemma 4.13 we may renumber the pure strategies of the players such that

1. a. $I=J=\{1, \ldots, s\}$ for some $s \geq 2$;

b. $\sigma(j, j)<\sigma(i, j)$ for every $j \in J$ and $i \in I \backslash\{j\}$;

c. $\sigma(1,1)<\sigma(2,2)<\ldots<\sigma(s, s)$.

Define $q^{t} \in \Delta^{N}$ with $\operatorname{supp}\left(q^{t}\right)=\{1, \ldots, s\}$ such that

$$
q_{j}^{t} / q_{j-1}^{t}=m n a_{1, m n}^{t} \text { for every } j=2, \ldots, s .
$$

We show that every $p \in \Delta^{M}$ with $\operatorname{supp}(p) \subseteq I$ is a $t$-best reply against $q^{t}$. Since we can define $p^{t}$ analogously and show that every $q \in \Delta^{N}$ with $\operatorname{supp}(q) \subseteq J$ is a $t$-best reply against $p^{t}$, the proof is complete.

So let $p \in \Delta^{M}$ with $\operatorname{supp}(p) \subseteq I$. Assume, contrary to what we wish to prove, that there is a $p^{\prime} \in \Delta^{M}$ such that $p q^{t}$ is $t$-dominated by $p^{\prime} q^{t}$. We first argue 
that without loss of generality $\operatorname{supp}(p) \cap \operatorname{supp}\left(p^{\prime}\right)=\emptyset$. For, suppose that $i$ is an element in this intersection, and let $\alpha:=\min \left\{p_{i}, p_{i}^{\prime}\right\}$. Define $\bar{p}:=1 /(1-$ $\alpha)\left(p-\alpha e^{i}\right)$ and $\bar{p}^{\prime}:=1 /(1-\alpha)\left(p^{\prime}-\alpha e^{i}\right)$. Then $\bar{p}, \bar{p}^{\prime} \in \Delta^{M}$, and $\bar{p} q^{t}$ is still $t$-dominated by $\bar{p}^{\prime} q^{t}$ (since $\bar{p} q^{t}$ and $\bar{p}^{\prime} q^{t}$ arise from $p q^{t}$ and $p^{\prime} q^{t}$, respectively, by first subtracting the same amount from the same coordinates and next rescaling, so that the inequalities of the stochastic clominance relation do not change), whereas $i \notin \operatorname{supp}(\bar{p}) \cap \operatorname{suppp}\left(\bar{p}^{\prime}\right)$ and $\operatorname{supp}(\bar{p}) \subseteq I$.

So assume that $\operatorname{supp}(p) \cap \operatorname{supp}\left(p^{\prime}\right)=\emptyset$, and take $\hat{\imath} \in \operatorname{supp}\left(p^{\prime}\right)$ such that $p_{\hat{\imath}}^{\prime} \geq$ $1 / m$. If $\hat{\imath} \in I$ then let $\hat{\jmath}:=\hat{\imath}$. Then, by condition $(\mathrm{b}), \sigma(\hat{\imath}, \hat{\jmath})<\sigma(i, \hat{\jmath})$ for all $i \in I \backslash\{\hat{\imath}\}$, hence for all $i \in \operatorname{supp}(p)$ in particular. If $\hat{\imath} \in M \backslash I$ then take $\hat{\jmath} \in J$ such that $\sigma(\hat{i}, \hat{\jmath})<\sigma(i, \hat{j})$ for all $i \in I$, hence for all $i \in \operatorname{supp}(p)$ : this is possible by condition (i) in Lemma 4.11. Together with conditions (b) and (c) this implies

$$
\sigma(\hat{\imath}, \hat{\jmath})<\sigma(i, j) \text { for all } i \in \operatorname{supp}(p) \text { and } j \in\{\hat{j}, \ldots, s\} \text {. }
$$

Let $k:=\sigma(\hat{\imath}, \hat{\jmath})$. Then

$$
\begin{aligned}
F_{p q^{t} \sigma}^{t}(k) & =\sum_{l=1}^{k}\left(p q^{t} \sigma\right)_{l} a_{l k}^{t} \\
& =\sum_{j=1}^{j-1} q_{j}^{t}\left(\sum_{i \in I: p_{i}>0, \sigma(i, j)<k} p_{i} a_{\sigma(i, j), k}^{t}\right) \\
& \leq \sum_{j=1}^{\hat{j}-1} q_{j}^{t} a_{1, m n}^{t} \\
& <n q_{\hat{\jmath}-1}^{t} a_{1, m n}^{t} \\
& =q_{\hat{\jmath}}^{t} / m \\
& \leq(1 / m) q_{\hat{\jmath}}^{t} a_{\sigma(\hat{\imath}, \hat{j}), k}^{t} \\
& \leq F_{p^{\prime} q^{t} \sigma}^{t}(k)
\end{aligned}
$$

where the first equality follows from Lemma 4.1; the second equality by (4.16); the first inequality by Lemma 4.2 ; the second (strict) inequality and the third equality by (4.15); the third inequality since $a_{\sigma(\hat{\imath}, \hat{\jmath}), k}^{t} \geq 1$; and the final inequality by Lemma 4.1 and the choice of $p_{\hat{\imath}}^{\prime} \geq 1 / \mathrm{m}$.

Since (4.17) contradicts the assumption that $p q^{t}$ is $t$-dominated by $p^{\prime} q^{t}$, the proof of the lemma is complete.

Lemmata 4.11, 4.13, and 4.14, and Corollary 4.12 can be summarized as follows.

Theorem 4.15 If $I \subseteq M, J \subseteq N$, and if $\left(p^{t}, q^{t}\right)_{t \in \mathbb{N}}$ is a sequence of t-equilibria such that $I=\operatorname{supp}\left(p^{t}\right)$ and $J=\operatorname{supp}\left(q^{t}\right)$ for all $t \in \mathbb{N}$, then (i) and (ii) in Lemma 4.11 hold, $|I|=|J|$, and the sequence of $t$-equilibria converges to the pure strategy combination $(\hat{\imath}, \hat{\jmath})$, where $\hat{\imath}$ and $\hat{\jmath}$ are as in Lemma 4.13. Conversely, if $\emptyset \neq I \subseteq M$ and $\emptyset \neq J \subseteq N$ satisfy (i) and (ii) in Lemma 4.11, then a sequence of t-equilibria with supports $I$ and $J$ for players 1 and 2 , respectively, exists. 
As announced earlier, the results in this section imply that, as $t$ becomes large, the equilibrium behavior of the players converges to max-min play in a specific sense. Take any sequence of $t$-equilibria with (without loss of generality) constant supports $I$ and $J$ of the players' strategies. Then, in the limit, player 1 puts all weight on that strategy (row) in $I$ in which the worst outcome for player 2 with respect to the strategies (columns) in $J$ is maximal among all rows in $I$; and player 2 puts all weight on that column in $J$ in which the worst outcome for player 1 with respect to the rows in $I$ is maximal among all columns in $J$. The next examples illustrate this further.

Example 4.16 Consider the following $3 \times 3$ game, in which the numbers express the ordinal preferences of the players:

$$
\left[\begin{array}{lll}
1,1 & 6,5 & 9,4 \\
5,6 & 2,2 & 8,7 \\
4,9 & 7,8 & 3,3
\end{array}\right] .
$$

In this game, $I:=M=\{1,2,3\}$ and $J:=N=\{1,2,3\}$ clearly satisfy (i) and (ii) in Lemma 4.11. Obviously, $\hat{\imath}=\hat{\jmath}=3$. For every $t \in \mathbb{N}$ the $t$-equilibrium used in the proof of Lemma 4.14 is defined by

$$
p^{t}=q^{t}=\left(\frac{1}{1+A+A^{2}}, \frac{A}{1+A+A^{2}}, \frac{A^{2}}{1+A+A^{2}}\right)
$$

where $A=9(7+t) ! / 8 !(t-1)$ !. In this equilibrium the weights on the first two rows (and columns) converge to 0 and $p_{1}^{t} / p_{2}^{t}$ (and $q_{1}^{t} / q_{2}^{t}$ ) converges to zero as well. That is, the weight on the first row (column) goes to 0 much faster than the weight on the second row (column). The latter phenomenon is not a necessary one: in the present example, for instance, it is also possible to have a sequence of t-equilibria with equal weights on the first two rows (columns). It can be verified that taking $p_{1}^{t}=p_{2}^{t}=q_{1}^{t}=q_{2}^{t}=: \alpha^{t}$ such that $\left(1-2 \alpha^{t}\right) / \alpha^{t}>a_{1,3}^{t}+a_{1,8}^{t}$ for every $t \in \mathbb{I} N$ is again a $t$-equilibrium. Finally, $I=M$ and $J=N$ are the only subsets of pure strategies satisfying (i) and (ii) in Lemma 4.11, hence the only supports of t-equilibria. Hence, in the limit each player plays his third strategy, resulting in the 'payoffs' $(3,3)$.

Example 4.17 Consider the following $3 \times 3$ game:

$$
\left[\begin{array}{lll}
4,4 & 8,5 & 3,6 \\
5,8 & 7,7 & 2,9 \\
6,3 & 9,2 & 1,1
\end{array}\right] .
$$

The following combinations satisfy (i) and (ii) in Lemma 4.11:

(a) $I=\{3\}, J=\{1\}$, resulting in $(3,1)$ in the limit;

(b) $I=\{1\}, J=\{3\}$, resulting in $(1,3)$ in the limit;

(c) $I=\{1,3\}, J=\{2,3\}$, resulting in $(1,2)$ in the limit; 
(d) $I=\{2,3\}, J=\{1,3\}$, resulting in $(2,1)$ in the limit;

(e) $I=M, J=N$, resulting in $(2,2)$ in the limit.

This means that the 'payoff pairs' that can arise as limits of t-equilibria are $(6,3),(3,6),(8,5),(5,8)$, and $(7,7)$.

\subsection{Concluding remarks}

There are some obvious possible extensions of the results in this chapter. First, the definitions and results in Sections 4.2 and 4.4 can be generalized quite easily to games with more than two players. The same, however, is far from obvious for the asymptotic results of Section 4.5. For this reason, we chose to present the entire chapter only for two-person games.

Second, the implicit assumption in the concept of $t$-equilibrium that the value of $t$ is the same for both players can be omitted. All results would also hold for $\left(t_{1}, t_{2}\right)$-equilibria, where $(p, q) \in \Delta^{M} \times \Delta^{N}$ is called a $\left(t_{1}, t_{2}\right)$-equilibrium if $p$ is a $t_{1}$-best reply against $q$ and $q$ is a $t_{2}$-best reply against $p$. This is especially established by the more general results in Chapter 6 .

Third, the degree of stochastic dominance $t$ can be varied continuously instead of in discrete steps. Again, all results would continue to hold for this extension. This is shown in Chapter 6.

Fourth, the assumption of the players having a linear ordering (no indifferences) on the certain alternatives is not an essential one, but it makes the asymptotic results of Section 4.5 much cleaner.

Fifth, modelling incomplete preferences by stochastic dominance is an in our view justifiable but also quite specific choice. Dubra et al. (2004) characterize incomplete preferences satisfying the von Neumann-Morgenstern axioms by identifying characterizing classes of utility functions that play the same role as the classes $\bar{U}^{t}$, cf. the end of Section 4.1. This points at a general approach that can be used to extend some of the results of this chapter. We will study this extension in the Chapter 6. 


\section{Chapter 5}

\section{Stochastic dominance approach to correlated equilibrium}

In a noncooperative game in which the players' preferences are given by von Neumann-Morgenstern utility functions there are two important equilibrium concepts: Nash equilibrium and correlated equilibrium. In a mixed strategy Nash equilibrium each player plays optimally - maximizes expected utility against the conjectured strategy combination of his opponents. A correlated equilibrium, on the other hand, is a probability distribution over pure outcomes of the game, not necessarily arising from a mixed strategy profile, such that for each player it is optimal to follow the "advice" resulting from a draw from this distribution. For a correlated equilibrium to make sense some communication mechanism between the players is presumed.

In this chapter we assume that the players have complete preferences over pure outcomes and incomplete preferences over lotteries over pure outomes, arising from playing mixed strategies. Hence, we drop the completeness assumption, which can be regarded as a strong assumption, from the von NeumannMorgenstern conditions. For the consequences of considering incomplete preferences we refer to [Dubra et al, 2004]. Our approach fits into the general setting of that paper.

More specifically, we consider two-person noncooperative games in which players have incomplete preferences over lotteries and evaluate these lotteries by a stochastic dominance criterion, namely the criterion arising from $t$-th degree stochastic dominance. Here $t$ is a natural number that indicates the players' aversion to "bad" outcomes. The preference relation over mixed strategies induced by $t$-th order stochastic dominance is incomplete. The degree of completeness of the preference relation increases with $t$; in the limit for $t$ approaching infinity, the preference relation becomes a complete lexicographic ordering (see 
Chapter 4). In terms of stochastic dominance a mixed strategy of a player is a best reply if it induces a stochastically undominated probability distribution over the outcomes. A pair of best replies is called an equilibrium or, more specifically referring to $t$-th degree stochastic dominance, a $t$-equilibrium.

In Chapter 4 the relation betwcen Nash equilibrium and $t$-equilibrium is investigated. It is shown that the set of $t$-equilbria can be characterized as the union of Nash equilibria over a certain class (depending on $t$ ) of utility functions that represent the given preference relation over pure outcomes. This result generalises the result in [Fishburn, 1980] for first degree stochastic dominance. It is shown that an increasing value of $t$ can be interpreted as an increasing aversion towards "bad" outcomes, since the corresponding classes of utility functions contain increasingly concave functions as $t$ becomes larger. Furthermore, possible supports and resulting strategy profiles for series of $t$-equilibria and their limits are considered.

In this chapter we introduce the concept of correlated $t$-equilibrium, and we perform an analogous investigation of the relation between correlated $t$-equilibria and correlated equilibria. We show that if a probability distribution is a correlated equilibrium with respect to some utility representation it is also a correlated $t$-equilibrium. This result together with the existence of correlated equilibria implies existence of correlated $t$-equilibria. We study sequences of correlated $t$-equilibria and their limits as $t$ tends to infinity. We show that given the support of the limit of a sequence of correlated $t$-equilibria we can, for any probability distribution over this support, construct a sequence of correlated $t$-equilibria that converges to this probability distribution over the support.

The outline of this chapter is as follows. We introduce correlated t-equilibrium, the main concept we study in the remainder of this chapter. We proceed by showing in section 5.2 that the set of correlated $t$-equilibria is always nonempty and investigate the relationship between correlated $t$-equilibrium and correlated equilibrium. In the last section, section 5.3, we study sequences of correlated $t$-equilibria as $t$ tends to infinity.

\subsection{Correlated equilibrium and correlated $t$-equilibria}

We define the concepts of correlated equilibrium, following [Aumann, 1974], and correlated $t$-equilibrium. Let $\mu \in \Delta(M \times N)$ be a probability clistribution over the pure strategy pairs. We clefine the sets

$$
\begin{array}{ll}
\operatorname{supp}_{1}(\mu):= & \{i \in M \mid \exists j \in N \text { such that } \mu(i, j)>0\} \text { and } \\
\operatorname{supp}_{2}(\mu):= & \{j \in N \mid \exists i \in M \text { such that } \mu(i, j)>0\} .
\end{array}
$$

In the concept of correlated equilibrium for every player every pure strategy in this player's support must be a best reply to the conditional probability distribution over his opponent's strategies that is induced by this pure strategy. Hence, for player 1 , every row $i \in \operatorname{supp}_{1}(\mu)$ must be a best reply to the probability 
distribution $(\mu \mid i) \in \Delta^{N}$ that is defined by

$$
(\mu \mid i)(j):=\frac{\mu(i, j)}{\sum_{j^{\prime} \in N} \mu\left(i, j^{\prime}\right)} .
$$

A similar relation holds for player 2 . We now give the corresponding definition if strategies are evaluated in terms of stochastic dominance.

Definition 5.1 Let $t \in \mathbb{N}$ be given. The probability distribution $\mu^{t} \in \Delta(M \times N)$ is called a correlated t-equilibrium if

(1) for every $i \in \operatorname{supp}_{1}\left(\mu^{t}\right)$ the pure strategy $i$ is a t-best reply to $\left(\mu^{t} \mid i\right)$ and

(2) for every $j \in$ supp $_{2}\left(\mu^{t}\right)$ the pure strategy $j$ is a t-best reply to $\left(\mu^{t} \mid j\right)$.

\subsection{Existence of correlated $t$-equilibria}

In Chapter 4 a characterization of the set of $t$-best replies in terms of expected utility with respect to the class $U^{t}$ of utility functions is given by the following lemma.

Lemma 5.2 Let $p \in \Delta^{M} . q \in \Delta^{N}$, and $t \geq 1$. Then $p$ is a $t$-best reply against $q$ if and only if there is a $u^{l} \in U^{t}$ such that

$$
\sum_{i=1}^{m} \sum_{j=1}^{n} p_{i} q_{j} u_{\sigma(i, j)}^{t} \geq \sum_{i=1}^{m} \sum_{j=1}^{n} p_{i}^{\prime} q_{j} u_{\sigma(i, j)}^{t}
$$

for all $p^{\prime} \in \Delta^{M I}$.

The following lemma states that every correlated equilibrium with respect to utility functions in $U^{t}$ is a correlated $t$-equilibrium.

Lemma 5.3 Let $\mu \in \Delta(M \times N)$ be a correlated equilibrium with respect to some pair $\left(u_{1}, u_{2}\right) \in U_{\sigma}^{t} \times U_{\tau}^{t}$. Then $\mu$ is a correlated $t$-equilibrium.

Proof. By Lemma 5.2 a strategy $p$ of player 1 is a $t$-best reply to a strategy $q$ of player 2 if and only if there exists a utility function $u^{t} \in U_{\sigma}^{t}$ such that $p$ is a best reply to $q$ with respect to $u^{t}$. Let $\mu$ be a correlated equilibrium for some utility representation $\left(u_{1}^{t}, u_{2}^{t}\right) \in U_{\sigma}^{t} \times U_{\tau}^{t}$. For player 1 this implies that for all $i \in \operatorname{supp}_{1}(\mu)$ this $i$ is a $t$-best reply to $(\mu \mid i)$. The same argument holds for player 2 and hence, $\mu$ is a correlated $t$-equilibrium.

Since the set of correlated equilibria is always nonempty, we have the following existence result. Another way of getting to this result is by noticing that every $t$-equilibrium (which always exists) is also a correlated $t$-equilibrium.

Corollary 5.4 Correlated $t$-equilibria exist for all $t \geq 1$.

The following example shows that the converse of Lemma 5.3 is not true: there exist correlated $t$-equilibria that are not a correlated equilibrium for any choice of utility functions. 
Example 5.5 Consider the game

$$
\left(\begin{array}{ll}
4,2 & 1,1 \\
3,3 & 2,4
\end{array}\right)
$$

where the numbers represent the ordinal preferences $\sigma$ and $\tau$. We construct correlated t-equilibria of the form

$$
\mu^{t}:=\left(\begin{array}{ll}
\mu_{11}^{t} & \mu_{12}^{t} \\
\mu_{21}^{t} & \mu_{22}^{t}
\end{array}\right)
$$

where all coefficients are strictly positive. For player 1 the first row is a t-best reply to $q_{1}:=\left(\mu^{t} \mid(\right.$ row 1$\left.)\right)$ if and only if there exists a $k_{1} \in\{1,2,3,4\}$ such that

$$
F_{e_{1} q_{1}}^{t}\left(k_{1}\right)<F_{e_{2} q_{1}}^{t}\left(k_{1}\right)
$$
Therefore, from $F_{r}^{t}=r A^{t}$ for row 1 with $r=\left(\frac{\mu_{12}^{t}}{\mu_{11}^{t}+\mu_{12}^{t}}, 0,0, \frac{\mu_{11}^{t}}{\mu_{11}^{t}+\mu_{12}^{t}}\right)$ we com-
pute

$$
\begin{array}{lc}
F_{e_{1} q_{1}}^{t}(1)= & \frac{\mu_{12}^{t}}{\mu_{11}^{t}+\mu_{12}^{t}} \\
F_{e_{1} q_{1}}^{t}(2)= & \frac{t \mu_{12}^{t}}{\mu_{11}^{t}+\mu_{12}^{t}} \\
F_{e_{1} q_{1}}^{t}(3)= & \frac{t(t+1) \mu_{12}^{t}}{2\left(\mu_{11}^{t}+\mu_{12}^{t}\right)} \\
F_{e_{1} q_{1}}^{t}(4)= & \frac{t(t+1)(t+2) \mu_{12}^{t}+6 \mu_{11}^{t}}{6\left(\mu_{11}^{t}+\mu_{12}^{t}\right)}
\end{array}
$$

and for row 2 with $r=\left(0, \frac{\mu_{12}^{t}}{\mu_{11}^{t}+\mu_{12}^{t}}, \frac{\mu_{11}^{t}}{\mu_{11}^{t}+\mu_{12}^{t}}, 0\right)$ we obtain

$$
\begin{array}{lc}
F_{e_{2} q_{1}}^{t}(1)= & 0 \\
F_{e_{2} q_{1}}^{t}(2)= & \frac{\mu_{12}^{t}}{\mu_{11}^{t}+\mu_{12}^{t}} \\
F_{e_{2} q_{1}}^{t}(3)= & \frac{t \cdot \mu_{12}^{t}+\mu_{11}}{\mu_{11}^{t}+\mu_{12}^{t}} \\
F_{e_{2} q_{1}}^{t}(4)= & \frac{t(t+1) \mu_{12}^{t}+2 t \cdot \mu_{11}^{t}}{2\left(\mu_{11}^{t}+\mu_{12}^{t}\right)}
\end{array}
$$

From this we see that row 1 is a t-best reply against $\left(\mu^{t} \mid\right.$ row 1$)$ if and only if either $F_{e_{1} q_{1}}^{t}(3)<F_{e_{2} q_{1}}^{t}(3)$ or $F_{e_{1} q_{1}}^{t}(4)<F_{e_{2} q_{1}}^{t}(4)$. Solving the inequalities
yields for $t \neq 1$

$$
\mu_{12}^{t}<\frac{2 \mu_{11}^{t}}{t(t-1)} \text { or } \mu_{12}^{t}<\frac{6 \mu_{11}^{t}}{t(t+1)}
$$

Notice that the second row is automatically a t-best reply to $q_{2}:=\left(\mu^{t} \mid(\right.$ row 2$\left.)\right)$, since $F_{e_{2} q_{2}}^{t}(1)=0$ and $F_{e_{1} q_{2}}^{t}(1) \neq 0$. If we do the same for player 2 and solve the inequalities and combine them with (5.1), we find that $\mu^{t}$ is a correlated t-equilibrium if and only if

$$
\mu_{12}^{t}<\frac{6 \cdot \min \left\{\mu_{11}^{t}, \mu_{22}^{t}\right\}}{t(t+1)}
$$


On the other hand we investigate when $\mu^{t}$ is a correlated equilibrium with respect to some pair of utility functions $u^{t}=\left(u_{1}^{t}, u_{2}^{t}\right)$. Therefore, we write the game as

$$
\left(\begin{array}{ll}
u_{14}^{t}, u_{22}^{t} & u_{11}^{t}, u_{21}^{t} \\
u_{13}^{t}, u_{23}^{t} & u_{12}^{t}, u_{24}^{t}
\end{array}\right)
$$

where the first index refers to the player and the second to the ranking in $\sigma$ and $\tau$ respectively. Now, $\mu^{t}$ is a correlated equilibrium with respect to $u^{t}$ if and only if for player 1

This implies

$$
\begin{aligned}
& \mu_{11}^{t} u_{14}^{t}+\mu_{12}^{t} u_{11}^{t} \geq \mu_{11}^{t} u_{13}^{t}+\mu_{12}^{t} u_{12}^{t} \text { and } \\
& \mu_{21}^{t} u_{13}^{t}+\mu_{22}^{t} u_{12}^{t} \geq \mu_{21}^{t} u_{14}^{t}+\mu_{22}^{t} u_{11}^{t} .
\end{aligned}
$$

For player 2 the conditions are

$$
\frac{\mu_{21}^{t}}{\mu_{22}^{t}} \leq \frac{u_{12}^{t}-u_{11}^{t}}{u_{14}^{t}-u_{13}^{t}} \leq \frac{\mu_{11}^{t}}{\mu_{12}^{t}}
$$

$$
\begin{aligned}
& \mu_{11}^{t} u_{22}^{t}+\mu_{21}^{t} u_{23}^{t} \geq \mu_{11}^{t} u_{21}^{t}+\mu_{21}^{t} u_{24}^{t} \text { and } \\
& \mu_{12}^{t} u_{21}^{t}+\mu_{22}^{t} u_{24}^{t} \geq \mu_{12}^{t} u_{22}^{t}+\mu_{22}^{t} u_{23}^{t} .
\end{aligned}
$$

This implies

$$
\frac{\mu_{12}^{t}}{\mu_{22}^{t}} \leq \frac{u_{24}^{t}-u_{23}^{t}}{u_{22}^{t}-u_{21}^{t}} \leq \frac{\mu_{11}^{t}}{\mu_{21}^{t}}
$$

Combining the relations for player 1 and 2 yields that if $\mu^{t}$ is a correlated equilibrium with respect to $u^{t}$ then

$$
\frac{\mu_{21}^{t}}{\mu_{22}^{t}} \leq \frac{\mu_{11}^{t}}{\mu_{12}^{t}}
$$

Now consider

$$
\mu^{t}:=\frac{1}{N(t)}\left(\begin{array}{cc}
t(t-1) & 1 \\
2 t^{2}(t-1)^{2} & t(t-1)
\end{array}\right)
$$

with normalization factor

$$
N(t)=2 t(t-1)(1+t(t-1))+1
$$

Notice that

implying

$$
\frac{\mu_{21}^{t}}{\mu_{22}^{t}}=2 t(t-1) \text { and } \frac{\mu_{11}^{t}}{\mu_{12}^{t}}=t(t-1)
$$

$$
\frac{\mu_{21}^{t}}{\mu_{22}^{t}}>\frac{\mu_{11}^{t}}{\mu_{12}^{t}}
$$

Hence, it follows from (5.3) that there exists no pair of utility functions $u^{t}$ such that $\mu^{t}$ is a correlated equilbrium with respect to $u^{t}$. On the other hand, for all $t \mu_{11}^{t}=\mu_{22}^{t}=\min \left\{\mu_{11}^{t}, \mu_{22}^{t}\right\}$ and

$$
1<\frac{6 t(t-1)}{t(t+1)}
$$

for all $t>1$. With (5.2) $\mu^{t}$ is a correlated $t$-equilibrium for all $t>1$. 


\subsection{Sequences of correlated $t$-equilibria}

Next we consider limits of sequences of correlated t-equilibria,

$$
\mu:=\lim _{t \rightarrow \infty} \mu^{t}
$$

The theorem in this section states that, given the support of the limit of a sequence of correlated $t$-equilibria, for any probability distribution over this support there exists another sequence of correlated $t$-equilibria that converges to this support. First we show by an example that the limit of a sequence of correlated $t$-equilibria need not be a pure strategy, as was the case with t-equilibria (sec chapter 4 ).

Example 5.6 Consider again the following game with ordinal preferences:

$$
\left(\begin{array}{ll}
4,2 & 1,1 \\
3,3 & 2,4
\end{array}\right) \text {. }
$$

In example 5.5 we showed that

$$
\mu^{t}:=\left(\begin{array}{ll}
\mu_{11}^{t} & \mu_{12}^{t} \\
\mu_{21}^{t} & \mu_{22}^{t}
\end{array}\right)
$$

is a correlated t-equilibrium if and only if

$$
\mu_{12}^{t}<\frac{6 \cdot \min \left\{\mu_{11}^{t}, \mu_{22}^{t}\right\}}{t(t+1)}
$$

which implies

$$
\lim _{t \rightarrow \infty} \mu_{12}^{t}=0
$$

On the other hand, there are no restrictions on the other elements $\mu_{11}^{t}, \mu_{21}^{t}$ and $\mu_{22}^{t}$, which implies that the limit can be

$$
\mu=\left(\begin{array}{cc}
\mu_{11} & 0 \\
\mu_{21} & \mu_{22}
\end{array}\right)
$$

for any $\mu_{11}, \mu_{21}, \mu_{22}$ satisfying $\mu_{11}+\mu_{21}+\mu_{22}=1$. Thus for any choice of these coefficients there is a sequence of correlated t-equilibria that converges to this choice.

Theorem 5.7 Let $\left(\mu^{t}\right)_{t=1}^{\infty}$ be a sequence of correlated t-equilibria and let $\mu$ be the limit of this sequence. Furthermore, let $\sup p(\mu)$ be the set of pairs $(i, j) \in$ $M \times N$ that have positive weight in $\mu$. Then, for cvery probability distribution $\bar{\mu}$ such that $\operatorname{supp}(\bar{\mu}) \subseteq \operatorname{supp}(\mu)$ there is a sequence $\left(\bar{\mu}^{t}\right)_{t=1}^{\infty}$ of correlated t-equilibria that converges to $\bar{\mu}$. 
Proof. (1) We first look at the case in which $\bar{\mu}$ is such that $\operatorname{supp}(\bar{\mu})=$ $\operatorname{supp}(\mu)$. This part of the proof is structured as follows. We fix a pair $\left(i^{*}, j^{*}\right) \in$ $\operatorname{supp}(\mu)$. We then define $\tilde{\mu}$ by

$$
\tilde{\mu}(i, j):=\left\{\begin{array}{cc}
\alpha \cdot \mu(i, j)=\bar{\mu}(i, j) & \text { if }(i, j)=\left(i^{*}, j^{*}\right) \\
\mu(i, j) & \text { else, }
\end{array}\right.
$$

and let $\alpha:=\frac{\bar{\mu}(i, j)}{\mu(i, j)}$. We construct $\tilde{\mu}^{t}$ such that $\tilde{\mu}=\lim _{t \rightarrow \infty} \tilde{\mu}^{t}$. Before we can construct the sequence $\tilde{\mu}^{t}$ we introduce some notation and properties. The relevant definitions and results are formulated for player 1; the statements for player 2 are analogous. Repeating this construction for all pairs $\left(i^{*}, j^{*}\right)$ such that $\mu\left(i^{*}, j^{*}\right) \neq \bar{\mu}\left(i^{*}, j^{*}\right)$ leads to the sequence $\bar{\mu}^{t}$ which converges to $\bar{\mu}$.

Let $i \in M$. Then we define

$$
q_{i}^{t}:=\left(\mu^{t} \mid i\right)
$$

We assume w.l.o.g. that the support of $q_{i}^{t}$ is constant, since otherwise we can always take a subsequence that has this property. Therefore, we write

$$
J_{i}:=\operatorname{supp}\left(q_{i}^{t}\right) .
$$

We will use an altomative notation for the matrix elements $a_{k l}^{t}$ of the matrix $A^{t}$, because they only depend on $(l-k)$

$$
b^{t}(l-k):=a_{k l}^{t} \text {. }
$$

Notice that the factors $b^{t}(l-k)$ are polynomials in $t$ of degree $l-k$. By the assumption that $\mu$ is the limit of a sequence of correlated $t$-equilibria, $i$ is a t-best reply to $q_{i}^{t}$ for all $t$. Hence, for all $i^{\prime} \neq i \in M$ there exists a $k_{i i^{\prime}} \in\{1, \ldots, m n\}$ such that

$$
F_{e_{2} q_{i}^{t}}^{t}\left(x_{k_{i i^{\prime}}}\right)<F_{e_{i^{\prime}} q_{i}^{i}}^{t}\left(x_{k_{i i^{\prime}}}\right) .
$$

Since the set $\{1, \ldots, m n\}$ is finite, we assume w.l.o.g. that $k_{i i^{\prime}}$ does not depend on $t$. By definition

$$
\begin{aligned}
F_{e_{i} q_{i}^{t}}^{t}\left(x_{k_{i i^{\prime}}}\right) & =\sum_{j \in J_{2}} b^{t}\left(k_{i i^{\prime}}-\sigma(i, j)\right) \cdot q_{i}^{t}(j), \\
F_{e_{i^{\prime}} q_{i}^{t}}^{t}\left(x_{k_{i i^{\prime}}}\right) & =\sum_{j \in J_{i}} b^{t}\left(k_{i i^{\prime}}-\sigma\left(i^{\prime}, j\right)\right) \cdot q_{i}^{t}(j) .
\end{aligned}
$$

Combining the last three relations yields for all $t$

$$
C_{i i^{\prime}}\left(\mu^{t}\right):=\frac{\sum_{j \in J_{2}} b^{t}\left(k_{i i^{\prime}}-\sigma(i, j)\right) \cdot q_{i}^{t}(j)}{\sum_{j \in J_{i}} b^{t}\left(k_{i i^{\prime}}-\sigma\left(i^{\prime}, j\right)\right) \cdot q_{i}^{t}(j)}<1 .
$$

Multiplying enumerator and denominator by $\sum_{j^{\prime} \in N} \mu^{t}\left(i, j^{\prime}\right)$ yields

$$
C_{i i^{\prime}}\left(\mu^{t}\right)=\frac{\sum_{j \in J_{i}} b^{t}\left(k_{i i^{\prime}}-\sigma(i, j)\right) \cdot \mu^{t}(i, j)}{\sum_{j \in J_{i}} b^{t}\left(k_{i i^{\prime}}-\sigma\left(i^{\prime}, j\right)\right) \cdot \mu^{t}(i, j)}<1 .
$$


We investigate $\lim _{t \rightarrow \infty} C_{i i^{\prime}}\left(\mu^{t}\right)$, and therefore consider the "leading terms" in both enumerator and denominator. To specify this, we define the following nonempty sets:

$$
\begin{aligned}
& J_{i i^{\prime}}^{e}\left(\mu^{t}\right):=\left\{j \in J_{i} \mid \lim _{t \rightarrow \infty} \frac{b^{t}\left(k_{i i^{\prime}}-\sigma(i, j)\right) \cdot \mu^{t}(i, j)}{\sum_{j \in J_{i}} b^{t}\left(k_{i i^{\prime}}-\sigma(i, j)\right) \cdot \mu^{t}(i, j)}>0\right\}, \\
& J_{i i^{\prime}}^{d}\left(\mu^{t}\right):=\left\{j \in J_{i} \mid \lim _{t \rightarrow \infty} \frac{b^{t}\left(k_{i i^{\prime}}-\sigma\left(i^{\prime}, j\right)\right) \cdot \mu^{t}(i, j)}{\sum_{j \in J_{i}} b^{t}\left(k_{i i^{\prime}}-\sigma\left(i^{\prime}, j\right)\right) \cdot \mu^{t}(i, j)}>0\right\},
\end{aligned}
$$

and obtain $C_{i i^{\prime}}\left(\mu^{t}\right)=$

$$
\frac{\sum_{j \in J_{i^{\prime} i^{\prime}}\left(\mu^{t}\right)} b^{t}\left(k_{i i^{\prime}}-\sigma(i, j)\right) \cdot \mu^{t}(i, j)+\sum_{j \in J_{i} \backslash J_{i i^{\prime}}\left(\mu^{t}\right)} b^{t}\left(k_{i i^{\prime}}-\sigma(i, j)\right) \cdot \mu^{t}(i, j)}{\sum_{j \in J_{i^{\prime}}^{d}\left(\mu^{t}\right)} b^{t}\left(k_{i i^{\prime}}-\sigma\left(i^{\prime}, j\right)\right) \cdot \mu^{t}(i, j)+\sum_{j \in J_{i} \backslash J_{i^{\prime}}^{d}\left(\mu^{t}\right)} b^{t}\left(k_{i i^{\prime}}-\sigma\left(i^{\prime}, j\right)\right) \cdot \mu^{t}(i, j)}
$$

and hence,

$$
\lim _{t \rightarrow \infty} C_{i i^{\prime}}\left(\mu^{t}\right)=\lim _{t \rightarrow \infty} \frac{\sum_{j \in J_{i i^{\prime}}^{e}\left(\mu^{t}\right)} b^{t}\left(k_{i i^{\prime}}-\sigma(i, j)\right) \cdot \mu^{l}(i, j)}{\sum_{j \in J_{i i^{\prime}}^{d}\left(\mu^{t}\right)} b^{t}\left(k_{i i^{\prime}}-\sigma\left(i^{\prime}, j\right)\right) \cdot \mu^{t}(i, j)} .
$$

Next we define the following partition of $J_{i}$

$$
\begin{aligned}
& P_{i}^{1}:=\left\{j \in J_{i} \mid \mu(i, j)>0\right\} ; \\
& P_{i}^{2}:=\left\{j \in J_{i} \mid \mu(i, j)=0\right\} .
\end{aligned}
$$
that

Since $\lim _{t \rightarrow \infty} \mu^{t}(i, j)=0$ for all $j \in P_{i}^{2}$ there exists for all $t$ a $t^{\prime}(t)>t$ such

$$
\mu^{t^{\prime}(t)}(i, j)<\frac{\mu^{t}(i, j)}{t^{m n}}
$$

for all $j \in P_{i}^{2}$ and all $i$. Then $\mu^{t^{\prime}(t)}$ is a correlated $t$-equilibrium by Lemma 4.4 since $t^{\prime}(t)>t$ and $\mu^{t^{\prime}(t)}$ is a correlated $t^{\prime}(t)$-equilibrium.

We prove that

$$
\tilde{\mu}^{t}(i, j):=\left\{\begin{array}{cc}
\alpha \cdot \mu^{t^{\prime}(t)}(i, j) & \text { if }(i, j)=\left(i^{*}, j^{*}\right) \\
\mu^{t^{(t)}}(i, j) & \text { else }
\end{array}\right.
$$

is a sequence with the property that for all $i \in M$ it holds that $C_{i i^{\prime}}\left(\tilde{\mu}^{t}\right)$ for all $i^{\prime} \neq i$ that converges to $\tilde{\mu}$. Notice that $\tilde{\mu}^{t}$ is not a probability distribution (the sum of the elements is unequal to one), but if we repeat this procedure Obviously, $\tilde{\mu}^{t}$ converges to $\tilde{\mu}$, so it remains to show that $\tilde{\mu}^{t}$ is a correlated $t$ equilibrium for all $t$. We prove this using the following auxilary result. This result is only formulated for player 1 ; the proof of the analogous statement for player 2 is similar. 
Claim 5.8 Let $\left(\mu^{t}\right)_{t=1}^{\infty}$ be a sequence of correlated t-equilibria such that $\mu=$ $\lim _{t \rightarrow \infty} \mu^{t}$. Then the sequence $\left(\mu^{t^{\prime}(t)}\right)_{t=1}^{\infty}$, where $t^{\prime}(t)$ is as defined in (5.5) satisfies
(A) $\lim _{t \rightarrow \infty} \mu^{t^{\prime}(t)}=\mu$;
(B) $\left|J_{i i^{\prime}}^{e}\left(\mu^{t^{\prime}(t)}\right)\right|=\left|J_{i i^{\prime}}^{d}\left(\mu^{t^{\prime}(t)}\right)\right|=1$ for all $i, i^{\prime} \in M$;
(C) $\lim _{t \rightarrow \infty} C_{i i^{\prime}}\left(\mu^{t^{\prime}(t)}\right)=0$ for all $i, i^{\prime} \in M$.

Proof. (A) This is immediately clear, since $\mu^{t^{\prime}(t)}$ is a subsequence of $\mu^{t}$.

(B) Take some $i, i^{\prime} \in M$. Then (5.4) yields

$$
\begin{aligned}
& \sum_{j \in J_{i}} b^{t}\left(k_{i i^{\prime}}-\sigma(i, j)\right) \cdot \mu^{t^{\prime}(t)}(i, j) \\
= & \sum_{j \in P_{i}^{1}} b^{t}\left(k_{i i^{\prime}}-\sigma(i, j)\right) \cdot \mu^{t^{\prime}(t)}(i, j)+\sum_{j \in P_{i}^{2}} b^{t}\left(k_{i i^{\prime}}-\sigma(i, j)\right) \cdot \mu^{t^{\prime}(t)}(i, j) \\
< & \sum_{j \in P_{i}^{\mathrm{l}}} b^{t}\left(k_{i i^{\prime}}-\sigma(i, j)\right) \cdot \mu^{t^{\prime}(t)}(i, j)+\sum_{j \in P_{t}^{2}} b^{t}\left(k_{i i^{\prime}}-\sigma(i, j)\right) \cdot \frac{\mu^{t}(i, j)}{t^{m n}} .
\end{aligned}
$$

Since $\left(k_{i i^{\prime}}-\sigma(i, j)\right)<m n-1$ for all $i, i^{\prime}$ we have

$$
\lim _{t \rightarrow \infty} \frac{b^{t}\left(k_{i i^{\prime}}-\sigma(i, j)\right)}{t^{m n}}=0
$$

and hence,

$$
\lim _{t \rightarrow \infty} \sum_{j \in P_{i}^{2}} b^{t}\left(k_{i i^{\prime}}-\sigma(i, j)\right) \cdot \frac{\mu^{t}(i, j)}{t^{m n}}=0
$$

implying $J_{i i^{\prime}}^{e}\left(\mu^{l}\right) \subseteq P_{i}^{1}$ and

$$
\begin{array}{r}
\lim _{t \rightarrow \infty} \frac{\sum_{j \in J_{i i^{\prime}}^{e}\left(\mu^{t^{\prime}(t)}\right)} b^{t}\left(k_{i i^{\prime}}-\sigma(i, j)\right) \cdot \mu^{t^{\prime}(t)}(i, j)}{\sum_{j \in J_{i i^{\prime}}^{d}\left(\mu^{t^{\prime}(t)}\right)} b^{t}\left(k_{i i^{\prime}}-\sigma\left(i^{\prime}, j\right)\right) \cdot \mu^{t^{\prime}(t)}(i, j)} \\
=\lim _{t \rightarrow \infty} \frac{\sum_{j \in P_{i}^{1}} b^{t}\left(k_{i i^{\prime}}-\sigma(i, j)\right) \cdot \mu(i, j)}{\sum_{j \in J_{i i^{\prime}}^{d}\left(\mu^{t^{\prime}(t)}\right)} b^{t}\left(k_{i i^{\prime}}-\sigma\left(i^{\prime}, j\right)\right) \cdot \mu^{t^{\prime}(t)}(i, j)} .
\end{array}
$$


Furthermore, all the $b^{t}\left(k_{i i^{\prime}}-\sigma(i, j)\right)$ have different degree and therefore there exists a $\hat{j} \in P_{i}^{1}$ such that

$$
\begin{gathered}
\lim _{t \rightarrow \infty} \frac{\sum_{j \in J_{i^{\prime}}^{\prime}\left(\mu^{\prime}\right)} b^{t}\left(k_{i i^{\prime}}-\sigma(i, j)\right) \cdot \mu(i, j)}{\sum_{j \in J_{i^{\prime}}^{\prime \prime}\left(\mu^{\prime}(t)\right)} b^{t}\left(k_{i i^{\prime}}-\sigma\left(i^{\prime}, j\right)\right) \cdot \mu^{t^{\prime}(t)}(i, j)} \\
=\lim _{t \rightarrow \infty} \frac{b^{t}\left(k_{i i^{\prime}}-\sigma(i, j)\right) \cdot \mu(i, \hat{\jmath})}{\sum_{j \in J_{i^{\prime}}^{\prime \prime}\left(\mu^{t^{\prime}(t)}\right)} b^{t}\left(k_{i i^{\prime}}-\sigma\left(i^{\prime}, j\right)\right) \cdot \mu^{t^{\prime}(t)}(i, j)}
\end{gathered}
$$

and hence, $\left|J_{i i^{\prime}}^{e}\left(\mu^{t}\right)\right|=1$. Exactly the same argument holds for the denominator. Thus, there exists a $\bar{j} \in P_{i}^{1}$ such that

$$
\lim _{t \rightarrow \infty} \frac{b^{t}\left(k_{i i^{\prime}}-\sigma(i, \hat{\jmath})\right) \cdot \mu(i, \hat{\jmath})}{\sum_{j \in J_{t^{\prime},}^{\prime \prime}\left(\mu^{\prime}\right)} b^{t}\left(k_{i i^{\prime}}-\sigma\left(i^{\prime}, j\right)\right) \cdot \mu^{t}(i, j)}=\lim _{t \rightarrow \infty} \frac{b^{t}\left(k_{i i^{\prime}}-\sigma(i, \hat{\jmath})\right) \cdot \mu(i, \hat{\jmath})}{b^{t}\left(k_{i i^{\prime}}-\sigma\left(i^{\prime}, \bar{j}\right)\right) \cdot \mu(i, \bar{j})}
$$

and $\left|J_{i i^{\prime}}^{d}\left(\mu^{\ell}\right)\right|=1$.

(C) Take some $i, i^{\prime} \in M I$. Then from the proof of (B) and (5.4) it follows that there exist $\hat{j}, \bar{j} \in P_{i}^{1}$ such that

$$
\lim _{t \rightarrow \infty} C_{i i^{\prime}}\left(\mu^{t}\right)=\lim _{t \rightarrow \infty} \frac{b^{t}\left(k_{i i^{\prime}}-\sigma(i, \hat{\jmath})\right) \cdot \mu(i, \hat{\jmath})}{b^{t}\left(k_{i i^{\prime}}-\sigma\left(i^{\prime}, \bar{j}\right)\right) \cdot \mu(i, \bar{j})}
$$

Since $\sigma$ admits no indifference, $b^{t}\left(k_{i i^{\prime}}-\sigma(i, \hat{\jmath})\right)$ and $b^{t}\left(k_{i i^{\prime}}-\sigma\left(i^{\prime}, \bar{j}\right)\right)$ have different degree and

$$
\lim _{t \rightarrow \infty} C_{i i^{\prime}}\left(\mu^{t}\right) \in\{0, \infty\} \text {. }
$$

On the other hand $C_{i i^{\prime}}\left(\mu^{t}\right)<1$ for all $t$, since $\mu^{t}$ is a correlated $t$-equilibrium for all $t$. Therefore,

$$
\lim _{t \rightarrow \infty} C_{i i^{\prime}}\left(\mu^{t}\right)=0
$$

which completes the proof of the claim.

As announced at the beginning of the proof, we now define the sequence

$$
\tilde{\mu}^{t}(i, j):=\left\{\begin{array}{cc}
\alpha \cdot \mu^{t^{\prime}(t)}(i, j) & \text { if }(i, j)=\left(i^{*}, j^{*}\right) \\
\mu^{t^{\prime}(t)}(i, j) & \text { else. }
\end{array}\right.
$$

Notice that with (A) of the previous claim it is obvious that $\tilde{\mu}^{t}$ converges to $\tilde{\mu}$. Hence, it remains to show that $\tilde{\mu}^{t}$ is a correlated t-equilibrium for all $t$. We must show that $e_{i^{*}}$ is a $t$-best reply to $\left(\tilde{\mu}^{t} \mid i^{*}\right)$ for all $t$. Take some $i \neq i^{*}$. Then there exist a $k_{i^{*} i} \in\{1, \ldots, m n\}$ and $\hat{\jmath}, \bar{j} \in P_{i^{*}}^{1}$ such that

$$
\begin{aligned}
& \lim _{t \rightarrow \infty} C_{i^{*} i}\left(\mu^{l}\right) \\
= & \lim _{t \rightarrow \infty} \frac{b^{t}\left(k_{i^{*} i}-\sigma\left(i^{*}, \hat{\jmath}\right)\right) \cdot \mu\left(i^{*}, \hat{\jmath}\right)}{b^{t}\left(k_{i^{*} i}-\sigma(i, \bar{j})\right) \cdot \mu\left(i^{*}, \bar{j}\right)}=0 .
\end{aligned}
$$


We distinguish the following cases.

Case $1 . j^{*}=\hat{\jmath}$ and $\hat{\jmath} \neq \hat{j}$. Then

$$
\lim _{t \rightarrow \infty} C_{i^{*}}\left(\tilde{\mu}^{t}\right)=\alpha \cdot \lim _{t \rightarrow \infty} C_{i^{\star} i}\left(\mu^{t}\right)=0,
$$

and hence, $C_{i^{*} i}\left(\tilde{\mu}^{t}\right)<1$ for all $t$ that arc large enough, which implies that for these $t, e_{i^{*}}$ is a $t$-best reply to $\left(\tilde{\mu}^{t} \mid i^{*}\right)$.

Case 2. $j^{*}=\bar{j}$ and $\hat{j} \neq \bar{j}$. Then

$$
\lim _{t \rightarrow \infty} C_{i^{*} i}\left(\tilde{\mu}^{t}\right)=\frac{1}{\alpha} \cdot \lim _{t \rightarrow \infty} C_{i^{*} i}\left(\mu^{t}\right)=0 .
$$

Case 3. $j^{*} \notin\{\bar{j}, \hat{j}\}$ or $j=\hat{j}=\bar{j}$. Then

$$
\lim _{t \rightarrow \infty} C_{i^{+} i}\left(\tilde{\mu}^{t}\right)=\lim _{t \rightarrow \infty} C_{i^{*} i}\left(\mu^{t}\right)=0
$$

This completes part (1) of the proof. Repeating this construction for all $\left(i^{*}, j^{*}\right)$ such that $\mu\left(i^{*}, j^{*}\right) \neq \bar{\mu}\left(i^{*}, j^{*}\right)$ leads to the sequence $\bar{\mu}^{t}$ which converges to $\bar{\mu}$.

(2) Next we assume that $\operatorname{supp}(\bar{\mu}) \subsetneq \operatorname{supp}(\mu)$. Let $\left(i^{*}, j^{*}\right)$ be such that $\left(i^{*}, j^{*}\right) \in \operatorname{supp}(\mu)$ and $\left(i^{*}, j^{*}\right) \notin \operatorname{supp}(\bar{\mu})$. Let $\left(\varepsilon_{k}\right)_{k=1}^{\infty} \in \mathbb{R}^{N}$ be a sequence such that

$$
\lim _{k \rightarrow \infty} \varepsilon_{k}=0
$$

By part (1) there exists for every $\varepsilon_{k}>0$ a sequence $\mu_{\varepsilon_{k}}^{t}$

$$
\mu_{\varepsilon_{k}}^{t}(i, j):=\left\{\begin{array}{cc}
\frac{\varepsilon_{k}}{\mu(i, j)} \cdot \mu^{t^{\prime}(t)}(i, j) & \text { if }(i, j)=\left(i^{*}, j^{*}\right) \\
\mu^{t^{\prime}(t)}(i, j) & \text { else }
\end{array}\right.
$$

which converges to

$$
\mu_{\varepsilon_{k}}(i, j):=\left\{\begin{array}{cc}
\varepsilon_{k} & \text { if }(i, j)=\left(i^{*}, j^{*}\right) \\
\mu(i, j) & \text { else. }
\end{array}\right.
$$

Now the sequence $\left(\mu_{\varepsilon_{1}}^{l}\right)_{t=1}^{\infty}$ with limit $\bar{\mu}_{\varepsilon}:=\lim _{t \rightarrow \infty} \mu_{\varepsilon_{t}}^{t}$ is a sequence of correlated $t$-equilibria and has the property

$$
\operatorname{supp}\left(\bar{\mu}_{\varepsilon}\right)=\operatorname{supp}(\mu) \backslash\left(i^{*}, j^{*}\right) .
$$

This completes the proof of the theorem. 


\title{
Chapter 6
}

\section{Generalized stochastic dominance}

\begin{abstract}
A familiar way to order probability distributions on a set of alternatives is to use (first or higher degree) stochastic dominance. A typical property of such an (incomplete) ordering or preference is that a positive probability on a bad alternative cannot be compensated by putting high probabilities on better alternatives. For instance, a probability distribution that puts positive probability on the worst alternative can never stochastically dominate another probability distribution that puts less probability on that alternative, not even by putting all remaining probability on a best alternative.

In this chapter we study and characterize this typical property, which we call bad outcome aversion (BOA). Specifically, we consider incomplete preferences over lotteries on a finite set of alternatives and assume the classical conditions of (von Ncumann and Morgenstern) independence and continuity, so that the 'multi-expected utility' theorem of [Dubra et al, 2004] applies. This result characterizes such preferences in terms of representing closed and convex sets of functions. Our main result (Theorem 6.14) characterizes BOA for suclı preferences in terms of specific elements contained in these representing sets of functions. Thus, we offer a broad generalization of stochastic dominance preferences. The literature on stochastic dominance is vast: see [Levy, 1992] for an overview of theory and applications. For this chapter, in particular [Fishburn, 1976] is of interest. One other direct source of inspiration is the previous chapter of this dissertation on the application of stochastic dominance preferences in twoperson non-cooperative games, in turn, building on [Fishburn, 1978].
\end{abstract}

Section 6.1 formulates the model and recalls the multi-expected utility characterization of Dubra et al. (2004). Section 6.2 studies stochastic dominance preferences and in particular adapts Fishburn (1976) to our context. The bad outcome aversion condition is introduced in Section 6.3, which also contains our main results. Section 4 considers the game theoretic implications of this survey 
and, particularly. shows that the limit results we obtained for stochastic dominance relations in Chapter 4 generally hold for preference relations satisfying a set of axioms and BOA in particular.

\subsection{Preliminaries}

Let $X:=\left\{x_{1} \ldots, x_{n}\right\}$, where $n \geq 3$, be a finite set of alternatives and let $\Delta(X)$ denote the sot of probability distributions (lotteries) over $X$. We also use the letters $x, y \ldots$ to denote elements of $X$. A preference $\succeq$ is a reflexive and transitive binary relation on $\Delta(X)$. If $(p, q) \in \succeq$ we say that $p$ is (weakly) preferred over $q$. Instead of $(p, q) \in \succeq$ we often use the notation $p \succeq q$. We write $p \succ q$ if $p \succeq q$ and $q \succeq p$, and $p \sim q$ if $p \succeq q$ and $q \succeq p$. For $p \in \Delta(X)$ and $i \in\{1, \ldots, n\}, p_{i}$ denotes the probability that $p$ assigns to $x_{i}$. The degenerate lottery that assigns probability one to the alternative $x \in X$ is identified with $x$. Observe that we do not require completeness of $\succeq$.

The following possible conditions on $\succeq$ are well-known.

Axiom 6.1 (Independence) For all $p, q, r \in \Delta(X)$ and $0 \leq \lambda \leq 1$,

$$
p \succeq q \Rightarrow \lambda p+(1-\lambda) r \succeq \lambda q+(1-\lambda) r .
$$

Axiom 6.2 (Continuity) For all $q \in \Delta(X)$, the sets $\{p \in \Delta(X) \mid p \succeq q\}$ and $\{p \in \Delta(X) \mid q \succeq p\}$ are closed in $\Delta(X)$.

Let $U \subseteq \mathbb{R}^{X}$ be a set of real-valued functions on $X$. For $u \in \mathbb{R}^{X}$ and a lottery $p \in \Delta(X)$ denote by

$$
\mathbb{E}_{u}(p):=\sum_{i=1}^{n} p_{i} u\left(x_{i}\right)
$$

the expectation of $p$ under $u$. We say that $U$ represents the preference $\succeq$ if for all $p, q \in \Delta(X)$,

$$
p \succeq q \Leftrightarrow \mathbb{E}_{u}(p) \geq \mathbb{E}_{u}(q) \text { for all } u \in U .
$$

The following 'multi-expected utility' theorem follows from Dubra et al. (2004) and generalizes the familiar von Neumann-Morgenstern expected utility theorem to incomplete preferences.

Theorem 6.3 Let $\succeq$ be a preference. Then $\succeq$ satisfies indcpendence and continuity if and only if there is a closed and convex set $U \subseteq \mathbb{R}^{X}$ that represents $\succeq$.

\subsection{Stochastic dominance}

First degree stochastic dominance is a well-known example of a preference to which Theorem 6.3 applies. For any permutation $\pi$ of $\{1, \ldots, n\}$, the first degree 
stochastic dominance preference $\succeq_{\pi}$ is defined by

$$
p \succeq_{\pi} q \Leftrightarrow \sum_{i=1}^{j} p_{\pi(i)} \leq \sum_{i=1}^{j} q_{\pi(i)} \text { for all } j=1, \ldots, n
$$

for all $p, q \in \Delta(X)$. Note that $\succeq_{\pi}$ strictly orders all alternatives of $X$, specifically, $x_{\pi(n)} \succ_{\pi} \ldots \succ_{\pi} x_{\pi(1)}$. So first degree stochastic dominance preferences are complete on degenerate lotteries. Conversely, if a preference satisfies independence and strictly orders all alternatives of $X$, then it contains a first degree stochastic dominance preference. To show this formally, we introduce the following condition. This condition says that, ceteris paribus, shifting probability to a better alternative makes a lottery preferable.

Axiom 6.4 (Improvement) For all $p, q \in \Delta(X)$ and all $x, y \in X$ such that (i) $x \succeq y$, (ii) $p(z)=q(z)$ for all $z \in X \backslash\{x, y\}$, and (iii) $p(x)>q(x)$ and (hence) $p(y)<q(y)$, we have $p \succeq q$.

Lemma 6.5 Let $\succeq$ satisfy independence. Then $\succeq$ satisfies improvement.

Proof. Let $p, q, x, y$ satisfy the conditions in the statement of the improvement axiom. Let $\varepsilon:=p(x)-q(x)>0$. Define $\tilde{p} \in \Delta(X)$ by

$$
\tilde{p}(z)= \begin{cases}\frac{1}{1-\varepsilon} p(z) & \text { if } z \neq x, y \\ \frac{1}{1-\varepsilon} q(x) & \text { if } z=x \\ \frac{1}{1-\varepsilon} p(y) & \text { if } z=y\end{cases}
$$

Then $x \succeq y$ implies $p=\varepsilon x+(1-\varepsilon) \tilde{p} \succeq \varepsilon y+(1-\varepsilon) \tilde{p}=q$ by independence.

Lemma 6.6 Let $\succeq$ be a preference with either $x \succeq y$ or $y \succeq x$ for all $x, y \in X$, $x \neq y$. Then:

(i) There is a permutation $\pi$ of $\{1, \ldots, n\}$ such that $x_{\pi(n)} \succ \ldots \succ x_{\pi(1)}$.

(ii) $\succeq$ satisfies improvement if and only if $\succeq_{\pi} \subseteq \succeq$.

Proof. Part (i) is obvious. For the 'if' part of (ii), consider $p, q, x, y$ satisfying the conditions in the statement of the improvement axiom. In particular, $x=x_{\pi(i)}$ and $y=x_{\pi(j)}$ for some $i>j$. This implies $p \succeq_{\pi} q$, and therefore $p \succeq q$.

For the 'only if' part, for simplicity assume $\pi$ is identity, so that $x_{n} \succ \ldots \succ$ $x_{1}$. Let $p, q \in \Delta(X)$ such that $p \neq q$ and $p \succeq_{\pi} q$. Then, by definition,

$$
\sum_{i=1}^{j} p_{i} \leq \sum_{i=1}^{j} q_{i} \text { for all } j=1, \ldots, n .
$$


Hence, for $i^{*}:=\min \left\{i \mid p_{i} \neq q_{i}\right\}$ we have $q_{i^{*}}-p_{i^{*}}>0$. For $i^{*} \leq k \leq n$ define $q^{k} \in \Delta(X)$ by

$$
q_{i}^{k}:=\left\{\begin{array}{cl}
p_{i} & \text { if } i \leq k-1 \\
q_{k}+\sum_{l=0}^{k-1}\left(q_{l}-p_{l}\right) & \text { if } i=k \\
q_{i} & \text { if } i \geq k+1
\end{array}\right.
$$

By $(6.1), q^{k}$ is well-defined, and $q^{i^{*}}=q, q^{n}=p$. Improvement and $\sum_{i=1}^{k} p_{i} \leq \sum_{i=1}^{k} q_{i}$ imply $q^{k+1} \succeq q^{k}$ for each $i^{*} \leq k \leq n-1$, so $p \succeq q$ by transitivity of $\succeq$.

Lemmas 6.5 and 6.6 imply the following corollary.

Corollary 6.7 Let the preference $\succeq$ satisfy independence. If there is a permutation $\pi$ of $\{1, \ldots, n\}$ such that $x_{\pi(n)} \succ \ldots \succ x_{\pi(1)}$, then $\succeq_{\pi} \subseteq \succeq$.

For both illustrative purposes and later reference we now extend first degrce stochastic dominance to $t$-degree stochastic dominance, for any $t \in \mathbb{R}$ with $t \geq 1$. This extension adlapts Fishburn (1976) to our setting. We start with the following definition.

Definition 6.8 Let $t \in \mathbb{R}, t \geq 1$. The $n \times$ n-matrix $A_{t}$ is defined by $\left(a_{t}\right)_{i j}:=0$ for all $i, j \in\{1, \ldots, n\}$ with $i>j$ and by

$$
\left(a_{t}\right)_{i j}:=\frac{\Gamma(t+j-i)}{(j-i) ! \Gamma(t)}
$$

for all $i, j \in\{1, \ldots, n\}$ with $i \leq j$. Here, $\Gamma$ denotes the $\Gamma$-function

$$
\Gamma(x)=\int_{0}^{\infty} s^{x-1} e^{-s} d s .
$$

The proof of the following lemma is given in the Appendix to this chapter.

Lemma 6.9 For all $t, t^{\prime} \in \mathbb{R}, t, t^{\prime} \geq 1$, we have

$$
A_{t+t^{\prime}}=A_{t} A_{t^{\prime}}
$$

Lemma 6.9 implies that, if we define $A:=A_{1}$, then we can obtain $A_{t}$ as $A$ to the power $t$ for all $t \geq 1$, and therefore we write $A^{t}$ instead of $A_{t}$ in the remainder of this monograph.

For $t \geq 1$ wo define the $t$-degree stochastic dominance preference $\succeq_{t}$ by

$$
p \succeq_{t} q \Leftrightarrow p A^{t} \leq q A^{t}
$$

for all $p, q \in \Delta(X)$, where the inequality is coordinate-wise. It is not hard to verify that $x_{n} \succ_{t} \ldots \succ_{t} x_{1}$, thus $\succeq_{t}$ strictly orders the elements of $X{ }^{1}$ Also, $\succeq_{1}$

\footnotetext{
${ }^{1} t$-degree stochastic dominance can also be defined for different orderings of the alternatives in $X$, but for simplicity we restrict all definitions here to the ordering $x_{n} \succ_{l} \ldots \succ_{l} x_{1}$.
} 
is the first degree stochastic clominance preference associated with this ordering of the elements of $X$, i.e., $\succeq_{1}$ is equal to $\succeq_{\pi}$ for $\pi$ being the identity.

The following lemma collects some further facts about $t$-degree stochastic dominance from the previous chapter that we need for out purposes here. We omit the (straightforward) proofs and only note that these facts extend similar observations about the discrete case $t \in \mathbb{N}$.

Lemma 6.10 (i) For all $t, t^{\prime} \in \mathbb{R}$ with $t \geq t^{\prime} \geq 1$, we have $\succeq_{t^{\prime}} \subseteq \succeq_{t}$.

(ii) For all $p, q \in \Delta(X)$ with $p \neq q$, if $p_{i^{*}}<q_{i^{*}}$ where $i^{*}:=\min \left\{i \mid p_{i} \neq q_{i}\right\}$, then there is a $t^{\prime} \geq 1$ such that $p \succeq_{t} q$ for all $t \geq t^{\prime}$.

This lemma implies that $t$-degree stochastic dominance preferences becomes more complete inclusion-wise if $t$ becomes large, and that any pair of lotteries is eventually ordered.

The (complete) lexicographic preference $\succeq_{L M I}$ is defined by

$$
p \succeq_{L M} q \Leftrightarrow p=q \text { or } p_{i^{*}}<q_{i^{*}}
$$

for all $p, q \in \Delta(X)$, where as before $i^{*}:=\min \left\{i \mid p_{i} \neq q_{i}\right\}$.

The prefercnces $\succeq_{t}$ have the property that as $t$ increases a (potentially small) probability that is put on a bad outcome must be compensated by an increasing weight on a good outcome. In the limit compensation is impossible. This is also expressed by the following corollary to Lemma 6.10.

Corollary 6.11 For all $t \in \mathbb{R}, t \geq 1$, it holds lhat $\succeq_{t} \subseteq_{L M}$, and

$$
\bigcup_{t=1}^{\infty} \succeq_{t}=\succeq_{L M I} .
$$

Lemma 6.10 and Corollary 6.11 also imply that for any $p, q \in \Delta(X)$ with $p \neq q$ and satisfying the condition on the right hand side of (6.2), we have $q Z_{t} p$ for all $t \geq 1$. Hence, this is a characteristic property of stochastic dominance preferences. In the next section we formalize this property as an axiom of 'bad outcome aversion' and characterize preferences that, beside continuity and independence, satisfy this axiom.

\subsection{Bad outcome aversion}

The following axiom captures and generalizes a basic property of stochastic dominance preferences, as explained at the end of the preceding section.

Axiom 6.12 (Bad outcome aversion, BOA) For all $p, q \in \Delta(X)$ and all $x \in X$, if $p(x)>q(x)$ and $x \nsucceq z$ for all $z \in X \backslash\{x\}$ for which $p(z) \neq q(z)$, then $p \nsucceq q$. 
'The interpretation of this axiom is as follows. Think of $x$ as a 'bad' alternative, on which $p$ puts more weight than $q$. 'Then the axiom says that this can never be compensated by the weights put by $p$ on alternatives that are better or at least not worse than $x$.

Clearly, by Lemma 6.10 and Corollary 6.11, stochastic dominance preferences and the lexicographic preference $\succeq_{L M}$ satisfy BOA. More generally, for each permutation $\pi$ of $\{1, \ldots, n\}$ define the lexicographic preference $\succeq_{L M, \pi}$ by

$$
p \succeq_{L, M, \pi} q \Leftrightarrow p=q \text { or } p_{\pi\left(i^{*}\right)}<q_{\pi\left(i^{*}\right)}
$$

for all $p . q \in \Delta(X)$, where $i^{*}:=\min \left\{i \mid p_{\pi(i)} \neq q_{\pi(i)}\right\}$. The following proposition says that a preference satisfies BOA and therc there are no indifferences betwen the alternatives in $X$ if and only if it is a subset of some lexicographic preference.

Proposition 6.13 Let $\succeq$ be a preference. Then the following statements are equivalent.

(i) $\succeq$ satisfies $B O A$, and $x \neq y$ for all $x, y \in X$ with $x \neq y$.

(ii) $\succeq \subseteq \succeq_{L M, \pi}$ for some permutation $\pi$ of $\{1, \ldots, n\}$.

Proof. The implication (ii) $\Rightarrow$ (i) is obvious. For the converse implication, take a permutation $\pi$ such that for all $i, j \in\{1, \ldots, n\}$, if $i>j$ then $x_{\pi(j)} \nsucceq$ $x_{\pi(i)}$. (Such a permutation can be seen to exist since $x \neq y$ for all $x \neq y$ in $X$, although it may not be unique.) Let $p, q \in \Delta(X)$ with $p \neq q$ and $p \succeq q$. Let $i^{*}:=\min \left\{i \mid p_{\pi(i)} \neq q_{\pi(i)}\right\}$. Since $x_{\pi\left(i^{*}\right)} \nsucceq x_{\pi(i)}$ for all $i>i^{*}$, BOA of $\succeq$ implies $p_{\pi\left(i^{*}\right)}<q_{\pi\left(i^{*}\right)}$. Thus, $p \succeq_{L M, \pi} q$.

We now characterize BOA for preferences that satisfy independence and contimuity and that strictly order all elements of $X$, by using the multi-expected utility theorem, Theorem 6.3. More precisely, we show that such a preference satisfies $\mathrm{BOA}$ if and only if the representing class of functions contains specific elements.

Theorem 6.14 Lot the preference $\succeq$ satisfy independence and continuity, and suppose $x_{n} \succ x_{n-1} \succ \ldots \succ x_{1}$. Let $U \subseteq \mathbb{R}^{X}$ represent $\succeq$. Then $\succeq$ satisfies $B O A$ if and only if for each $i=1, \ldots, n-1$ there is a sequence $\left(u^{k}\right)_{k \in \mathbb{N}}, u^{k} \in U$ and $u^{k}\left(x_{i}\right)<u^{k}\left(x_{n}\right)$ for each $k \in \mathbb{N}$, such that

$$
\lim _{k \rightarrow \infty} \frac{u^{k}\left(x_{n}\right)-u^{k}\left(x_{i+1}\right)}{u^{k}\left(x_{n}\right)-u^{k}\left(x_{i}\right)}=0 .
$$

Proof. We may normalize any $u \in U$ such that $u\left(x_{1}\right)=0$ and $u\left(x_{n}\right)=1$.

For the 'if' part, let $p, q, x$ satisfy the conditions in the statement of BOA, so $x=x_{i}$ for some $i \in\{1, \ldots, n-1\}$. Let $\left(u^{k}\right)_{k \in \mathbb{N}}$ be a sequence with $u^{k} \in U$ and $u^{k}\left(x_{i}\right)<u^{k}\left(x_{n}\right)$ for aach $k \in \mathbb{N}$ such that (6.4) is satisfied. Without 
loss of generality we may assume that the sequence $\left(u^{k}\right)_{k \in \mathbb{N}}$ converges. With $\alpha:=\sum_{j=1}^{i-1} q_{j}=\sum_{j=1}^{i-1} p_{j}$ we can write

$$
\lim _{k \rightarrow \infty} \mathbb{E}_{u^{k}}(q)=\lim _{k \rightarrow \infty} \sum_{j=1}^{i-1} q_{j} u^{k}\left(x_{j}\right)+q_{i} u^{k}\left(x_{i}\right)+\left(1-q_{i}-\alpha\right) u^{k}\left(x_{i+1}\right)
$$

and

$$
\lim _{k \rightarrow \infty} \mathbb{E}_{u^{k}}(p)=\lim _{k \rightarrow \infty} \sum_{j=1}^{i-1} p_{j} u^{k}\left(x_{j}\right)+p_{i} u^{k}\left(x_{i}\right)+\left(1-p_{i}-\alpha\right) .
$$

We claim that for $k$ sufficiently large, $\mathbb{E}_{u^{k}}(q)>\mathbb{E}_{u^{k}}(p)$. To show this, it is sufficient to prove that

$$
q_{i} u^{k}\left(x_{i}\right)+\left(1-q_{i}-\alpha\right) u^{k}\left(x_{i}+1\right)>p_{i} u^{k}\left(x_{i}\right)+\left(1-p_{i}-\alpha\right)
$$

or, equivalently

$$
\left(1-q_{i}-\alpha\right)\left[1-u^{k}\left(x_{i+1}\right)\right]<\left(p_{i}-q_{i}\right)\left[1-u^{k}\left(x_{i}\right)\right]
$$

for $k$ sufficiently large. This, however, follows by (6.4). So $p \nsucceq q$.

For thr converse, assume that $\succeq$ satisfies BOA. Fix $i \in\{1, \ldots, n-1\}$. Fix $0<\pi<1$, consider the lottery $p=\pi x_{\imath}+(1-\pi) x_{i+1}$, and for each $0<\epsilon<1-\pi$ consider the lot.tery $p^{f}=(\pi+\epsilon) x_{i}+(1-\pi-\epsilon) x_{n}$. By BOA, $p^{c} \nsucceq p$, hence there is a $u^{\epsilon} \in U$ such that $u^{\epsilon}(p)>u^{\epsilon}\left(p^{\epsilon}\right)$, i.e.,

$$
(1-\pi)\left(1-u^{\epsilon}\left(x_{i+1}\right)\right)<\varepsilon\left(1-u^{\epsilon}\left(x_{i}\right)\right) .
$$

This implies the existence of a sequence $\left(u^{k}\right)_{k \in \mathbb{N}}$ with $u^{k} \in U$ and $u^{k}\left(x_{i}\right)<$ $u^{k}\left(x_{n}\right)$ for each $k \in \mathbb{N}$ such that $(6.4)$ is satisfied.

We conclude this section with some remarks and a corollary for the case of three alternatives.

Remark 6.15 A consequence of Theorem 6.14 is that, under the additional conditions in the theorem, BOA implies incompleteness of the preference. This is so because a complete preference is represented by a unique $0-1$ normalized function and, thus, a sequence satisfying (6.4) cannot exist for every $i$.

Remark 6.16 If, in Theorem 6.14, for some $i \in\{1, \ldots, n-1\}$ there is a function $u \in U$ with $u\left(x_{i}\right)<u\left(x_{i+1}\right)=\ldots=u\left(x_{n}\right)$, then (6.4) can be trivially satisfied by taking $u^{k}=u$ for all $k \in \mathbb{N}$. For example, let $n=4$ and consider the set of funclions

$$
U=\operatorname{conv}\left\{(0, \eta, \eta(2-\eta), 1) \in \mathbb{R}^{4} \mid 0 \leq \eta \leq 1\right\}
$$

where 'conv' denotes the convex hull operator. This set is closed and convex. It contains the elements $(0,1,1,1)$ and (e.g.) $\left(0, \frac{1}{2}, \frac{1}{2}, 1\right)$, so that (6.4) is satisfied for $i=1$ and $i=3$. It does not contain an element of the form $(0, \alpha, 1,1)$ for some $0 \leq \alpha<1$, but (e.g.) the sequence $\left(0, \frac{k}{k+1}, \frac{k(k+2)}{(k+1)^{2}}, 1\right){ }_{k \in \mathbb{N}}$ satisfies (6.4) for $i=2$. It follows that the preference represented by $U$ satisfies $B O A$. 
Remark 6.17 Theorem 6.14 applies in particular to stochastic dominance preferences $\succeq_{t}, t \in \mathbb{R}, t \geq 1$. In that case for the sel $U$ we can take the convex hull of the columns of the matrix $-A^{t}$. This is a case in which for each $i=1, \ldots, n-1$ there is a function $u$ with $u\left(x_{i}\right)<u\left(x_{i+1}\right)=\ldots=u\left(x_{n}\right)$ as in Remark 6.16, namely the $i$-th column of $-A^{t}$.

Remark 6.18 For the case of three alternatives the consequences of Theorem 6.14 are as follows. Since we can assume that every $u \in U$ has the form $(0, \alpha, 1)$, the theorem applied for $i=1$ implies $(0,1,1) \in U$ and the theorem applied for $i=2$ implies $(0, \alpha, 1) \in U$ for some $\alpha<1$. Let $\alpha^{*}:=\inf \{\alpha \mid(0, \alpha, 1) \in U\}$, then convexity and closedness of $U$ imply that $U$ is the convex hull of $(0,1,1)$ and $\left(0, \alpha^{*}, 1\right)$. Consider, on the other hand, the $3 \times 3-$ matrix $-A^{t}$ and normalize its columns. This results in the matrix

$$
\left(\begin{array}{ccc}
0 & 0 & 0 \\
1 & \frac{t-1}{t} & \frac{t}{t+2} \\
1 & 1 & 1
\end{array}\right)
$$

implying that the class of functions $U^{t}$ representing $\succeq_{t}$ is the convex hull of $(0,1,1)$ and $(0,(t-1) / t, 1)$ if $t \geq 2$ and of $(0,1,1)$ and $(0, t /(t+2), 1)$ if $t \leq 2$. In turn, this implies that $\succeq$ coincides with $\succeq_{t}$, where $t=1 /\left(1-\alpha^{*}\right)$ if $\alpha^{*} \geq / 2$ and $t=2 \alpha^{*} /\left(1-\alpha^{*}\right)$ if $\alpha^{*} \leq 1 / 2$. Thus, we have the following corollary to Theorem 6.14.

Corollary 6.19 Let $X=\left\{x_{1}, x_{2}, x_{3}\right\}$ and let $\succeq$ satisfy independence, continuity, and BOA. Let $x_{3} \succ x_{2} \succ x_{1}$. Then $\succeq=\succeq_{t}$ for some $t \in \mathbb{R}, t \geq 1$. Actually the proof of this corollary is given in the previous remark. We also give an alternative proof below (Lemma 6.21).

Lemma 6.20 Let $\succeq$ be a preference relation over $\Delta(X)$, where $X=\left\{x_{1}, x_{2}, x_{3}\right\}$. Furthermore, let $\succeq$ satisfy Continuity and Improvement. Then there exists an $x^{*}(\succeq) \in(0,1]$ such that

$$
\begin{aligned}
& (0,1,0) \succeq(x, 0,1-x) \forall x \geq x^{*}(\succeq) \text { and } \\
& (0,1,0) \nsucceq(x, 0,1-x) \forall x<x^{*}(\succeq) .
\end{aligned}
$$

Proof. Let

$$
X:=\{x \in[0,1] \mid(0,1,0) \succeq(x, 0,1-x)\} .
$$

By Improvement, $1 \in X$ and $0 \notin X$. Furthermore, if $x \in X$ and $y>x$, then $y \in X$. Let $x^{*}:=\inf X$. Then, by continuity it holds that $0<x^{*} \leq 1$.

Lemma 6.21 A preference relation $\succeq$ over $\Delta(X), X=\left\{x_{1}, x_{2}, x_{3}\right\}$, satisfies Independence, Continuity, Bad Outcome Aversion and Improvement if and only if there cxists a $t \in \mathbb{R}, t \geq 1$ such that $\succeq$ is induced by $t$-th order stochastic dominance. Furthermore, if $x^{*}$ is as in Lemma 6.20, it holds that

$$
t= \begin{cases}\frac{1}{x^{*}} & \frac{1}{2}<x^{*} \leq 1 \\ \frac{2-2 x^{*}}{x^{*}} & 0<x^{*} \leq \frac{1}{2}\end{cases}
$$


Proof. First we prove the following claim

Claim 6.22 Let $p:=(0,1,0)$ and $q:=(x, 0,1-x), x \in(0,1)$. Then $p \succeq_{t} q$ if and only if

$$
x>\left\{\begin{array}{cc}
\frac{2}{t+2} & \text { if } t \geq 2 \\
\frac{1}{t} & \text { if } 1 \leq t<2 .
\end{array}\right.
$$

Proof of Claim. For three states it holds that

$$
A^{t}=\left(\begin{array}{ccc}
1 & t & \frac{1}{2} t(t+1) \\
0 & 1 & t \\
0 & 0 & 1
\end{array}\right)
$$

and hence,

$$
\begin{aligned}
& p A^{t}=(0,1, t) \\
& q A^{t}=\left(x, t x, 1-x+\frac{1}{2} t(t+1) x\right),
\end{aligned}
$$

yielding the conditions

$$
\begin{aligned}
0 & \leq x \\
1 & \leq t x \\
t & \leq 1-x+\frac{1}{2} t(t+1) x
\end{aligned}
$$

where at least onc of the inequalities is strict. Solving this system leads to the statement of the claim 4 .

Now we prove $\succeq_{t}=\succeq$. Let $p:=\left(p_{1}, p_{2}, p_{3}\right)$ and $q:=\left(q_{1}, q_{2}, q_{3}\right)$ such that $p \neq q$. Distinguish the following cases.Let $p_{2}>q_{2}, p_{1}<q_{1}, p_{3}<q_{3}$. Define

$$
\begin{aligned}
\lambda & :=p_{2}-q_{2} \\
x^{\prime} & :=\frac{q_{1}-p_{1}}{p_{2}-q_{2}}=\frac{q_{1}-p_{1}}{\lambda} .
\end{aligned}
$$

Now,

$$
\begin{aligned}
& \left(p_{1}, p_{2}, p_{3}\right)=\lambda(0,1,0)+(1-\lambda)\left(\frac{p_{1}}{1-\lambda}, \frac{q_{2}}{1-\lambda}, \frac{p_{3}}{1-\lambda}\right) \\
& \left(q_{1}, q_{2}, q_{3}\right)=\lambda\left(x^{\prime}, 0,1-x^{\prime}\right)+(1-\lambda)\left(\frac{p_{1}}{1-\lambda}, \frac{q_{2}}{1-\lambda}, \frac{p_{3}}{1-\lambda}\right)
\end{aligned}
$$

and hence, by Independence,

$$
\left(p_{1}, p_{2}, p\right) \succeq^{*}\left(q_{1}, q_{2}, q_{3}\right) \Longleftrightarrow(0,1,0) \succeq^{*}\left(\frac{q_{1}-p_{1}}{p_{2}-q_{2}}, 0,1-\frac{q_{1}-p_{1}}{p_{2}-q_{2}}\right),
$$

where

$$
\succeq^{*} \in\left\{\succeq, \succeq_{t}\right\}
$$


By Lemma 6.20 there exists a $x^{*}(\succeq)$ such that (6.5) holds. By Bad outcome aversion

$$
\left(x^{\prime}, 0,1-x^{\prime}\right) \nsucceq(0,1,0)
$$

for all $x^{\prime}>0$. Hence, Claim 6.22 implics that for

$$
t=\left\{\begin{array}{cc}
\frac{1}{x^{\prime}} & \frac{1}{2}<x^{\prime} \leq 1 \\
\frac{2-2 x^{\prime}}{x^{\prime}} & 0<x^{\prime} \leq \frac{1}{2}
\end{array}\right.
$$

it holds that

$$
(0,1,0) \succeq\left(x^{\prime}, 0,1-x^{\prime}\right) \Longleftrightarrow(0,1,0) \succeq_{t}\left(x^{\prime}, 0,1-x^{\prime}\right)
$$

Let $p_{2}>q_{2}, p_{1}<q_{1}, p_{3}>q_{33}$. Define

$$
\lambda:=q_{2}-p_{2}
$$

Then

$$
\begin{aligned}
\left(p_{1}, p_{2}, p_{3}\right) & =\lambda\left(0, \frac{p_{2}-q_{2}}{\lambda}, \frac{p_{3}-q_{3}}{\lambda}\right)+(1-\lambda)\left(\frac{p_{1}}{1-\lambda}, \frac{q_{2}}{1-\lambda}, \frac{q_{3}}{1-\lambda}\right) \\
\left(q_{1}, q_{2}, q_{3}\right) & =\lambda(1,0,0)+(1-\lambda)\left(\frac{p_{1}}{1-\lambda}, \frac{q_{2}}{1-\lambda}, \frac{q_{3}}{1-\lambda}\right) .
\end{aligned}
$$

Now $p \succeq q$ holds by Independence and Improvement. Since both $\succeq$ and $\succeq_{t}$ satisfy Independence and Improvement, it holds that $p \succeq^{*} q$ for $\succeq^{*} \in\left\{\succeq, \succeq_{t}\right\}$. Let $p_{2}>q_{2}, p_{1}>q_{1}$. Then $q \succeq^{*} p$ for $\succeq^{*} \in\left\{\succeq, \succeq_{t}\right\}$. Normalization implies $q_{3}>p_{3}$.
Define

$$
\lambda:=q_{3}-p_{3}
$$

Then,

$$
\begin{aligned}
\left(p_{1}, p_{2}, p_{3}\right) & =\lambda\left(\frac{p_{1}-q_{1}}{\lambda}, \frac{p_{2}-q_{2}}{\lambda}, 0\right)+(1-\lambda)\left(\frac{q_{1}}{1-\lambda}, \frac{q_{2}}{1-\lambda}, \frac{p_{3}}{1-\lambda}\right) \\
\left(q_{1}, q_{2}, q_{3}\right) & =\lambda(0,0,1)+(1-\lambda)\left(\frac{q_{1}}{1-\lambda}, \frac{q_{2}}{1-\lambda}, \frac{p_{3}}{1-\lambda}\right) .
\end{aligned}
$$

Now, $q \succeq p$ by Independence and Improvement, implying that $q \succeq^{*} p$ for $\succeq^{*}$ $\in\left\{\succeq, \succeq_{t}\right\}$. Let $p_{2}>q_{2}, p_{1}=q_{1}$. Then $q \succeq^{*} p$ for $\succeq^{*} \in\left\{\succeq \succeq_{t}\right\}$. In this case
we write

$$
\begin{aligned}
& \left(p_{1}, p_{2}, p_{3}\right)=q_{3}\left(0, \frac{p_{2}-q_{2}}{q_{3}}, \frac{p_{3}}{q_{3}}\right)+\left(1-q_{3}\right)\left(\frac{p_{1}}{1-q_{3}}, \frac{q_{2}}{1-q_{3}}, 0\right) \\
& \left(q_{1}, q_{2}, q_{3}\right)=q_{3}(0,0,1)+\left(1-q_{3}\right)\left(\frac{p_{1}}{1-q_{3}}, \frac{q_{2}}{1-q_{3}}, 0\right)
\end{aligned}
$$

and $q \succeq p$ by Independence and Improvement. Hence, $q \succeq^{*} p$ for $\succeq^{*} \epsilon$ $\left\{\succeq, \succeq_{t}\right\}$. Let $p_{2}=q_{2}$. Then $q \succeq^{*} p$ for $\succeq^{*} \in\left\{\succeq, \succeq_{t}\right\}$ if and only if $p_{1}<q_{1}$. 
Assume w.l.o.g. that $p_{1}>q_{1}$. Then

$$
\begin{aligned}
\left(p_{1}, p_{2}, p_{3}\right) & =q_{3}\left(\frac{p_{1}-q_{1}}{q_{3}}, 0, \frac{p_{3}}{q_{3}}\right)+\left(1-q_{3}\right)\left(\frac{q_{1}}{1-q_{3}}, \frac{p_{2}}{1-q_{3}}, 0\right) \\
\left(q_{1}, q_{2}, q_{3}\right) & =q_{3}(0,0,1)+\left(1-q_{3}\right)\left(\frac{q_{1}}{1-q_{3}}, \frac{p_{2}}{1-q_{3}}, 0\right)
\end{aligned}
$$

and $q \succeq p$ by Independence and Improvement. Thus, $q \succeq^{*} p$ for $\succeq^{*} \in\left\{\succeq, \succeq_{t}\right\}$.

This completes the proof.

We conclude this section with some remarks. Although there is no direct formal connection, bad outcome aversion is somewhat similar in flavor to risk aversion. It is well-known that first degree stochastic dominance has the set of all functions (respecting the order on the alternatives in $X$ ) as representing set - this can be seen, for instance, by considering the matrix $-A^{1}$ - and that for second degree stochastic dominance this is the set of all concave, 'risk averse' functions, i.c., the set of all functions exhibiting decreasing differences.

One could say that higher degree stochastic dominance corresponds to higher bad outcome aversion or, more generally, that $\succeq$ is more bad outcome averse than $\succeq$ if $\succeq_{1} \subseteq \succeq \subseteq \succeq^{\prime} \subseteq \succeq_{L M I}$, cf. Corollary 6.7 and Proposition 6.13. We can consider the previous chapter as an application of this. In the next section we show that the results in that chapter and in particular the asymptotic result remains true if players become 'more bad outcome averse' in the more general sense described above.

\subsection{Game theoretic implications}

\subsubsection{Best replies and equilibria}

We now consider a two-person gamc. Player 1 has pure strategy set $M:=$ $\{1, \ldots, m\}$ and player 2 has pure strategy set $N:=\{1, \ldots, n\}$. If player 1 plays the pure strategy $i \in M$, and player 2 plays the pure strategy $j \in N$ the outcome $x_{i j}$ results. Hence,

$$
X:=\left\{x_{i j} \mid i \in M, j \in N\right\}
$$

is the set of pure outcomes. We consider the case in which every player has a preference relation on $\Delta(X)$ that satisfics Independence, Continuity, Bad Outcome Aversion and Improvement. Hence, we consider games

$$
\Gamma=\Gamma\left(M, N, \succeq_{1}, \succeq_{2}\right) .
$$

Here, $\succeq_{1}$ and $\succeq_{2}$ denote the preference relations with which player 1 and 2 , respectively, evaluate probability distributions over $X$. We say that $p \in \Delta(M)$ is a $\succeq_{1}$-best reply to $q \in \Delta(N)$ if there is no $p^{\prime} \in \Delta(M)$ such that $p^{\prime} q \succeq_{1} p q$. Here, $p q$ is the probability distribution that $p$ and $q$ induce on pure outcomes and $\succeq_{1}$ is the preference relation by which player 1 evaluates probability distributions 
over these pure outcomes. We say that $(p, q)$ is a $\left(\succeq_{1}, \succeq_{2}\right)$-equilibrium if $p$ is a $\succeq_{1}$-best reply to $q$ and $q$ is a $\succeq_{2}$-best reply to $p$.

Since the preference relations $\succeq_{1}, \succeq_{2}$ are complete on $X$ they can be represented, respectively, by bijections $\sigma, \tau: M \times N \rightarrow\{1, \ldots, m n\}$. As an example player 1 prefers $x_{i j}$ to $x_{i^{\prime} j^{\prime}}$ if and only if $\sigma(i, j)>\sigma\left(i^{\prime}, j^{\prime}\right)$. By [Dubra et, al, 2004] there exists a closed and convex set $U$ such that

$$
p q \succeq_{1} p^{\prime} q \Longleftrightarrow \sum_{i=1}^{m} \sum_{j=1}^{n} p_{i} q_{j} u_{\sigma(i, j)} \geq \sum_{i=1}^{m} \sum_{j=1}^{n} p_{i}^{\prime} q_{j} u_{\sigma(i, j)}
$$

for all $u \in U$. Here, the vector $u_{\sigma}$ is such that

$$
u_{\sigma(i, j)}=u(\gamma) \text { such that } \sigma(i, j)=\gamma .
$$

Hence, $p$ is a $\succeq_{1}$-best reply to $q \in \Delta(N)$ if for every $p^{\prime} \in \Delta(M)$ there exists a $u \in U$ such that

$$
\sum_{i=1}^{m} \sum_{j=1}^{n} p_{i} q_{j} u_{\sigma(i, j)} \geq \sum_{i=1}^{m} \sum_{j=1}^{n} p_{i}^{\prime} q_{j} u_{\sigma(i, j)}
$$

Analogous objects are defined for player 2. The following Lemma is a clirect consequence of the expected utility representation.

Definition 6.23 Let $p \in \Delta(M)$. Then the set

$$
\operatorname{supp}(p):=\left\{i \in M \mid p_{i}>0\right\}
$$

is called the support of $p$.

Lemma 6.24 Let $p, p^{\prime} \in \Delta(M)$ and $q, q^{\prime} \in \Delta(N)$ such that $\operatorname{supp}\left(p^{\prime}\right) \subseteq \operatorname{supp}(p)$ and $\operatorname{supp}\left(q^{\prime}\right) \subseteq \operatorname{supp}(q)$. Then

(i) if $p$ is a $\succeq_{1}$-best reply to $q$, then $p^{\prime}$ is a $\succeq_{1}$-best reply to $q$;

(ii) if $q$ is $a \succeq_{2}$-best reply to $p$, then $q^{\prime}$ is a $\succeq_{1}$-best reply to $p$.

Proof. Apply the expected utility theorem.

Let the preference relations $\succeq_{1}$ and $\succeq_{2}$ be represented by closed and convex classes $U_{1}$ and $U_{2}$ respectively. Let $N E\left(u_{1}, u_{2}\right)$ be the set of all Nash equilibria of the game $\Gamma\left(M, N, u_{1}, u_{2}\right)$ and define

$$
N E\left(U_{1}, U_{2}\right):=\left\{\begin{array}{c}
(p, q) \in \Delta(M) \times \Delta(N) \mid \\
(p, q) \text { is a Nash equilibrium for some }\left(u_{1}, u_{2}\right) \in U_{\perp} \times U_{2}
\end{array}\right\}
$$

We call $(p, q) \in \Delta(M) \times \Delta(N)$ a $\left(\succeq_{1}, \succeq_{2}\right)$-equilibrium if $p$ is a $\succeq_{1}$-best reply to $q$ and $q$ is a $\succeq_{2}$-best reply to $p$. Define

$$
E\left(\succeq_{1}, \succeq_{2}\right):=\left\{\begin{array}{c}
(p, q) \in \Delta(M) \times \Delta(N) \mid \\
(p, q) \text { is a }\left(\succeq_{1}, \succeq_{2}\right) \text {-equilibrium }
\end{array}\right\}
$$

The definitions directly imply the following result.

Lemma 6.25 It holds that

$$
N E\left(U_{1}, U_{2}\right)=\bigcup_{u_{1} \in U_{1}} \bigcup_{u_{2} \in U_{2}} N E\left(u_{1}, u_{2}\right)=E\left(\succeq_{1}, \succeq_{2}\right)
$$




\subsubsection{Sequences of preference relations}

In this paragraph we construct sequences $\left(\succeq^{k}\right)_{k \in \mathbb{N}}$ of preference relations with, as $k$ increases, increasing aversion towards bad outcomes and investigate the game theoretic consequences if both players consider such sequences.

Definition 6.26 We call the sequence of preference relations $\left(\succeq^{k}\right)_{k \in \mathbb{N}}$ a complete preference sequence if

A. The preference relations $\succeq^{k}$ satisfy Independence, Continuity, Bad Outcome Aversion and Improvement for all $k \in \mathbb{N}$;

B. For all $k \in N$ it holds that

$$
\succeq^{k} \subseteq \succeq^{k+1}
$$

C. For every $p, p^{\prime} \in \Delta(M), q, q^{\prime} \in \Delta(N)$ therc exists a $k$ such that

$$
p q \succeq^{k} p^{\prime} q^{\prime} \text { or } p^{\prime} q^{\prime} \succeq^{k} p q
$$

First we prove the following lemma.

Lemma 6.27 Let $\left(\succeq^{k}\right)_{k \in \mathbb{N}}$ be a complete preference sequence and lel $\left(U^{k}\right)_{k \in N}$ be the sequence such that $U^{k}$ represents $\succeq^{k}$ for all $k \in \mathbb{N}$. Then for all $\gamma \in$ $\{1, \ldots, n-2\}$ there cxists a sequence $\left(u^{k}\right)_{k \in \mathbb{N}}$ with $u^{k} \in U^{k}$ for all $k$ and it holds that

$$
\lim _{k \rightarrow \infty} \frac{u_{\gamma+2}^{k}-u_{\gamma+1}^{k}}{u_{\gamma+2}^{k}-u_{\gamma}^{k}}=0 .
$$

Proof. Assume that the statement of the Lemma is false. Hence, there exist a $\gamma \in\{1, \ldots, n-2\}$ and an $\epsilon>0$ such that

$$
\frac{u_{\gamma+2}^{k}-u_{\gamma+1}^{k}}{u_{\gamma+2}^{k}-u_{\gamma}^{k}}>\epsilon
$$

for all $u^{k} \in U^{k}$ and all $k$. Now consider the following convex combination for $\alpha>0$

$$
\begin{aligned}
& \alpha u_{\gamma}^{k}+(1-\alpha) u_{\gamma+2}^{k} \\
= & \alpha\left(u_{\gamma}^{k}-u_{\gamma+2}^{k}\right)+u_{\gamma+2}^{k} \\
> & \frac{\alpha}{\epsilon}\left(u_{\gamma+1}^{k}-u_{\gamma+2}^{k}\right)+u_{\gamma+2}^{k} .
\end{aligned}
$$

Now, w.l.o.g. assume that $\epsilon<1$ and choose $\alpha:=\epsilon$. Then for all $k$ we obtain for all $u^{k} \in U^{k}$

$$
\alpha u_{\gamma}^{k}+(1-\alpha) u_{\gamma+2}^{k}>u_{\gamma+1}^{k},
$$

which contradicts Bad Outcome Aversion. 
If the context of a two-person gane we call the pair $\left(\succeq_{1}^{k}, \succeq_{2}^{k}\right)_{k \in \mathbb{N}}$ a two-persone complete prefercnce sequence if $\left(\succeq_{a}^{k}\right)_{k \in \mathbb{N}}$ is a complete preference sequence for $a=1,2$. The interpretation is that for every $k \in \mathbb{N}$ player 1 ranks his lotteries according to $\succeq_{1}^{k}$ and player 2 ranks his lotteries according to $\succeq_{2}^{k}$.

Lemma 6.28 Let $I \subseteq M, J \subseteq N$ and let $\left(\succeq_{1}^{k}, \succeq_{2}^{k}\right)_{k \in \mathbb{N}}$ be a two-persone complete preference sequence. Furthermore, let $\left(p^{k}, q^{k}\right) \in \Delta(I) \times \Delta(J)$ be a sequence such that $I=\operatorname{supp}\left(p^{k}\right)$ and $J=\operatorname{supp}\left(q^{k}\right)$ for all $k \in \mathbb{N}$. Then

(i) Let $p^{k}$ be $a \succeq_{1}^{k}$-best reply to $q^{k}$ for every $k \in \mathbb{N}$. Then, for every $i \in M$ there exists a $j \in J$ such that

$$
\sigma(i, j)<\sigma\left(i^{\prime}, j\right) \text { for all } i^{\prime} \in I \backslash\{i\}
$$

(ii) Let $q^{k}$ be $a \succeq_{2}^{k}$-best reply to $p^{k}$ for every $k \in \mathbb{N}$. Then, for every $j \in N$ there exists a $i \in I$ such that

$$
\tau(i, j)<\tau\left(i, j^{\prime}\right) \text { for all } j^{\prime} \in J \backslash\{j\}
$$

Proof. We only prove $(i)$; the proof of $(i i)$ is analogous. Assume that $(i)$ is false. Then there exists a $\hat{\imath} \in M$ such that for all $j \in J$ the set

$$
I_{j}:=\{i \in I \mid \sigma(i, j)<\sigma(\hat{\imath}, j)\} \neq \varnothing
$$

For every $j \in J$ pick a $i_{j} \in I_{j}$. Let

$$
\bar{I}:=\left\{i_{j} \mid j \in J\right\}
$$

and let $s:=|\bar{I}|$. We define the mixed strategy $\hat{p}$ by

$$
\hat{p}_{i}:= \begin{cases}\frac{1}{s} & i \in \bar{I} \\ 0 & \text { else. }\end{cases}
$$

Since $\bar{I} \subseteq I$ lemma 6.24 implies that $\hat{p}$ is a $\succeq_{1}^{k}$-best reply to $q^{k}$ for all $k \in \mathbb{N}$. We show that for $k$ large enough

$$
e_{\hat{\imath}} q^{k} \succeq_{1}^{k} \hat{p} q^{k}
$$

which would contradict the fact that $\hat{p}$ is a $\succeq_{1}^{k}$-best reply to $q^{k}$. Hence, we are left to show (6.6) for $k$ large enough. For every $j \in J$ define

$$
i_{j}^{*}:=\min \left\{\gamma \mid\left(\hat{p} e_{j} \sigma\right)_{\gamma} \neq\left(e_{\hat{\imath}} e_{j} \sigma\right)_{\gamma}\right\}
$$

Then by definition of $I_{j}$ and $\bar{I}$ it holds that

$$
\left(\hat{p} c_{j} \sigma\right)_{i_{j}^{*}}>\left(e_{\hat{\imath}} c_{j} \sigma\right)_{i_{j}^{*}}
$$


Bad Outcome Aversion implies $\hat{p} e_{j} \Varangle_{1}^{k} e_{i} e_{j}$ for all $k$ and hence, since $\left(z_{1}^{k}\right)_{k}$ is a complete preference sequence, there exists a $k^{*}(j) \in \mathbb{N}$, such that

$$
e_{i} e_{j} \succeq_{1}^{k} \tilde{p}_{c_{j}}
$$

for all $k \geq k^{*}(j)$. Let $k^{*}:=\max \left\{k^{*}(j) \mid j \in . J\right\}$. Then (6.6) follows from the' expected utility theorem for all $k \geq k^{*}$. This completes the prouf.

The first part of the last lemma implies $|I| \leq|. J|$, whereas the second part implies the opposite.

Corollary 6.29 Let $I \subseteq M I . J \subseteq N$ and let $\left(\succeq_{1}^{k}, \succeq_{2}^{k}\right)_{k \in N}$ be a two-persone complete preference sequence. Furthermore, let $\left(p^{k}, q^{k}\right)$ be' a sequen's of $\left(-z_{1}^{k},-z_{2}^{k}\right)$ equilibria such that $I=\operatorname{supp}\left(p^{k}\right)$ and $J=\operatorname{supp}\left(q^{k}\right)$ for all $k \in \mathbb{N}$. Then $|I|=|. I|$.

The next Lemma is a generalization of the corresponding result in Chapter 4.

Lemma 6.30 Let $I \subseteq M . J \subseteq N$ and let $\left(\beth_{1}^{k}, \beth_{2}^{k}\right)_{k \in \mathbb{N}}$ be a two-persone complete preference sequence. Furthermore, let $\left(p^{k} \cdot q^{k}\right)$ br a sequence of $\left(\succeq_{1}^{k} \cdot \succeq_{2}^{k}\right)$ equilibria such that $I=\operatorname{supp}\left(p^{k}\right)$ and $I=\sup n\left(q^{k}\right)$ for all $k \in \mathbb{R}$. Let $i \in I$ and $\hat{\jmath} \in J$ such that

$$
\begin{aligned}
& \min \{\tau(i, j) \mid j \in J\}<\min \{\tau(\hat{\imath}, j) \mid j \in J\} \text { for all } i \in I \backslash\{\hat{\imath}\} \text { and } \\
& \min \{\sigma(i, j) \mid j \in J\}<\min \{\sigma(i, j) \mid j \in J\} \text { for all } j \in J \backslash\{\hat{j}\} .
\end{aligned}
$$

Then (i) $\lim _{k \rightarrow \infty} p_{i}^{k}=1$ and (ii) $\lim _{k \rightarrow \infty} q_{j}^{k}=1$.

Proof. We prove (ii); the proof of (i) is analogous. By Lemma 6.28 and Corollary 6.29 we may renumber the strategies of the players such that

(a) $I=J=\{1, \ldots, s\}$ for some $s \geq 1$;

(b) $\sigma(j, j)<\sigma(i, j)$ for every $j \in J$ and $i \in I \backslash\{j\}$;

(c) $\sigma(1,1)<\sigma(2,2)<\ldots<\sigma(s, s)$.

Note that $\hat{\jmath}=s$. Let $s^{\prime} \in\{1, \ldots, s-1\}$ be arbitrary. To prove. (ii) it is sufficient to prove that $\lim _{k \rightarrow \infty} q_{s^{\prime}}^{k}=0$. Suppose that this is not the case. Then there is a $\alpha>0$ such that $q_{s^{\prime}}^{k} \geq \alpha$ for all $k$ (otherwise there is a subsecquence with this property). We define the following strategy

$$
\hat{p}:=\left\{\begin{array}{cc}
\frac{1}{s^{\prime}} & i \leq s^{\prime} \\
0 & \text { otherwise }
\end{array}\right.
$$

and prove that $e^{s^{\prime}+1} q^{k} \succeq_{1}^{k} \hat{p} q^{k}$, which contradicts Lemma 6.24 .

It is easily seen that $\hat{p}_{1}=\frac{1}{s^{\prime}}$ and $\sigma(1,1)<\sigma(i, j)$ for all $(i, j) \neq(1,1)$. Hence, the proof is completed by Bad Outcome Aversion.

Consider the following converse of Lemma 6.28 , 
工emma 6.31 Let $I \subseteq M$ and $J \subseteq N$ satisfy conditions (i) and (ii) in Lemma G. 28 and let $\left(\succeq_{1}^{k}, \succeq_{2}^{k}\right)_{k \in \mathbb{N}}$ be a two-persone complete preference sequence. Then for every $k \in \mathbb{N}$ there are $p^{k} \in \Delta(M)$ and $q^{k} \in \Delta(N)$ with supp $\left(p^{k}\right)=I$ and supp $\left(q^{k}\right)=J$ such that $\left(p^{k}, q^{k}\right)$ is a $\left(\succeq_{1}^{k}, \succeq_{2}^{k}\right)$-equilibrium.

Proof. First consider the case $|I|=|J|=1$. Then for every $k \in \mathbb{N}$ the strategy pair $\left(p^{k}, q^{k}\right)$ with $\operatorname{supp}\left(p^{k}\right)=I, \operatorname{supp}\left(q^{k}\right)=J$ is a pure Nash equilibrium with respect to every $u^{k} \in U^{k}$. Therefore we may consider the case $|I|=|J| \geq 2$. Again we may renumber the strategies of both players such that

(a) $I=J=\{1, \ldots, s\}$ for some $s \geq 1$;

(b) $\sigma(j, j)<\sigma(i, j)$ for every $j \in J$ and $i \in I \backslash\{j\}$;

(c) $\sigma(1,1)<\sigma(2,2)<\ldots<\sigma(s, s)$.

Take a fixed $k \in \mathbb{N}$ and define a strategy $q^{k} \in \Delta(N)$ with $\operatorname{supp}(q)=\{1, \ldots, s\}$ such that

$$
\frac{q_{j-1}^{k}}{q_{j}^{k}}:=\frac{1}{(m n)^{3}}\left|\frac{\psi(k)}{\xi(k)}\right| \text { for every } j=2, \ldots, s,
$$

where,

$$
\begin{aligned}
& \xi(k):=\max _{\substack{\left(i^{\prime}, j^{\prime}\right),\left(i^{\prime \prime}, j^{\prime \prime}\right) \in M \times N \\
u^{k} \in U^{k}}}\left(u_{\sigma\left(i^{\prime}, j^{\prime}\right)}^{k}-u_{\sigma\left(i^{\prime \prime}, j^{\prime \prime}\right)}^{k}\right) \\
& \psi(k):=\min _{\substack{\left(i^{\prime}, j^{\prime}\right),\left(i^{\prime \prime}, j^{\prime \prime}\right) \in M \times N \\
u^{k} \in U^{k}}}\left(u_{\sigma\left(i^{\prime}, j^{\prime}\right)}^{k}-u_{\sigma\left(i^{\prime \prime}, j^{\prime \prime}\right)}^{k}\right)
\end{aligned}
$$

We will show that every $p \in \Delta(M)$ with $\operatorname{supp}(p) \subseteq I$ is a $\succeq_{1}^{k}$-best reply against $\boldsymbol{q}^{\boldsymbol{k}}$. Since we can proof the analogous statement for player 2 , the proof is complete.

So let $p \in \Delta(M)$ with $\operatorname{supp}(p) \subseteq I$. Assume that $p$ is not a $\succeq_{1}^{k}$-best reply against $q^{k}$. Then there exists a $p^{\prime} \in \Delta(M)$ such that $p^{\prime} q^{k} \succeq_{1}^{k} p q^{k}$, which is ecruivalent to

$$
\sum_{i \in \operatorname{supp}\left(p^{\prime}\right)} \sum_{j \in J} p_{i}^{\prime} q_{j}^{k} u_{\sigma(i, j)}^{k} \geq \sum_{i \in \operatorname{supp}(p)} \sum_{j \in J} p_{i} q_{j}^{k} u_{\sigma(i, j)}^{k}
$$

for every $u^{k} \in U^{k}$. We can assume w.l.o.g. that $\operatorname{supp}(p) \cap \operatorname{supp}\left(p^{\prime}\right)=\varnothing$. Namely, suppose that this intersection is nonempty. Let $i$ be an element of the intersection and let $\alpha:=\min \left\{p_{i}, p_{i}^{\prime}\right\}$. Define

xxel

$$
\bar{p}:=\frac{1}{1-\alpha}\left(p-\alpha e^{i}\right)
$$

$$
\ddot{p}^{\prime}:=\frac{1}{1-\alpha}\left(p^{\prime}-\alpha e^{i}\right)
$$


Then it still holds that $\bar{p}^{\prime} q^{k} \succeq_{1}^{k} \bar{p} q^{k}$, whereas $i \notin \operatorname{supp}(\bar{p}) \cap \operatorname{supp}\left(\bar{p}^{\prime}\right)$.

So assume that $\operatorname{supp}(p) \cap \operatorname{supp}\left(p^{\prime}\right)=\oslash$ and take an $\hat{\imath} \in \operatorname{supp}\left(p^{\prime}\right)$ such that $p_{i}^{\prime} \geq \frac{1}{m}$. If $\hat{\imath} \in I$ then let $\hat{\jmath}:=\hat{\imath}$. Then by condition $(b), \sigma(\hat{\imath}, \hat{\jmath})<\sigma(i, \hat{\jmath})$ for all $i \in I \backslash\{\hat{\imath}\}$, and thus for all $i \in \operatorname{supp}(p)$ in particular. If $\hat{\imath} \in M \backslash I$ then by condition $(i)$ in Lemma 6.28 we can take $\hat{\jmath} \in J$ such that $\sigma(\hat{\imath}, \hat{\jmath})<\sigma(i, \hat{\jmath})$ for all $i \in I$ and therefore for all $i \in \operatorname{supp}(p)$. Together with conditions $(b)$ and $(c)$ this implies $\sigma(\hat{\imath}, \hat{\jmath})<\sigma(i, j)$ for all $i \in \operatorname{supp}(p)$ and $j \in\{\hat{\jmath}, \ldots, s\}$.

By Theorem 6.14 there is a sequence $\left(u^{k}\right)_{k \in \mathbb{N}}, u^{k} \in U$ and $u_{\sigma(\hat{i}, j)}^{k}<u_{n}^{k}$ for each $k \in \mathbb{N}$, such that

$$
\lim _{k \rightarrow \infty} \frac{u_{m n}^{k}-u_{\sigma(i, j)+1}^{k}}{u_{m n}^{k}-u_{\sigma(\hat{i}, j)}^{k}}=0
$$

We now evaluate

$$
\begin{aligned}
\mathbb{E}_{u^{k}}\left(p^{\prime}\right)-\mathbb{E}_{u^{k}}(p):= & \sum_{i \in \operatorname{supp}\left(p^{\prime}\right)} \sum_{j \in J} p_{i}^{\prime} q_{j}^{k} u_{\sigma(i, j)}^{k}-\sum_{i \in \operatorname{supp}(p)} \sum_{j \in J} p_{i} q_{j}^{k} u_{\sigma(i, j)}^{k} \\
= & \sum_{j \in J} q_{j}^{k}\left(\sum_{i \in \operatorname{supp}\left(p^{\prime}\right)} p_{i}^{\prime} u_{\sigma(i, j)}^{k}-\sum_{i \in \operatorname{supp}(p)} p_{i} u_{\sigma(i, j)}^{k}\right) \\
= & \sum_{j \leq \hat{j}} q_{j}^{k}\left(\sum_{i \in \operatorname{supp}\left(p^{\prime}\right)} p_{i}^{\prime} u_{\sigma(i, j)}^{k}-\sum_{i \in \operatorname{supp}(p)} p_{i} u_{\sigma(i, j)}^{k}\right) \\
& +\sum_{j>\hat{j}} q_{j}^{k}\left(\sum_{i \in \operatorname{supp}\left(p^{\prime}\right)} p_{i}^{\prime} u_{\sigma(i, j)}^{k}-\sum_{i \in \operatorname{supp}(p)} p_{i} u_{\sigma(i, j)}^{k}\right)
\end{aligned}
$$

Dividing by $q_{j}^{k}$ and rewriting yields

$$
\begin{aligned}
\frac{\mathbb{E}_{u^{k}}\left(p^{\prime}\right)-\mathbb{E}_{u^{k}}(p)}{q_{j}^{k}}= & \sum_{j<j} \frac{q_{j}^{k}}{q_{j}^{k}}\left(\sum_{i \in \operatorname{supp}\left(p^{\prime}\right)} p_{i}^{\prime} u_{\sigma(i, j)}^{k}-\sum_{i \in \operatorname{supp}(p)} p_{i} u_{\sigma(i, j)}^{k}\right) \\
& +\sum_{i \in \operatorname{supp}\left(p^{\prime}\right)} p_{i}^{\prime} u_{\sigma(i, j)}^{k}-\sum_{i \in \operatorname{supp}(p)} p_{i} u_{\sigma(i, j)}^{k} . \\
& +\sum_{j>\hat{j}} \frac{q_{j}^{k}}{q_{\hat{j}}^{k}}\left(\sum_{i \in \operatorname{supp}\left(p^{\prime}\right)} p_{i}^{\prime} u_{\sigma(i, j)}^{k}-\sum_{i \in \operatorname{supp}(p)} p_{i} u_{\sigma(i, j)}^{k}\right)
\end{aligned}
$$

By construction we have

$$
\lim _{k \rightarrow \infty} \sum_{i \in \operatorname{supp}(p)} p_{i} u_{\sigma(i, \hat{j})}^{k}=\lim _{k \rightarrow \infty} u_{\sigma(\hat{i}, \hat{j})+1}^{k}
$$


and

$$
\begin{aligned}
\lim _{k \rightarrow \infty} \sum_{i \in \operatorname{supp}\left(p^{\prime}\right)} p_{i}^{\prime} u_{\sigma(i, j)}^{k}= & \lim _{k \rightarrow \infty}\left[\left(1-p_{\hat{\imath}}\right) u_{\sigma(\hat{\imath}, \hat{\jmath})+1}^{k}+p_{\hat{\imath}} u_{\sigma(\hat{\imath}, \hat{\jmath})+1}^{k}\right. \\
& \left.-p_{i}\left(u_{\sigma(\hat{\imath}, \hat{\jmath})+1}^{k}-u_{\sigma(\hat{\imath}, \hat{\jmath})}^{k}\right)\right] \\
= & \lim _{k \rightarrow \infty}\left[u_{\sigma(\hat{\imath}, \hat{\jmath})+1}^{k}-p_{\hat{\imath}}\left(u_{\sigma(\hat{i}, \hat{\jmath})+1}^{k}-u_{\sigma(\hat{\imath}, \hat{\jmath}}^{k}\right)\right] \\
\leq & \lim _{k \rightarrow \infty}\left[u_{\sigma(\hat{\imath}, \hat{\jmath})+1}^{k}-\frac{1}{m}\left(u_{\sigma(\hat{\imath}, \hat{\jmath})+1}^{k}-u_{\sigma(\hat{\imath}, \hat{\jmath})}^{k}\right)\right] .
\end{aligned}
$$

This implies for $k$ large enough

$$
\begin{aligned}
& \frac{\mathbb{E}_{u^{k}}\left(p^{\prime}\right)-\mathbb{E}_{u^{k}}(p)}{q_{j}^{k}} \\
\leq & \sum_{j<j} \frac{q_{j}^{k}}{q_{j}^{k}}\left(\sum_{i \in \operatorname{supp}\left(p^{\prime}\right)} p_{i}^{\prime} u_{\sigma(i, j)}^{k}-\sum_{i \in \operatorname{supp}(p)} p_{i} u_{\sigma(i, j)}^{k}\right)-\frac{1}{m}\left(u_{\sigma(\hat{i}, \hat{\jmath})+1}^{k}-u_{\sigma(\hat{\imath}, \hat{\jmath})}^{k}\right) \\
& +\sum_{j>\hat{j}} \frac{q_{j}^{k}}{q_{\hat{\jmath}}^{k}}\left(\sum_{i \in \operatorname{supp}\left(p^{\prime}\right)} p_{i}^{\prime} u_{\sigma(i, j)}^{k}-\sum_{i \in \operatorname{supp}(p)} p_{i} u_{\sigma(i, j)}^{k}\right) \\
\leq & (m n)^{2} \cdot\left(\frac{q_{1}^{k}}{q_{2}^{k}}\right) \cdot \max _{\left.\left(i^{\prime}, j^{\prime}\right), i^{\prime \prime}, j^{\prime}\right) \in M \times N}\left(u_{\sigma\left(i^{\prime}, j^{\prime}\right)}^{k}-u_{\sigma\left(i^{\prime \prime}, j^{\prime \prime}\right)}^{k}\right) \\
& -\frac{1}{m}\left(u_{\sigma(\hat{i}, \hat{j})+1}^{k}-u_{\sigma(\hat{i}, \hat{\jmath})}^{k}\right) \\
< & 0
\end{aligned}
$$

This, however, is a contradiction to our assumption, which completes the proof.

The previous lemmata can be summarized as follows.

Theorem 6.32 Let $\left(\succeq_{1}^{k}, \succeq_{2}^{k}\right)_{k \in \mathbb{N}}$ be a two-persone complete preference sequence. If $I \subseteq M, J \subseteq N$ and $\left(p^{k}, q^{k}\right)_{k \in \mathbb{N}}$ is a sequence of $\left(\succeq_{1}^{k}, \succeq_{2}^{k}\right)$-equilibria such that $\operatorname{supp}\left(p^{k}\right)=I$ and $\operatorname{supp}\left(q^{k}\right)=J$ for all $k \in \mathbb{N}$ then (i) and (ii) in Lemma 6.28 hold,$|I|=|J|$, and the sequence of $\left(\succeq_{1}^{k}, \succeq_{2}^{k}\right)$-equilibria converges to the pure strategy combination $(\hat{\imath}, \hat{\jmath})$, where $\hat{\imath}$ and $\hat{\jmath}$ are as in Lemma 6.28. Conversely, if $\oslash \neq I \subseteq M$ and $\oslash \neq J \subseteq N$ satisfy (i) and (ii) in Lemma 6.28, then a sequence of $\left(\succeq_{1}^{k}, \succeq_{2}^{k}\right)$-equilibria with supports $I$ and $J$ for players 1 and 2 , respectively, exists. Furthermore, let $\left(p^{k}, q^{k}\right) \in \Delta(I) \times \Delta(J)$ be a sequence such that $I=\operatorname{supp}\left(p^{k}\right)$ and $J=\operatorname{supp}\left(q^{k}\right)$ for all $k \in \mathbb{N}$.

\section{Appendix: proof of Lemma 6.9}

Lemma 6.33 For all $t, t^{\prime} \in \mathbb{R}, t, t^{\prime} \geq 1$, we have

$$
A_{t+t^{\prime}}=A_{t} A_{t^{\prime}} .
$$


Proof. Let $i, j \in\{1 \ldots \ldots\}$ and $j \geq 1$. Wh have to show

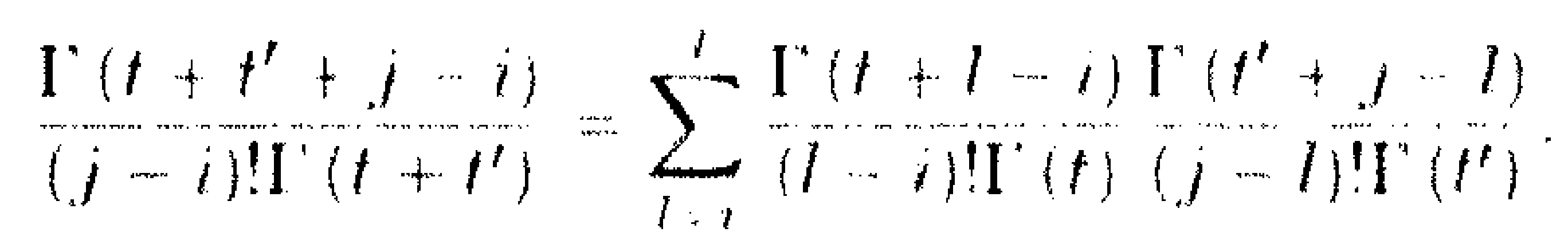

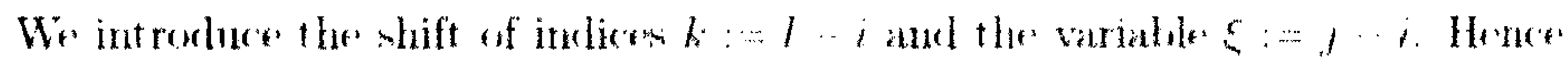
wo have to show

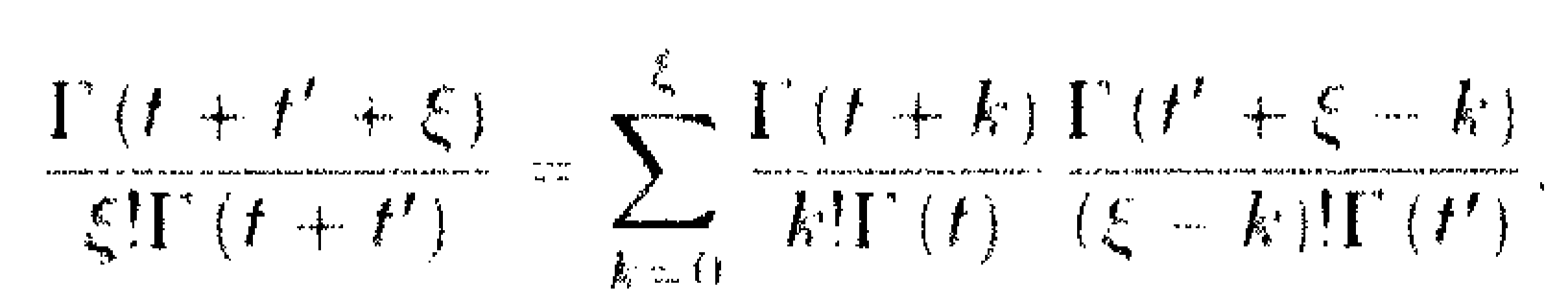

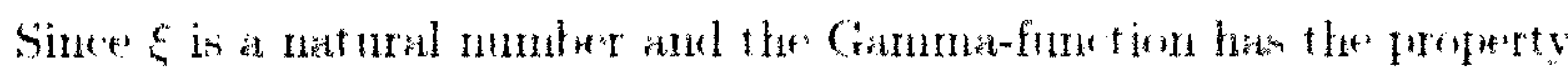

$$
x \Gamma(x)=\Gamma(x+1)
$$

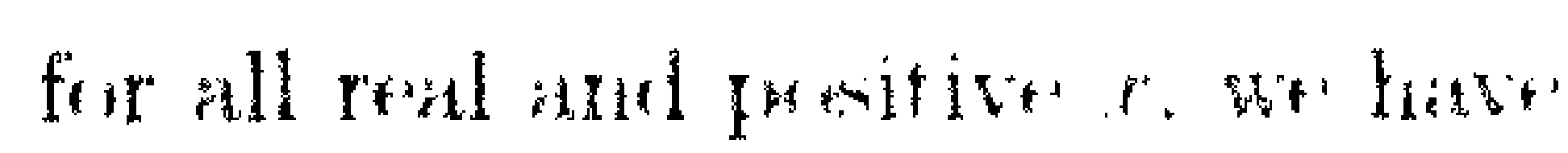

$$
\left[\begin{array}{l}
1(r+s) \\
\Gamma(r)
\end{array}=(r+s-1)(x+s-2) \ldots r .\right.
$$

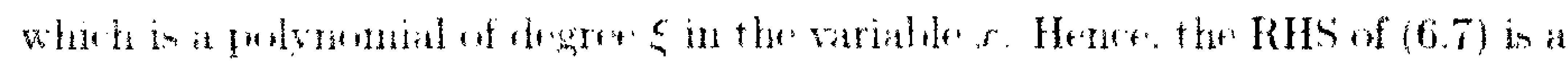

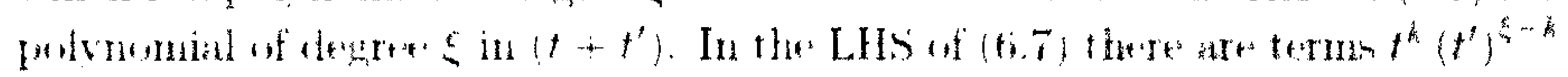

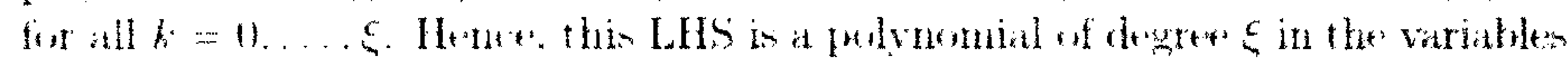

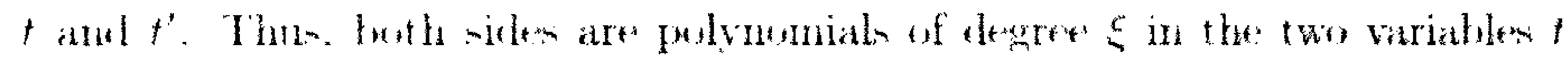
and $t^{\prime}$. Furthermore. from the representation for natural tas given in Clatpter

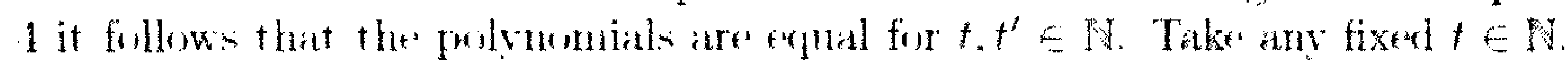

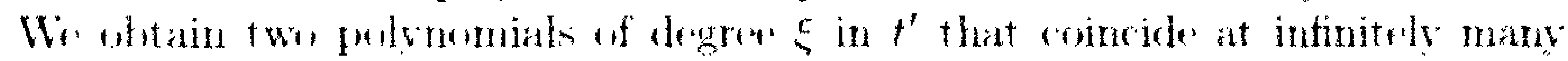
points (all natural $t^{\prime}$ ) and hence. these polymmials are orpal for all $t^{\prime} \in \mathbb{F}^{2}$ $t \geq 0$. Next. We take any $t^{\prime} \in \mathbb{R} . t^{\prime} \geq 0$ and obtain two polynumak of degree

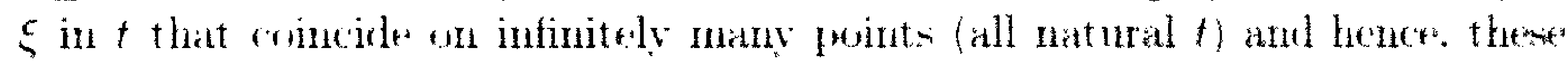

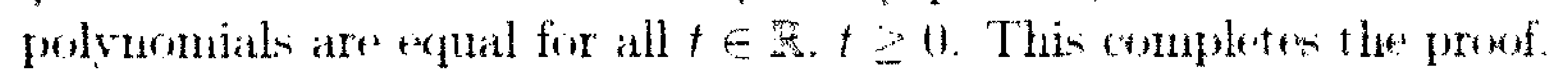




\section{Bibliography}

[Aumann, 1974]

[Aumann, 1962]

[Banks \& Sobel. 1987]

[Battigalli. 2003]

[Börgers, 1993]

[Borm et al. 2003]

[Cho \& Kreps, 1987]

[Dubra et al, 2004]

[Fishburn, 1976]

[Fishburn, 1978]
R.J. Aumann (1974): "Subjectivity and Correlation in Randomized Strategies", Journal of Mathematical Economics 1:67-96.

R.J. Aumann (1962): "Utility theory without the completeness axiom", Econometrica 30(1962): 445462.

J. Banks ans J. Sobel, Equilibrium selection in signaling games, Econometrica. 55(1987), 647-661.

P. Battigalli (2003): "Rationalizability in infinite. dynamic games with incomplete information", Research in Economics 57 (2003), 1-38.

Pure strategy dominance, Econometrica 61: 423-430.

Peter Borm, Dries Vermeulen, Mark Voomeveld. The structure of the set of equilibria for two person multicriteria games; European Journal of Operational Research 148 (2003) 480-493.

I.-K. Cho and D.M. Kreps, Signaling games and stable equilibria, Quarterly Journal of Economics 102(1987), 179-221.

Dubra J, Maccheroni F, Ok E (2004) Expected utility theory without the completeness axiom. Jomrnal of Economic Theory 115:118-133.

Fishburn PC (1976) Continua of stochastic dominance relations for bounded probability distributions. Journal of Mathematical Economics 3:29\% 311.

Fishburn PC (1978) Non-cooperative stochastir dominance ganes. International Journal of Gant Theory 7:51-61. 
[Fishburn, 1980]

P.C. Fishburn (1980): "Stochastic Dominance and Moments of Distributions", Mathematics of Operations Research, 5, 94-100.

[Grossman \& Perry, 1986] S. Grossman and M. Perry, Perfect sequential equilibria, Journal of economic theory 39(1986), 97-119.

[Harsanyi, 1967]

J. Harsanyi, Games with incomplete information played by "Bayesian" players I-III, Management Science $14(1967,1968), 159-182,320-334,486-502$.

[Kreps \& Wilson, 1982] D. Kreps and R. Wilson, Sequential equilibria, Econometrica 50(1982), 863-894.

[Levy, 1992]

Levy H (1992) Stochastic dominance and expected utility: survey and analysis. Management Science 38:555-593.

[Nash, 1950]

J.F. Nash, Equilibrium points in n-person gamos. Proceedings from the National Academy of Science, USA, 36(1950), 48-49.

$[$ Nash, 1951]

J.F. Nash, Noncooperative games. Annals of Mathematics 54(1951), 286-295.

[Perea, 2003]

Andrés Perea, Forward Induction and the Minimum Revision Principle; revised version november 2003.

[Perea et al. 2006]

Perca A, Peters H, Schulteis T, Vermeulen D (2006) Stochastic dominance equilibria in two-person noncooperative games. International Journal of Game Theory 34(2006), 457-473.

[Rockafellar, 1970] R.T. Rockafellar, Convex Analysis, Princeton university Press 1970.

[Rothe, 1995]

Equilibrium selection in ordinal games. Mimeo, London school of Economics.

[Schulteis, 2007]

T. Schulteis, A. Perea, H.Peters and D. Vermeulen, Revision of conjectures about the opponent's utilities in signaling games, Economic Theory (2007) 30:373384.

[Shapley, 1959]

Equilibrium points in games with vector payoffs, Nav. Res. Logist Q 6:57-61.

[Tan \& Werlang, 1988] T. Tan and S.R.C. Werlang (1988): "The Baycsian foundations of solution concepts of games", Journal of Economic Theory 1988(45), 370-391. 


\section{Chapter 7}

\section{Nederlandse samenvatting (summary in dutch)}

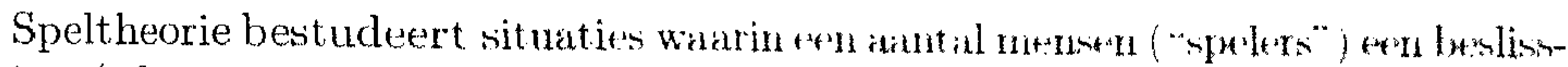

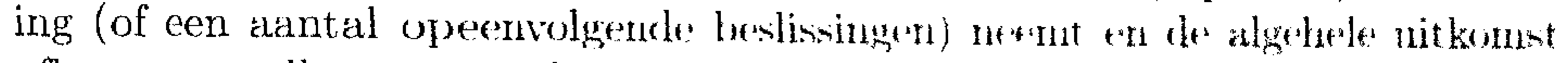
afhangt van alle genomen beslissingen. In dit fme tew thrift ben ik databij slechts

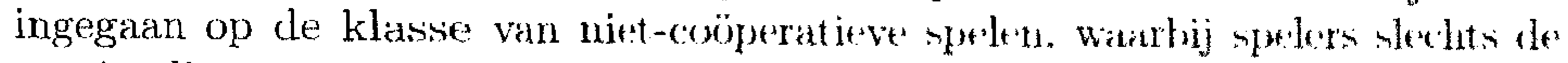
maximalisatie van hun eigen nut nastreven en gowen conlition vornten. In deel I ga ik vooral in op enkele anpecten dir te maken heblen met "lowliefs". i.e. de inschattingen die spelers maken m.b.t. zaken watarowtr zij in onzekerheid verkeren, zoals de strategiekenze' van hon t tegenstanders, de inschattingen die de tegenstanders van de strategieketuze van hü tegenstanders makt'n of. zwals we ook in deel I hebben gezien. de nutsfunct if van de tegenstanders.

In hoofdstuk 2 stel ik me de vraag wat speler 2 in extn "signialing ganie" moet denken en doen als hij een beslissing vam speler 1 wadrutent die hij. gegeven zijn inschatting van de situatie, als niet optinatal (en dus irrationetel) veor sperter 1 had ingeschat. Bekende evenwichtsconeepten zoals segnentieel evenwirht hientern geen mogelijkheid tot het reviseren van ean inschat ting. dus in dezt nodellern zon speler 2 de beslissing van zijn tegenstander als irrationed beschonwen fan blijven beschouwen. Ik houd daarentegen vast atn de atmome dat iredere sipeler rattioned is en denkt dat zijn tegenstander rationecel is. Daartoe dient sipele 2 zijn inschatting ten aanzien van grootheden op basis watarvan ineler 1 enth besliswing

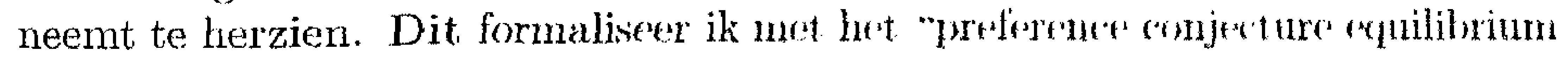

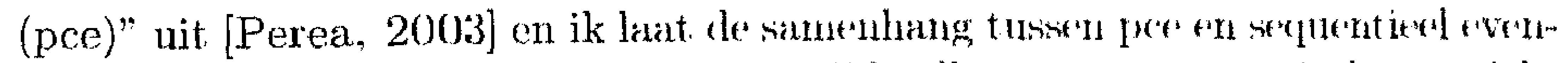

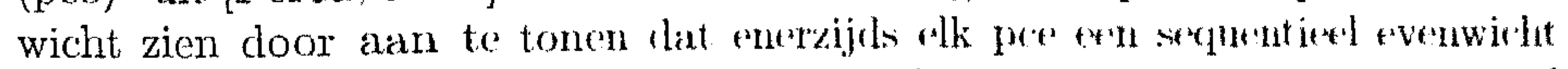

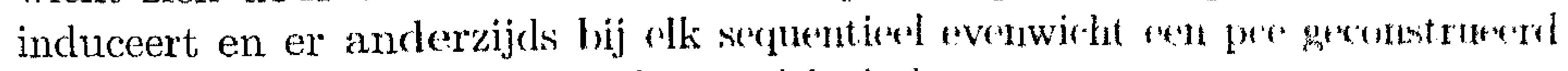
kan worden dat dit sequentieel avenwidht indurontrt.

Naturlijk zijn er vele manieren om de inschattingen zordanig to herzir'n dat de beslissing van speler 1 gerationaliseterd woralt. Daarom voer ik at'm matat in

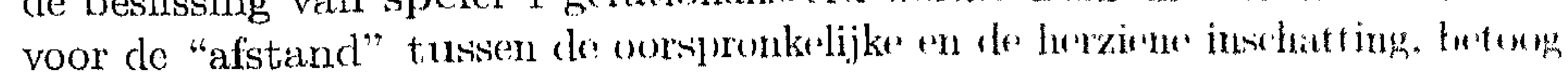


dat speler 2 kiest voor de kleinste herziening van zijn inschatting die in het kader van dit afstandsbegrip nodig is om de beslissing van zijn tegenstander te rationaliseren en onderzock de consequenties hiervan. Dit leidt tot een nieuw concept, namelijk "mininum revision equilibrium". Ook ga ik in op de eventuele samenhang van dit nieuwe concept met bestaande concepten.

In hoofdstuk 3 gebruik ik een epistemisch model om een bekend en belangrijk resultaat uit de literatuur te veralgemeniseren. In een epistemisch model wordt, cle structuur van iteratieve beliefs geformaliseerd. Stel twee spelers, A en B, spelen een spel. Voordat A zijn beslissing neemt, vormt hij een belief over wat hij denkt dat $B$ gaat doen. $B$ doet echter het zelfde, dus baseert A zijn keuze indirect eigenlijk ook op wat B denkt dat hij gaat doen. Ook dit geldt voor B, dus eigenlijk baseert $A$ zijn keuze ook op zijn inschatting van wat $B$ clenkt dat $A$ denkt dat $B$ gaat doen enzovoort. In concreto wordt in ons epistemisch model aangenomen dat A en B rationeel zijn, van elkatar denken dat ze rationeel zijn, van elkaar denken dat ze denken dat hun tegenstander rationeel is enzovoort. We formuleren dit model voor een spel met in het algemeen $n$ spelers.

In de litcratuur bestaat er een belangrijk resultaat [Tan \& Werlang, 1988] dat het proces van iteratieve eliminatie van strikt gedomineerde strategicc̈n verbindt met het principe "common belief in rationality". Het eliminatieproces voor strikt gedomineerde strategieën heb ik in de inleiding al met een cenvoudig voorbeeld toegelicht. Het is het proces waarbij de spelers eerst cigen purc strategieën "wegstrepen" die strikt gedomineerd zijn (waarvoor er dus een strategie bestaat, die, ongeacht de actie van de tegenstanders, beter is) en vervolgens aannemen dat hun tegenstanders dat ook doen. Vervolgens ontstaat er een 'gereduceerd spel' (qua aantal pure strategieën), waarin dit proces zich herhaalt (een pure strategie die in eerste instantie niet strikt gedomineerd was, kan dat nu wel zijn omdat er ook pure strategieën van de tegenstanders zijn "weggestreept"). Dit proces wordt herhaald tot er geen pure strategicën meer kunnen worden weggestreept. Met "common belief in rationality" bedoelen we het geloof ('belief') in de rationaliteit van de tegenstanders volgens het in de vorige alinea beschreven iteratieve proces. Het resultaat van [Tan \& Werlang, 1988] verbindt deze twee concepten door aan te tonen dat een pure strategie dan en slechts dan op rationele wijze gekozen kan worden onder "common belief in rationality" als deze strategie het iteratief eliminatieproces van strikt gedomineerde strategieën overleeft.

Het resultaat van [Tan \& Werlang, 1988] gaat ervan uit dat de nutsfuncties van alle spelers algemeen bekend zijn. Wij laten zien dat een soortgelijk resultaat ook bewezen kan worden als spelers onzekerheid hebben over elkaars nutsfuncties, mits we additionele eisen stellen aan de samengcstelde structuur van de klasse van nutsfuncties die spelers voor hun tegenstanders mogelijk achten en de strategiekeuzen van de spelers. Als voorbeeld passen we het hoofdresultaat toe op het bekende Cournot model, waaraan we onzekerheid over de marginale kostcn van de tegenstander hebben toegevoegd. Ons hoofdresultaat zou de basis voor een elegant en intuitief bewijs voor de stelling over "pure strategy dominance" van [Börgers, 1993] vormen, als we zouden kunnen bewijzen dat de 
daarbij bijbehorende klasse van monotone nutsfuncties in combinatie met de mogelijke strategiekeuzen aan de additionele eis voor toepasbaarheid van onze stelling voldoet. Dat laatste is ook gedurende de laatste tijd voor ons een open probleem gebleven (technisch gezien is de geslotenheid onder Harsanyi aggregatie respectievelijk de geslotenheid onder additie van betreffende verzamelingen te bewijzen).

In hoofdstuk 4 pas ik het principe van stochastische dominantie toe op tweepersoonsspelen. Stochastische dominantie is een manier om kansverdelingen over pure uitkomsten met elkaar te vergelijken als we slechts uitgaan van de aanname dat spelers een volledige, transitieve en asymmetrische preferentierelatie hebben over pure uitkomsten. Belangrijk is dat stochastische dominantie leidt tot onvolledige preferentierelaties over kansverdelingen. We verbinden onze analyse aan een recent paper van [Dubra et al, 2004], waarin de consequenties worden onderzocht van het laten vallen van de volledigheidsaanname uit de von Neumann Morgenstern condities. In [Dubra et al, 2004] wordt aangetoond dat er ook voor dit type preferentierelaties een representatiestelling bewezen kan worden. In hoofdstuk 6 ga ik daar nader op in. In hoofdstuk 6 laat ik ook zien dat de op het eerste gezicht tamelijk willekeurige manier om kansverdelingen te beoordelen met het criterium van stochastische dominantie voor een bepaalde klasse van onvolledige preferenticrelaties tot conclusies leidt die algemener waar zijn dan slechts voor stochastische dominantie (namelijk voor die hele klasse van onvolledige preferentierelaties).

Eerste graad stochastische dominantic leidt tot de conclusie dat een kansverdeling beter wordt door gewicht te verschuiven naar betere pure uitkomsten. Hoe hoger de graad van stochastische dominantie, hoe sterker dit effect. We zeggen dat een strategie een "beste antwoord" is als er geen andere strategie bestaat die een kansverdeling induceert die in termen van stochastische dominatie boven deze eerste strategie wordt geprefereerd. Aangezien de relatie onvolledig is, kunnen er paren van kansverdelingen zijn die niet met elkaar vergeleken kunnen worden. Een evenwicht construeren we vervolgens als paar van beste antwoorden. in de literatuur heeft Fishburn een verband gelegd tussen de verzamelingen evenwichten in termen van eerste orde stochastische dominantie en de verzameling Nash evenwichten van alle representerende nutsfuncties. Het gaat hierbij om een intuitief resultaat dat zegt dat een kansverdeling in termen van eerste orde stochastische dominantie ongedomineerd is als er tenminste één representerende nutsfunctie te vinden is waarvoor deze verdeling een beste antwoord is met betrekking tot die nutsfunctie.

In hoofdstuk 4 laat ik zien dat algemeen voor stochastische dominantie van orde $t$ een soortgelijk resultaat bewezen kan worden. Ook ga ik in op de consequenties van het toenemen van de graad van stochastische dominantie. Het blijkt dat de geïnduceerde preferentierelatie steeds minder onvolledig wordt (in de limiet zelfs volledig) en dat toenemende graad van stochastische dominantie een toenemende aversie tegen "slechte" uitkomsten implicecrt. Ook bewijs ik een limietresultaat waarin de verzameling van evenwichten wordt onderzocht voor de limiet waarin de graad van stochastische dominantic naar oneindig con- 
vergeert. Het resultaat laat zien dat voor bepaalde klassen van dragers (pure strategieën waaruit de spelers kiezen) een oncindige graad van stochastische dominantie impliceert dat spelers de strategie kiezen die voor hun tegenstander optimaal is (een vorm van altruïsme). Het resultaat impliceert ook dat in de limiet een lexicografische preferentierelatie ontstaat (en er dus maar één strategie overblijft als beste antwoord). Omgckeerd laat ik zien dat, omgekeerd, voor iedere drager die in deze klasse ligt een rijtje evenwichten met bovenstaande eigenschapen geconstrueerd kan worden.

Hoofdstuk 5 behandelt een in zekere zin natuurlijke uitbreiding van hoofdstuk 4. In niet coöperaticve spelen waarin de preferenties van de spelers door von Neumann - Morgenstern preferentierelaties zijn gegeven zijn er twee belangrijke evenwichtsconcepten: Nash evenwicht en gecorrelcerd evenwicht (zie ook de inleiding en hoofdstuk 5). Hoofdstuk 4 ging onder andere over de samenhang tussen Nash evenwich en $t$-evenwicht. Ik pas in hoofdstuk 5 de concepten uit hoofdstuk 4 toe op gecorrcleerd evenwicht, wat leidt tot het concept gecorreleerd t-evenwicht. Ook hier ga ik in op de limiet waarin de graad van stochastische dominantie naar oneindig convergeert. In tegenstelling tot hoofdstuk 4, waar in de limiet voor beide spelers een pure strategie resulteerde, kan hier ook een kansverdeling over strategieën als limiet bereikt worden. Ik laat zien dat, gegeven de drager van een limiet van gecorreleerde $t$-evenwichten, er voor iedere kansverdeling over deze support een rijtje gecorreleerde $t$-evenwichten geconstrueerd kan worden dat naar doze kansverdeling convergeert. Met andere woorden, als een bepaalde kanverdeling als limiet resulteert, kan ook ieder andere kansverdeling met dezelfde drager als limiet van gecorrelecrde t-evenwichten voorkomen.

In hoofdstuk 6 ga ik in op onvolledige preferentierelaties in algemenere 7 in. Allereerst ga ik in op de stelling uit [Dubra et al, 2004], de tegenhanger van de representatiestelling van Von Neumann en Morgenstern voor onvolledige preferentierelaties. Vervolgens geef ik een aantal axiomata, onafhankelijkheid (independence), "Improvement" en "Bad Outcome Aversion" (BOA). Volgens Improvement wordt een kansverdeling (zwak) geprefereerd boven een andere kansverdeling die slechts op twee punten van de eerste verschilt en op het slechtste van de twee pure alternatieven waarop ze verschillen meer gewicht legt. BOA is een algemene formulering van de eigenschap die we bij stochastische dominantie hadden gezien: een aversie tegen slechte uitkomsten. Ik laat zien dat en preferentierelatie die volledig is op pure uitkomsten en voldoet aan onafhankelijkheid daarmee een eerste orde stochastische dominantie relatie bevat. Verder geef ik ecn karakterisering in termen van BOA van preferentierelaties die volledig zijn op pure alternatieven en aan onafhankelijkheid en Improvement voldoen. Ik laat zien dat deze karakterisering zeer dicht tegen stochastische dominantie aanligt en bewijs voor de situatie van 3 pure toestanden dat er zelfs een equivalentie bestaat tussen de axiomata en stochastische dominantie voor een orde $t$. Daartoe breid ik het begrip $t$-de orde stochastische dominantie uit van natuurlijke $t$ naar reële waarden van $t$. 
In paragraaf 6.4 laat ik zien dat de belangrijkste resultaten uit hoofdstuk 4 algemeen gelden voor preferentierelaties die aan de axiomata voldoen. Zo blijft de karakterisering van evenwichten in termen van Nash evenwichten met betrckking tot representerende nutsfuncties overeind. Ook de limietstellingen voor de limiet naar oneindige aversie tegen slechte uitkomsten geldt algemeen. Daarmee is aangetoond dat stochastische dominantie weliswaar een zeer specifieke keuze is om onvolledige preferentierelaties te waarderen, maar op een aantal fundamentele punten tot dezelfde resultaten leidt als iedere andere preferentierelatir die aan de veel algemenere axiomata voldoet. 


\section{Curriculum vitae}

Tim Schulteis was born on July 31st 1979 in Kerkrade, where he attended high school at Katholiek Gymnasium Rolduc from 1990 untill 1996. In 1996 he started his studics of Mathematics and Physics at the Technical University RWTH Aachen in Germany, where he graduated in Physics at the department of Theoretical Physics in 2002 with a thesis on the use of the theory of stochastic processes in the numerical sinulation of reaction-diffusion-systems. As a counterpart of his technical education Tim became more and more interested in non-technical applications of mathematical models and in 2002 he became a part time Ph.D. student at Maastricht University under the supervision of dr.ir. Andrós Perea, prof. dr. Hans Peters and dr. Dries Vermeulen in the field of game theory, especially noncooperative game theory. He combined his research with the study of music, choral conducting and singing, at the Conservatorium Maastricht. In september 2007 he will finish his musical studies by earning the degree of Master in choral conducting. During the last years he has also been working as a conductor of several choirs and as freelance singer, both in choirs and as a soloist and he attended several master classes. Together with his wife Esther Zaad and some other enthousiastic colleagues Tim was one of the founders of "Vocaal Ensemble Virenze" (2004), a vocal ensemble consisting of 6 to 8 professional musicians that studies and performs music from all periods in musical history, from the middle ages to contemporary music, with a special predilection for the polyphonic masters of the Renaissance.

Subsequent to his work as a Ph.D. student at Maastricht University Tim Schulteis works as an actuary at the pension fund ABP in Heerlen. 